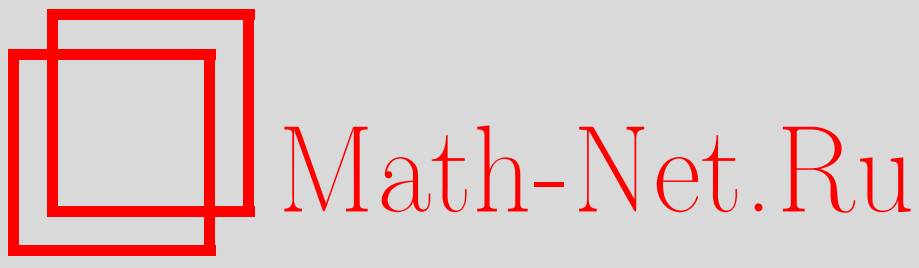

Ю. А. Алхутов, О. В. Крашенинникова, Непрерывность в граничных точках решений квазилинейных эллиптических уравнений с нестандартным условием роста, Изв. РАН. Сер. матем., 2004, том 68, выпуск 6, 3-60

DOI: https://doi.org/10.4213/im509

Использование Общероссийского математического портала Math-Net.Ru подразумевает, что вы прочитали и согласны с пользовательским соглашением

http: //www. mathnet.ru/rus/agreement

Параметры загрузки:

IP : 52.6 .47 .48

26 апреля 2023 г., 06:36:06 
УДК 517.946

Ю.А. Алхутов, О.В. Крашенинникова

\section{Непрерывность в граничных точках решений квазилинейных эллиптических уравнений с нестандартным условием роста}

\footnotetext{
Исследуется вопрос о поведении в граничных точках решения задачи Дирихле с непрерывной граничной функцией для уравнения Эйлера, порожденного интегрантом $|\nabla u|^{p(x)} / p(x)$ с переменным показателем суммируемости $p=p(x)$, обладающим логарифмическим модулем непрерывности и удовлетворяющим условию $1<p_{1} \leqslant p(x) \leqslant p_{2}<\infty$. Получен критерий регулярности граничной точки винеровского типа, найдена оценка модуля непрерывности решения вблизи регулярной граничной точки, приведены геометрические условия регулярности.

Библиография: 40 наименований.
}

\section{$\S 1$. Введение}

Рассмотрим в ограниченной области $D$ евклидова пространства $\mathbb{R}^{n}, \quad n \geqslant 2$, уравнение

$$
L u=\operatorname{div}\left(|\nabla u|^{p(x)-2} \nabla u\right)=0
$$

с измеримой в $D$ функцией $p(x)$, удовлетворяющей условию

$$
1<p_{1} \leqslant p(x) \leqslant p_{2}<\infty .
$$

Если $p(x)=$ const, то квазилинейное уравнение (1.1) и его естественные обобщения детально изучены. Обзор литературы по таким уравнениям содержится в монографии [1].

Уравнение (1.1) относится к обширному классу эллиптических уравнений с нестандартным условием роста и является уравнением Эйлера для вариационных задач с интегрантом $|\nabla u|^{p(x)} / p(x)$. Соответствующие вариационные задачи изучались в 1980-х годах В.В. Жиковым в [2]-[4]. В этих работах основное внимание было уделено эффекту Лаврентьева, о котором более подробно будет сказано ниже, и его связи с задачами усреднения. Кроме того, в работе [4] был приведен пример нерегулярного решения вариационной задачи, сыгравший важную роль в изучении качественных свойств решений уравнения (1.1). Этот пример и ряд других примеров разрывных и неограниченных решений вариационных задач, построенных М. Джиаквинтой в [5] и П. Марчеллини в [6], инициировали исследование свойств регулярности решений эллиптических уравнений с нестандартным условием роста. Последние десять лет данная тематика активно

Работа выполнена при финансовой поддержке РФФИ (проекты № 03-01-00138, 04-0100717) и Минобразования РФ (грант Е02-1.0-57).

(C) Ю.А. АлхУтов, О.В. КРАшенИнниковА, 2004 
развивается и имеет не только самостоятельньй интерес. Уравнение вида (1.1) и системы таких уравнений возникают во многих задачах математической физики, описание которых содержится в [7]. Одним из примеров является нелинейная система, описывающая динамику жидкостей, которые могут изменять свойства под действием электромагнитного поля. Другой пример - задача о термисторе (см. [8]). Система эллиптических краевых задач

$$
\begin{aligned}
& -\operatorname{div}\left(|\nabla u|^{p(x)-2} \nabla u\right)=f \quad \text { в } \quad D,\left.\quad u\right|_{\partial D}=0, \\
& -\Delta p=|\nabla u|^{p(x)} \quad \text { в } D,\left.\quad p\right|_{\partial D}=\text { const }>1,
\end{aligned}
$$

описывает взаимосвязь потенциала электрического поля $u(x)$ и температуры $p(x)$ в термисторе $D$.

Прежде чем описать известные нам свойства решений уравнения (1.1) и сформулировать полученные результаты, определим понятие решения. Для этого введем класс функций

$$
W(D)=\left\{u: u \in W_{1}^{1}(D),|\nabla u|^{p(x)} \in L_{1}(D)\right\},
$$

где $W_{1}^{1}(D)$ - соболевское пространство функций, суммируемых в $D$ вместе с обобшенными производными первого порядка. Сходимость в классе $W(D)$ определим следующим образом.

ОПРЕДЕЛЕНИЕ 1.1. Последовательность функций $u_{j} \in W(D)$ сходится в $W(D) \kappa$ функции $u \in W(D)$, если $u_{j} \rightarrow u$ в $L_{1}(D)$ и

$$
\lim _{j \rightarrow \infty} \int_{D}\left|\nabla u-\nabla u_{j}\right|^{p(x)} d x=0
$$

В классе $W(D)$ можно определить норму Орлича

$$
\|u\|_{W(D)}=\|u\|_{L_{1}(D)}+\inf \left\{\lambda: \lambda>0, \int_{D}|\nabla u|^{p(x)} \lambda^{-p(x)} d x \leqslant 1\right\} .
$$

Сходимость по этой норме эквивалентна введенной выше, но нам удобнее пользоваться определением 1.1. Введем еще класс $W_{0}(D)$ как замыкание в смысле указанной выше сходимости множества функций из $W(D)$ с компактным носителем в $D$. Ниже мы используем еще сходимость в $W_{0}(D)$ : последовательность функций $u_{j} \in W_{0}(D)$ сходится в $W_{0}(D)$ к функции $u \in W_{0}(D)$, если выполнено равенство (1.3). Ясно, что в этом случае требование сходимости $u_{j}$ в $L_{1}(D)$ излишне.

Теперь мы можем дать определение решения уравнения (1.1). Это будет одно из возможных понятий решения, называемого $W$-решением.

ОПРЕДЕЛЕНИЕ 1.2. Функция $u \in W(D)$ называется $W$-решением уравнения (1.1), если интегральное тождество

$$
\int_{D}|\nabla u|^{p(x)-2} \nabla u \cdot \nabla \psi d x=0
$$

выполнено для пробных функций $\psi \in W_{0}(D)$. 
Из результатов работы [4] следует, что одного только предположения (1.2) недостаточно для плотности гладких функций в классах $W(D)$ и $W_{0}(D)$. В связи c этим возникают функциональные классы, порожденные гладкими функциями:

$$
\begin{aligned}
H(D) & =\left\{u: u \in W(D), \exists u_{j} \in C^{\infty}(D) \cap W(D), u_{j} \rightarrow u \text { в } W(D)\right\} \\
H_{0}(D) & =\left\{u: u \in W(D), \exists u_{j} \in C_{0}^{\infty}(D), u_{j} \rightarrow u \text { в } W_{0}(D)\right\} .
\end{aligned}
$$

Другими словами, $H(D)$ есть замыкание в $W(D)$ множества $W(D) \cap C^{\infty}(D)$, а $H_{0}(D)$ - замыкание в $W_{0}(D)$ множества $C_{0}^{\infty}(D)$. Как хорошо известно, в случае постоянного показателя суммируемости $W(D)=H(D)$ и $W_{0}(D)=H_{0}(D)$ для любой области $D$.

Важное свойство уравнения (1.1) состоит в том, что оно может иметь решения из класса $H(D)$, для которых интегральное тождество (1.4) выполнено только на пробных функциях $\psi \in H_{0}(D)$.

ОПРЕДЕЛЕНИЕ 1.3. Функция $u \in H(D)$ называется $H$-решением уравнения (1.1), если интегральное тождество (1.4) выполнено на пробных функциях $\psi \in$ $H_{0}(D)$.

Введенные выше $W$-решения и $H$-уравнения (1.1) связаны с задачами Дирихле

$$
L u=0 \quad \text { в } D, \quad u \in W(D), \quad h \in W(D), \quad(u-h) \in W_{0}(D)
$$

и

$$
L u=0 \quad \text { в } D, \quad u \in H(D), \quad h \in H(D), \quad(u-h) \in H_{0}(D)
$$

соответственно.

Задачи Дирихле (1.5) и (1.6) были введены В. В. Жиковым в [3] под названием задач первого и второго рода. Он же заметил, что если коразмерность класса $H$ в $W$ больше единицы, то существуют и другие промежуточные задачи Дирихле и соответствуюшие им решения уравнения (1.1).

Доказательство разрешимости задачи Дирихле (1.5) основано на исследовании вариационной задачи

$$
\min _{w \in W_{0}(D)} F(w+h), \quad F(u)=\int_{D} \frac{|\nabla u|^{p(x)}}{p(x)} d x, \quad h \in W(D) .
$$

Если измеримьй в $D$ показатель суммируемости удовлетворяет только условию (1.2), то возможна ситуация, при которой

$$
\min _{w \in W_{0}(D)} F(w+h)<\inf _{w \in C_{0}^{\infty}(D)} F(w+h)
$$

даже в случае, когда область $D$ совпадает с шаром, а $h \in C^{\infty}(\bar{D})$. Такого рода неравенства принято называть эффектом Лаврентьева по имени ученого, обнаружившего подобное свойство в работе [9] на примере одномерной вариационной 
задачи. Для рассматриваемых нами функционалов эффект Лаврентьева исследован в работах [4], [8], [10]. Если для решения вариационной задачи (1.7) не существует минимизирующей последовательности из $C_{0}^{\infty}(D)$, то наряду с $(1.7)$ возникает задача

$$
\inf _{w \in C_{0}^{\infty}(D)} F(w+h)=\min _{w \in H_{0}(D)} F(w+h), \quad h \in H(D) .
$$

Для областей с липшицевой границей однозначная разрешимость задач (1.7), (1.8) при выполнении одного только условия (1.2), налагаемого на измеримьй показатель суммируемости $p(x)$, установлена в работах [8], [10]. Отметим, что если разрешимость задачи (1.7) следует из обших соображений выпуклого анализа, то с разрешимостью задачи (1.8) дело обстоит несколько иначе. Разрешимость этой задачи вытекает из [10, теорема 5.2], но доказательство этой теоремы неэлементарно, в частности, использует теорию двойственности. В теореме 3.1 предложено элементарное исследование вариационных задач $(1.7),(1.8)$ в произвольной ограниченной области $D$, основанное на доказательстве сходимости минимизирующей последовательности в классе $W_{0}(D)$.

Задачи Дирихле (1.5) и (1.6) также однозначно разрешимы (теорема 3.2). Доказательство основано на том (см. [10], [11]), что (1.1) есть уравнение Эйлера для вариационных задач $(1.7),(1.8)$ и минимизант $w(x)$ каждой из них связан с решением $u(x)$ соответствующей задачи Дирихле равенством $w(x)=u(x)-h(x)$.

В настояшей работе рассмотрена и задача Дирихле для неоднородного уравнения

$$
L u=-d \mu \text { в } D, \quad u \in H_{0}(D),
$$

с мерой $\mu$, удовлетворяющей условию

$$
\exists v \in W(D), \quad\left|\int_{D} \psi d \mu\right| \leqslant C \int_{D}|\nabla v|^{p(x)-1}|\nabla \psi| d x \quad \forall \psi \in C_{0}^{\infty}(D)
$$

Под решением понимается функция $u \in H_{0}(D)$, для которой

$$
\int_{D}|\nabla u|^{p(x)-2} \nabla u \cdot \nabla \psi d x=\int_{D} \psi d \mu \quad \forall \psi \in C_{0}^{\infty}(D) .
$$

Однозначная разрешимость задачи (1.9) доказана в теореме 3.3 , сушественно используемой при исследовании граничных свойств решений уравнения (1.1).

Отметим, что для уравнения (1.1) с постоянным показателем суммируемости $p(x)$ и естественных обобщений такого уравнения однозначная разрешимость задач Дирихле (1.5) и (1.9) в произвольной ограниченной области $D$ хорошо известна (см., например, [12]).

Условие для непрерывного в области $D$ показателя $p(x)$, при выполнении которого множество гладких функций плотно в классах $W(D)$ и $W_{0}(D)$, не удавалось найти в течение длительного времени. Исследования В. В. Жикова [10] в этом направлении привели к ограничению

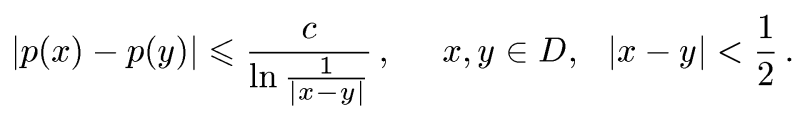


Им показано, что при условиях (1.2) и (1.12), выполненных в липшицевой области $D$, множество $C_{0}^{\infty}(D)$ плотно в классе $W(D) \cap \stackrel{\circ}{W} p_{p_{1}}(D)$ : для любой функции $u \in W(D) \cap \stackrel{\circ}{W} p_{p_{1}}(D)$ существует последовательность $\left\{u_{j}\right\} \in C_{0}^{\infty}(D)$, сходящаяся к $u(x)$ в $W_{0}(D)$, и условие (1.12) является точным для справедливости этого свойства. Из этого результата вытекает плотность множества $C^{\infty}(D) \cap W(D)$ в классе $W(D)$ для произвольной ограниченной области $D$ (см. лемму 2.2). При этом для функций класса $W_{0}(D)$ аппроксимирующая последовательность состоит из финитных бесконечно дифференцируемых в $D$ функций и в интегральном тождестве (1.4) можно ограничиться пробными функциями $\psi \in C_{0}^{\infty}(D)$.

Гёльдеровость решений уравнения (1.1) с постоянньм показателем $p(x)$ и более общих уравнений такого типа доказана О. А. Ладыженской и Н. Н. Уральцевой в работе [13]. Для уравнений вида (1.1) с гладким показателем $p(x)$ один из первых результатов о гёльдеровости решений получен П. Марчеллини в [14]. Работа [10], в которой было введено условие (1.12), стимулировала изучение качественных свойств решений уравнения (1.1) при меньших требованиях гладкости на $p(x)$. В [15] и [16] установлено, что при условии (1.12) решения уравнения (1.1) из класса

$$
W_{\text {loc }}(D)=\left\{u: u \in W_{1, \mathrm{loc}}^{1}(D),|\nabla u|^{p(x)} \in L_{1, \mathrm{loc}}(D)\right\},
$$

понимаемые в смысле интегрального тождества (1.4) на пробных функциях $\psi \in$ $W_{\text {loc }}(D)$ с компактным носителем в $D$, принадлежат пространству гёльдеровых в $D$ функций. Для гёльдерова показателя $p(x)$ этот результат ранее был получен в [17]. Точность условия (1.12) для справедливости приведенного выше утверждения вытекает из контрпримера работы [10].

Гёльдеровость решений изучена в [16] и для кусочно непрерывного показателя $p(x)$ в предположении, что область $D$ разделена гиперплоскостью $\Sigma$ на две части, в каждой из которых $p(x)$ удовлетворяет условию $(1.12)$ и скачок $p(x)$ при переходе через $\Sigma$ не обращается в нуль. Отметим, что в этом случае гладкие функции также плотны в $W(D)$ и $W_{0}(D)$. Полученные в [16] результаты обобщают работу [18], где рассмотрено уравнение с кусочно постоянным показателем $p(x)$.

Второй автор настоящей статьи изучала гёльдеровость решений уравнения (1.1) в фиксированной точке $x_{0} \in D$ в предположении, что в окрестности этой точки показатель $p(x)$ удовлетворяет условию

$$
\left|p(x)-p\left(x_{0}\right)\right| \leqslant \frac{c}{\ln \frac{1}{\left|x-x_{0}\right|}}, \quad x \in D, \quad\left|x-x_{0}\right|<\frac{1}{2} .
$$

Поскольку на характер разрыва функции $p(x)$ при $x \neq x_{0}$ не налагается никаких ограничений, то, вообще говоря, $H\left(D^{\prime}\right) \neq W\left(D^{\prime}\right)$ для любой подобласти $D^{\prime} \Subset D$. Поэтому имеет смысл рассматривать решения из классов $W_{\text {loc }}(D)$ (см. (1.13)) и $H_{\text {loc }}(D)$ : функция $u(x)$ принадлежит классу $H_{\text {loc }}(D)$, если существует последовательность $\left\{u_{j}(x)\right\} \in C^{\infty}(D)$, сходяшаяся к $u(x)$ в $W\left(D^{\prime}\right)$ в любой подобласти $D^{\prime} \Subset D$. Такие решения, которые также будем называть $W$-решениями и $H$-решениями, понимаются в смысле интегрального тождества (1.4) на соответствуюших пробных функциях $\psi \in W_{0}(D)$ и $\psi \in H_{0}(D)$ с компактным носителем в $D$. В работе [19] показано, что если $p(x)$ удовлетворяет в фиксированной точке $x_{0} \in D$ условию (1.14), то все $H$-решения и все $W$-решения уравнения (1.1) непрерывны по Гёльдеру в $x_{0}$. 
Настояшая работа посвящена исследованию граничных свойств решения обобщенной задачи Дирихле

$$
L u_{f}=0 \quad \text { в } D,\left.\quad u_{f}\right|_{\partial D}=f
$$

с непрерывной на $\partial D$ функцией $f(x)$ в предположении, что показатель суммируемости $p(x)$ удовлетворяет условиям (1.2) и (1.12). Решение данной задачи определим следуюшим образом. Продолжим граничную функцию $f \in C(\partial D)$ по непрерывности на $\bar{D}$, сохранив то же обозначение, и возьмем последовательность бесконечно дифференцируемых в $\mathbb{R}^{n}$ функций $f_{k}(x)$, которые равномерно на $\bar{D}$ сходятся к $f(x)$. Решим задачи Дирихле

$$
L u_{k}=0 \quad \text { в } D, \quad u_{k} \in W(D), \quad\left(u_{k}-f_{k}\right) \in W_{0}(D) .
$$

В теореме 4.1 доказано, что последовательность $\left\{u_{k}\right\}$ сходится равномерно на компактных подмножествах $D$ к гёльдеровой в $D$ функции $u_{f}(x)$, которая принадлежит классу $W_{\text {loc }}(D)$ и удовлетворяет уравнению (1.1). Предельная функция $u_{f}(x)$ не зависит от способов продолжения и аппроксимации граничной функции $f(x)$ и называется решением задачи Дирихле (1.15). При выводе сушествования и единственности такого решения используется принцип максимума (см. §3). В его формулировке важную роль играет понятие неравенства на гранище для функций из классов $W(D)$ и $H(D)$ (см. определение 2.1), обобщающее хорошо известное аналогичное понятие для функций из соболевского пространства. Доказательство того, что функция $u_{f}(x)$ принадлежит классу $W_{\text {loc }}(D)$ и удовлетворяет уравнению (1.1), основано на свойстве повьшенной суммируемости градиента решения (см. [8], [11]).

Для линейных равномерно эллиптических уравнений второго порядка дивергентного вида введенное вьше определение решения задачи Дирихле (1.15) содержится в обзорной статье [20]. В рассмотренном в [20] случае такое решение совпадает и с обобшенным по Винеру решением задачи Дирихле, и с обобщенным решением задачи Дирихле в смысле Пуанкаре-Перрона. Для уравнения (1.1) с постоянным показателем $p(x)$ такое определение решения задачи (1.15) ранее нам не встречалось. В монографии [21] описан другой подход к определению решения этой задачи, основанный на методе Пуанкаре-Перрона. Применение этого способа технически сложно и основано на методах теории нелинейного потенциала. Предложенная нами конструкция решения задачи (1.15) требует меньших технических усилий при доказательстве существования и единственности решения из класса $W_{\text {loc }}(D)$.

ОПРЕДЕЛЕНИЕ 1.4. Граничная точка $x_{0} \in \partial D$ называется регулярной, если

$$
\lim _{x \rightarrow x_{0}, x \in D} u_{f}(x)=f\left(x_{0}\right)
$$

для любой непрерьвной на $\partial D$ функции $f$.

В известных нам работах по исследованию поведения вблизи границы решения задачи Дирихле для уравнения (1.1) с постоянньм показателем $p(x)$ и естественных обобщений такого уравнения содержится эквивалентное данному выше понятие регулярной граничной точки: граничная точка регулярна, если решение задачи Дирихле (1.5) с любой граничной функцией $h \in C(\bar{D}) \cap W(D)$ непрерывно в этой точке. 
Критерий регулярности граничной точки для уравнения Лапласа доказан Н. Винером [22]. В работе [23] установлено, что этот критерий справедлив и для линейных равномерно эллиптических уравнений второго порядка дивергентного вида с измеримыми коэффициентами. Достаточное условие регулярности граничной точки и оценка модуля непрерывности решения вблизи границы для уравнения (1.1) с $p(x)=$ const получены В. Г. Мазьей в [24]. В работе [25] эти результаты распространены на более общие уравнения такого же типа. Необходимое условие регулярности граничной точки, совпадающее с достаточным условием при $p=2$, установлено в [26]. Критерий регулярности граничной точки в случае постоянного показателя $p(x)$ доказан в работе [27] (частный случай см. в более ранней работе [28]), где показано, что необходимое условие совпадает с уже известным достаточным.

Важную роль в граничных свойствах решений играет понятие емкости компакта. Прежде чем его ввести, обозначим через $B_{R}$ открытый шар радиуса $R$ и будем считать, что $p(x)$ удовлетворяет в $B_{R}$ условию (1.2).

ОПРЕДЕЛЕНИЕ 1.5. Пусть $K \subset B_{R}$-компакт. Число

$$
C_{p}\left(K, B_{R}\right)=\inf \int_{B_{R}} \frac{|\nabla \varphi|^{p(x)}}{p(x)} d x,
$$

где точная нижняя грань берется по множеству функций $\varphi \in C_{0}^{\infty}\left(B_{R}\right)$ таких, что $\varphi \geqslant 1$ на $K$, называется $p$-емкостью $K$ относительно шара $B_{R}$.

В теореме 5.1 показано, что точная нижняя грань в (1.17) достигается на единственной функции $u \in H_{0}\left(B_{R}\right)$, которая называется емкостным потенциалом компакта $K$. Емкостный потенциал удовлетворяет уравнению (1.9) с мерой $\mu$, сосредоточенной на $\partial K$, такой, что $\mu(K)=C_{p}\left(K, B_{R}\right)$. Данные утверждения имеют место при одном только условии (1.2) на показатель суммируемости $p(x)$.

Приведенное вьше определение емкости для $p(x)=$ const восходит к работе [29]. В. А. Кондратьевым в случае $p=2[30]$ предложено другое эквивалентное определение емкости, в том числе и применительно к уравнениям высокого порядка.

Сушествование и единственность емкостного потенциала для $p(x)=$ const следует из обших соображений выпуклого анализа (см., например, [31]).

Сформулируем основные результаты о регулярности граничной точки. Продолжим $p(x)$ на все $\mathbb{R}^{n}$ с сохранением свойств (1.2), (1.12) и положим для $x_{0} \in \partial D$

$$
p_{0}=p\left(x_{0}\right), \quad \gamma(t)=\left(C_{p}\left(\bar{B}_{t}^{x_{0}} \backslash D, B_{2 t}^{x_{0}}\right) t^{p_{0}-n}\right)^{1 /\left(p_{0}-1\right)},
$$

где $B_{t}^{x_{0}}$ - открытый шар радиуса $t$ с центром в $x_{0}$.

Теорема 1.1. Для регулярности граничной точки $x_{0} \in \partial D$ необходимо и достаточно расходимости в нуле интеграла

$$
\int_{0} \gamma(t) t^{-1} d t=\infty
$$

Если $p_{0}>n$, то емкость точки $x_{0}$ относительно шара $B_{t}^{x_{0}}$ удовлетворяет неравенству $C_{p}\left(\left\{x_{0}\right\}, B_{t}^{x_{0}}\right) \geqslant C(n, p) t^{n-p_{0}}>0($ см. $\S 5)$ и граничная точка $x_{0}$ всегда регулярна.

В следуюшей теореме дается оценка модуля непрерывности решения в регулярной граничной точке. Ниже $M=\max _{x \in \partial D}|f(x)|$. 
Теорема 1.2. Существуют положительные постоянные $\theta$ и $С$, зависящие только от $n, p$ и $M$, такие, что при $r \leqslant \rho / 4$ справедливо неравенство

$$
\sup _{D \cap B_{r}^{x_{0}}}\left|u_{f}(x)-f\left(x_{0}\right)\right| \leqslant C\left(\underset{\partial D \cap B_{\rho}^{x_{0}}}{\operatorname{osc}} f+\rho+\underset{\partial D}{\operatorname{osc}} f \exp \left(-\theta \int_{r}^{\rho} \gamma(t) t^{-1} d t\right)\right),
$$

если $p_{0} \leqslant n$, и неравенство

$$
\sup _{D \cap B_{r}^{x_{0}}}\left|u_{f}(x)-f\left(x_{0}\right)\right| \leqslant \underset{\partial D \cap B_{\rho}^{x_{0}}}{\operatorname{osc}} f+C\left(\frac{r}{\rho}\right)^{1-n / p_{0}} \underset{\partial D}{\operatorname{Osc} f},
$$

если $p_{0}>n$.

Если $p(x)=$ const, то критерий регулярности граничной точки (1.19), как уже отмечалось вьше, получен в работе [27], а оценки модуля непрерывности решения (1.20) и (1.21) в регулярной граничной точке - в работе [24]. Важную роль в доказательстве теорем 1.1 и 1.2 играет неравенство Харнака для неотрицательных суперрешений уравнения (1.1) (см. лемму 6.4), которое в случае $p(x)=$ const установлено в [32].

Следующее достаточное условие на гранищу области $D$ в окрестности точки $x_{0} \in \partial D$, обеспечивающее гёльдеровость решения в $x_{0}$ и имеющее смысл при $p_{0} \leqslant n$, является следствием (1.20) и оценок емкости замкнутого шара, полученных в $§ 5$.

ТЕОрема 1.3. Если граничная функиия $f(x)$ удовлетворяет условию Гёльдера в точке $x_{0} \in \partial D$ и внешность области $D$ в окрестности $x_{0}$ содержит конус с вершиной в $x_{0}$, то решение $u_{f}(x)$ непрерывно по Гёльдеру в $x_{0}$.

Приведем еше геометрические условия регулярности граничной точки, которую будем считать совпадающей с началом координат $O$. Предположим, что внутренность дополнения области $D$ в окрестности точки $O$ совпадает с областью вида

$$
\left\{x: 0<x_{n}<a, \sum_{i=1}^{n-1} x_{i}^{2}<g^{2}\left(x_{n}\right)\right\}
$$

где $g(t)$ - непрерывная, неубывающая функция, удовлетворяющая для $\alpha>1$ и $t \in$ $[0, a]$ неравенствам $t^{\alpha} \leqslant g(t) \leqslant t$.

ТеОРема 1.4. Если $n-1 \leqslant p_{0} \leqslant n$, то граничная точка О регулярна, а если $p_{0}<n-1$, то для ее регулярности необходимо и достаточно расходимости в нуле интеграла

$$
\int_{0}\left(\frac{g(t)}{t}\right)^{\frac{n-1-p_{0}}{p_{0}-1}} t^{-1} d t=\infty
$$

Отметим, что если $g(t) \geqslant C t$, то область $D$ удовлетворяет в точке $O \in \partial D$ условию внешнего конуса Пуанкаре и условие (1.19) выполнено при любом значении $p_{0}$. В случае $p(x)=$ const утверждение теоремы 1.4 содержится в работе [24].

Полученные результаты остаются в силе и для решений уравнения

$$
\sum_{i, j=1}^{n} \frac{\partial}{\partial x_{i}}\left(a_{i j}(x)|\nabla u|^{p(x)-2} \frac{\partial u}{\partial x_{j}}\right)=0
$$

с измеримой, симметрической и равномерно положительно определенной в $D$ матрицей $\left\|a_{i j}(x)\right\|$. 


\section{§2. Сведения о классах $W(D)$ и $W_{0}(D)$}

1. Плотность гладких функций в классах $W(D)$ и $W_{0}(D)$. Ниже, не оговаривая этого особо, будем считать, что измеримьй показатель суммируемости $p(x)$, помимо прочих ограничений, удовлетворяет условию (1.2).

Сначала рассмотрим случай, когда функция $u \in W_{0}(D)$ имеет компактный носитель в $D$. Продолжим $u(x)$ нулем вне $D$ и с помощью ядра

$$
\omega \geqslant 0, \quad \omega \in C_{0}^{\infty}\left(\mathbb{R}^{n}\right), \quad \omega(x) \equiv 0 \text { при }|x| \geqslant 1, \quad \int_{\mathbb{R}^{n}} \omega(x) d x=1,
$$

определим усредненные функции

$$
u_{\varepsilon}(x)=\int_{D} u(y) \omega_{\varepsilon}(x-y) d y, \quad \omega_{\varepsilon}(x)=\varepsilon^{-n} \omega\left(\varepsilon^{-1} x\right) .
$$

ЛЕмма 2.1 [10]. Если выполнено условие (1.12), то для любой функиии $u \in W_{0}(D)$ с компактным носителем в $D$ существует последовательность ее усреднений $\left\{u_{\varepsilon_{j}}\right\} \in C_{0}^{\infty}(D)$, сходящаяся в $W_{0}(D) \kappa u(x)$ при $\varepsilon_{j} \rightarrow 0$.

Приведем еще одно вспомогательное утверждение, в котором характеризуется показатель степени суммируемости функций из классов $W_{0}(D)$ и $W_{\mathrm{loc}}(D)$.

ПРЕДЛОЖЕНИЕ 2.1. Если показатель суммируемости $p(x)$ равномерно непрерывен в $D$, то существует постоянная $\gamma=\gamma(n)>1$ такая, что:

a) ecлu $u \in W_{0}(D)$, mo $|u|^{\gamma p(x)} \in L_{1}(D)$;

б) $е с л u \quad u \in W_{\mathrm{loc}}(D)$, mo $|u|^{\gamma p(x)} \in L_{1, \mathrm{loc}}(D)$.

ДокАЗАТЕЛьСтво. Докажем свойство а). Продолжим функцию $u \in W_{0}(D)$ нулем в $\mathbb{R}^{n} \backslash D$ и рассмотрим $u(x)$ в произвольном шаре $B_{r}$ с центром на $\bar{D}$, радиус $r=r(n, p)$ которого столь мал, что

$$
\begin{gathered}
\gamma p(x) \leqslant s k, \quad x \in B_{r} \cap D, \quad k=\frac{n}{n-1}, \\
\gamma=\frac{n^{2}}{n^{2}-1}, \quad s=\inf _{B_{r} \cap D} p(x) .
\end{gathered}
$$

По теореме вложения Соболева имеем

$$
\left(\int_{B_{r}}|u|^{k s} d x\right)^{1 / k} \leqslant C\left(\int_{B_{r}}|\nabla u|^{s} d x+\int_{B_{r}}|u| d x\right) .
$$

Поэтому $|u|^{\gamma p(x)} \in L_{1}\left(B_{r}\right)$ в силу неравенства из (2.1) и свойство а) вытекает из произвольности шара $B_{r}$.

Для доказательства свойства б) достаточно заметить, что если $u \in W_{\text {loc }}(D)$, то функция $|u|^{\gamma p(x)}$ суммируема в шарах $B_{r} \Subset D$, радиус которых удовлетворяет условию (2.1). Предложение доказано.

Таким образом, для равномерно непрерывного в $D$ показателя суммируемости $p(x)$ в интегральном тождестве (1.4) для $W$-решений и $H$-решений можно использовать пробные функции вида $\psi(x)=u(x) \eta(x)$, где $u(x)$ - соответствуюшее решение уравнения (1.1), а $\eta \in C_{0}^{\infty}(D)$. 
ЛЕмма 2.2. Если выполнено условие (1.12), то справедливы следующие утверждения:

а) мнохество $C^{\infty}(D) \cap W(D)$ плотно в $W(D)$ относительно сходимости в $W(D)$;

б) мнохество $C_{0}^{\infty}(D)$ плотно в $W_{0}(D)$ относительно сходимости в $W_{0}(D)$.

ДокаЗАТЕльство. Пусть $u \in W(D),\left\{\mathcal{B}_{r_{j}}\right\}$ - локально конечное покрытие области $D$ шарами $\overline{\mathcal{B}}_{r_{j}} \subset D, j=1,2, \ldots$, и $\left\{\eta_{j}\right\}$ - подчиненное этому покрытию разбиение единищы. Кратность покрытия будем считать конечной, зависяшей только от размерности пространства $n$. Обозначим $u_{j}=\eta_{j} u$ и рассмотрим усреднение $u_{j}$, положив

$$
w_{j}(x)=\int_{D} \omega_{\varepsilon_{j}}(x-y) u_{j}(y) d y .
$$

Радиус усреднения $\varepsilon_{j}$ выберем столь малым, что $w_{j} \in C_{0}^{\infty}\left(\mathcal{B}_{r_{j}}\right)$. Согласно утверждению предложения $2.1 u_{j} \in W_{0}(D)$. Поэтому в силу леммы 2.1 и свойства усреднения по заданному $\varepsilon \in(0,1 / 2)$ найдется $\varepsilon_{j}$, для которого

$$
\int_{D}\left(\left|u_{j}-w_{j}\right|+\left|\nabla u_{j}-\nabla w_{j}\right|^{p(x)}\right) d x \leqslant \varepsilon^{j} .
$$

Положим $w=\sum_{j} w_{j}$. Ясно, что $w \in C^{\infty}(D)$. На любом открытом множестве $G, \bar{G} \subset D$, имеет место равенство $u=\sum_{j} u_{j}$, в котором сумма содержит конечное число слагаемых. Пусть $\widetilde{G}$ - объединение шаров $\mathcal{B}_{r_{j}}$, имеющих непустое пересечение с $G$. Из неравенства $(2.2)$ и конечной кратности покрытия имеем

$\int_{G}\left(|u-w|+|\nabla u-\nabla w|^{p(x)}\right) d x \leqslant C \sum_{j} \int_{\widetilde{G}}\left(\left|u_{j}-w_{j}\right|+\left|\nabla u_{j}-\nabla w_{j}\right|^{p(x)}\right) d x \leqslant C \varepsilon$

с постоянной $C$, зависяшей только от $n$ и $p$. Отсюда свойство а) следует из произвольности $G$ и $\varepsilon$.

Свойство б) вытекает из утверждения леммы 2.1 и определения класса $W_{0}(D)$. Лемма доказана.

Пользуясь приведенными выше рассуждениями, нетрудно показать, что при условии (1.12) множество $C^{\infty}(D)$ плотно и в классе $W_{\text {loc }}(D)$ относительно сходимости на открытых строго внутренних подмножествах $D^{\prime}$ области $D$ :

$$
\forall u \in W_{\mathrm{loc}}(D) \quad \exists u_{j} \in C^{\infty}(D): u_{j} \rightarrow u \text { в } W\left(D^{\prime}\right) \quad \forall D^{\prime} \Subset D .
$$

2. Свойства классов $W(D)$ и $H(D)$. Класс $W_{0}(D)$ можно определить и другим способом. При доказательстве эквивалентности обоих определений нам потребуется теорема Шеффе.

ТЕОРема 2.1. Если последовательность $\left\{w_{j}\right\}$, состоящая из неотрицательных, суммируемых в $D$ функиий, сходится $\kappa$ функиии $w \in L_{1}(D)$ почти всюду в $D u$

$$
\lim _{j \rightarrow \infty} \int_{D} w_{j} d x=\int_{D} w d x
$$

mo $w_{j} \rightarrow w$ в $L_{1}(D)$. 
ЛЕмма 2.3. Для принадлежности функиии и $(x)$ классу $W_{0}(D)$ необходимо и достаточно существования последовательности $\left\{u_{j}\right\}$, состоящей из функций $u_{j} \in \stackrel{\circ}{W} \underset{1}{1}(D)$ с компактным носителем в $D$, такой, что

$$
u_{j} \rightarrow u \quad \text { в } \quad \stackrel{\circ}{W_{1}^{1}}(D), \quad\left|\nabla u_{j}\right|^{p(x)} \rightarrow|\nabla u|^{p(x)} \quad \text { в } \quad L_{1}(D) .
$$

ДокАЗАТЕЛЬСТво. Достаточность. Если вьполнены свойства (2.3), то, переходя к подпоследовательности, можно считать, что $\left|\nabla u_{j}\right|^{p} \rightarrow|\nabla u|^{p}$ почти всюду в $D$, и из теоремы Шеффе вытекает равностепенная интегрируемость последовательности $\left\{\left|\nabla u_{j}\right|^{p}\right\}$. Но тогда равностепенно интегрируема последовательность $\left\{\left|\nabla u-\nabla u_{j}\right|^{p}\right\}$, сходящаяся к нулю почти всюду в $D$, и нужное утверждение вытекает из теоремы Лебега. Достаточность доказана.

Необходимость. Пусть $u \in W_{0}(D)$. Тогда существует последовательность функций $u_{j} \in W(D)$ с компактным носителем в $D$, которая сходится к $u(x)$ в $W(D)$. Поскольку сходимость в $W(D)$ влечет сходимость в $\stackrel{\circ}{W}=1(D)$, то достаточно установить, что $\left|\nabla u_{j}\right|^{p(x)} \rightarrow|\nabla u|^{p(x)}$ в $L_{1}(D)$. Пользуясь неравенством

$$
\left.|| \xi\right|^{p}-|\zeta|^{p}|\leqslant p| \xi-\zeta \mid\left(|\xi|^{p-1}+|\zeta|^{p-1}\right)
$$

и неравенством Юнга, будем иметь

$$
\begin{aligned}
\left.\int_{D}|| \nabla u\right|^{p(x)}-\left|\nabla u_{j}\right|^{p(x)} \mid d x \leqslant & C(\varepsilon, p) \int_{D}\left|\nabla u-\nabla u_{j}\right|^{p(x)} d x \\
& +\varepsilon \int_{D}\left(|\nabla u|^{p(x)}+\left|\nabla u_{j}\right|^{p(x)}\right) d x .
\end{aligned}
$$

Поскольку последовательность $\left\{\left|\nabla u_{j}\right|^{p}\right\}$ равномерно ограничена в $L_{1}(D)$, то ввиду произвольности $\varepsilon$ приходим к требуемому свойству из (2.3). Необходимость доказана. Лемма доказана.

Аналогичньй факт имеет место и для класса $W(D)$ : для принадлежности функции $u(x)$ классу $W(D)$ необходимо и достаточно существования последовательности $\left\{u_{j}\right\}$, удовлетворяющей свойствам

$$
u_{j} \in W_{1}^{1}(D), \quad u_{j} \rightarrow u \text { в } W_{1}^{1}(D), \quad\left|\nabla u_{j}\right|^{p(x)} \rightarrow|\nabla u|^{p(x)} \text { в } L_{1}(D) .
$$

Полученные результаты нетрудно перенести на классы $H_{0}(D)$ и $H(D)$. В этом случае последовательность $\left\{u_{j}\right\}$ из $(2.3)$ принадлежит множеству $C_{0}^{\infty}(D)$, а последовательность из $(2.6)$ - множеству $C^{\infty}(D) \cap W_{1}^{1}(D)$.

Из оценки (2.5) вытекает одно свойство сходящейся в $W_{0}(D)$ последовательности, которое нам потребуется в дальнейшем.

ПРЕДЛОЖЕНИЕ 2.2. Если последовательность функций $u_{j} \in W_{0}(D)$ сходится в $W_{0}(D) \kappa$ функции $u \in W_{0}(D)$, mо $\left|\nabla u_{j}\right|^{p} \rightarrow|\nabla u|^{p}$ в $L_{1}(D)$.

ЛЕмма 2.4. Классы $W(D)$ и $H(D)$ полны относительно сходимости в $W(D)$, а классы $W_{0}(D)$ и $H_{0}(D)$ - относительно сходимости в $W_{0}(D)$. 
ДокАЗАТЕЛЬСТво. Покажем полноту класса $W(D)$. Пусть $\left\{u_{j}\right\}$ - фундаментальная последовательность. Тогда по заданному $\varepsilon>0$ найдется номер $N$ такой, что

$$
\int_{D}\left(\left|u_{i}-u_{j}\right|+\left|\nabla u_{i}-\nabla u_{j}\right|^{p(x)}\right) d x \leqslant \varepsilon, \quad i, j>N .
$$

В частности, последовательность $\left\{u_{i}\right\}$ фундаментальна в $W_{1}^{1}(D)$, и существует функция $u \in W_{1}^{1}(D)$, к которой $\left\{u_{i}\right\}$ сходится в $W_{1}^{1}(D)$. Переходя к подпоследовательности, можно считать, что $\left|u-u_{i}\right|+\left|\nabla\left(u-u_{i}\right)\right| \rightarrow 0$ почти всюду в $D$ и по лемме Фату из (2.7) придем к неравенству

$$
\int_{D}\left(\left|u-u_{j}\right|+\left|\nabla u-\nabla u_{j}\right|^{p(x)}\right) d x \leqslant \varepsilon, \quad i>N,
$$

из которого следует, что $u_{j} \rightarrow u$ в $W(D)$ и $u \in W(D)$. Точно так же устанавливается полнота других классов. Лемма доказана.

Если $u \in W(D)$, то отрицательная и положительная части функции $u(x)$

$$
u^{-}(x)=\min (u(x), 0), \quad u^{+}(x)=\max (u(x), 0)
$$

также принадлежат классу $W(D)$. Аналогичный факт справедлив и для усеченных функций вида

$$
u_{m}^{-}(x)=\min (u(x), m), \quad u_{m}^{+}(x)=\max (u(x), m) .
$$

Проверим выполнение этих свойств в классах $H(D), W_{0}(D)$ и $H_{0}(D)$. Предварительно докажем следующее вспомогательное утверждение.

ЛЕмма 2.5. Предположим, ито функиия $u \in W(D)$ является пределом последовательности $\left\{u_{j}\right\} \in W(D)$, сходящейся $к u(x)$ в $W(D)$. Тогда существует последовательность $\left\{v_{j}\right\} \in W(D)$, которая сходится $\kappa u_{m}^{-}(x)$ в $W(D)$ и удовлетворяет следующим свойствам:

а) $v_{j}(x) \leqslant m$ почти всюду в $D$;

б) если $u_{j} \in C^{\infty}(D)$, mo $v_{j} \in C^{\infty}(D)$;

в) если $m \geqslant 0$ и функиии $u_{j}(x)$ имеют компактный носитель в $D$, то функиии $v_{j}(x)$ также обладают этим свойством;

г) если $u_{j}(x) \geqslant m \geqslant 0$ почти всюду на множсестве $E \subset D$, mо $v_{j}(x)=m$ почти всюду на $E$.

ДОКАЗАТЕЛЬСТвО. Пусть $\eta_{k}(t) \in C^{\infty}\left(\mathbb{R}^{1}\right), k=1,2, \ldots, \eta(t)=1$ при $t<$ $-2^{-k}+m, \eta(t)=0$ при $t \geqslant m, 0 \leqslant \eta(t) \leqslant 1$ и $\left|\eta^{\prime}(t)\right| \leqslant$ const $\cdot 2^{k}$. Рассмотрим еше последовательность $\left\{g_{k}(t)\right\}$ непрерьвно дифференцируемых на числовой прямой функций, задаваемых равенствами

$$
g_{k}(t)=-\left((t-m)^{2}+2^{-2 k}\right)^{1 / 2}+2^{-k} \text { при } t<m, \quad g_{k}(t)=0 \text { при } t \geqslant m,
$$

и положим $f_{k}(t)=g_{k}(t) \eta_{k}(t)+m$. Как нетрудно видеть, $f_{k}(t) \in C^{\infty}\left(\mathbb{R}^{1}\right)$. Простой проверкой получим, что последовательность $\left\{f_{k}\left(u_{j}\right)\right\}$ состоит из функций класса 
$W(D)$, обладающих свойствами а) - г) утверждения леммы. Осталось установить, что при соответствующем выборе $k$ и $j$ выражение

$$
I_{k, j}=\int_{D}\left(\left|f_{k}\left(u_{j}\right)-u_{m}^{-}\right|+\left|\nabla f_{k}\left(u_{j}\right)-\nabla u_{m}^{-}\right|^{p(x)}\right) d x
$$

можно сделать сколь угодно малым. Поскольку $\left|f_{k}^{\prime}(t)\right| \leqslant$ const, то, как нетрудно видеть,

$$
\begin{aligned}
I_{k, j} \leqslant & C(p)\left(\int_{D}\left(\left|u_{j}-u\right|+\left|\nabla u_{j}-\nabla u\right|^{p(x)}\right) d x\right. \\
& +\int_{D}\left(\left|f_{k}(u)-u_{m}^{-}\right|+\left|f_{k}^{\prime}(u) \nabla u-\nabla u_{m}^{-}\right|^{p(x)}\right) d x \\
& \left.+\int_{D}\left|f_{k}^{\prime}\left(u_{j}\right)-f_{k}^{\prime}(u)\right|^{p(x)}|\nabla u|^{p(x)} d x\right) \\
= & C(p)\left(I_{j}^{(1)}+I_{k}^{(2)}+I_{k, j}^{(3)}\right) .
\end{aligned}
$$

Оценим каждое слагаемое в правой части (2.8). Ясно, что $I_{j}^{(1)} \rightarrow 0$ при $j \rightarrow \infty$. Для почти всех точек множества $\{x: u(x) \geqslant m\}$ выполнены равенства $u_{m}^{-}(x)=m$, $\nabla u_{m}^{-}(x)=0, f_{k}(u(x))=m$ и $f_{k}^{\prime}(u(x))=0$. Поэтому, полагая $G_{\delta}=\{x: m-\delta<$ $u<m\}, F_{\delta}=\{x: u \leqslant m-\delta\}$, где $\delta>0$, и учитывая, что $\left|f_{k}^{\prime}(t)\right| \leqslant$ const, будем иметь

$$
\begin{aligned}
I_{k}^{(2)} \leqslant & \int_{G_{\delta}}\left(\left|f_{k}(u)-u\right|\right) d x+C(p) \int_{G_{\delta}}|\nabla u|^{p(x)} d x \\
& +\int_{F_{\delta}}\left(\left|f_{k}(u)-u\right|+\left|f_{k}^{\prime}(u)-1\right|^{p(x)}|\nabla u|^{p(x)}\right) d x .
\end{aligned}
$$

Первые два интеграла в правой части (2.9) стремятся к нулю при $\delta \rightarrow 0$, а так как $f_{k}^{\prime}(t) \rightarrow 1$ при $k \rightarrow \infty$ для всех $t \leqslant m-\delta$, то $I_{k}^{(2)} \rightarrow 0$ при $k \rightarrow \infty$ и по заданному $\varepsilon$ найдется номер $k_{0}(\varepsilon)$ такой, что $I_{k_{0}}^{(2)} \leqslant \varepsilon$.

Зафиксируем номер $k_{0}(\varepsilon)$ и рассмотрим оставшийся интеграл $I_{k_{0}, j}^{(3)}$. Переходя к подпоследовательности, можно считать, что $u_{j} \rightarrow u$ почти всюду в $D$. Функции $f_{k}^{\prime}(t)$ непрерьвны и последовательность $\left\{f_{k_{0}}^{\prime}\left(u_{j}\right)\right\}, j=1,2, \ldots$, тоже сходится почти всюду в $D$ к функции $f_{k_{0}}^{\prime}(u(x))$. Таким образом, $I_{k_{0}, j}^{(3)} \rightarrow 0$ при $j \rightarrow \infty$, и существует номер $j_{0}=j_{0}\left(k_{0}, \varepsilon\right)$, для которого $I_{j}^{(1)}+I_{k_{0}}^{(2)}+I_{k_{0}, j_{0}}^{(3)} \leqslant \varepsilon$. В результате, с учетом (2.8), придем к требуемому неравенству $I_{k_{0}, j_{0}} \leqslant C \varepsilon$. Лемма доказана.

Замкнутость класса $H(D)$ относительно операции срезки вытекает из определения $H(D)$ и свойства б) леммы 2.5. Если же $u \in W_{0}(D) \quad\left(u \in H_{0}(D)\right)$, то $u_{m}^{-} \in$ $W_{0}(D)$ (соответственно, $u_{m}^{-} \in H_{0}(D)$ ) при $m \geqslant 0$ и $u_{m}^{+} \in W_{0}(D)$ (соответственно, $\left.u_{m}^{+} \in H_{0}(D)\right)$ при $m \leqslant 0$. Действительно, если $u \in W_{0}(D)$, то по определению класса $W_{0}(D)$ найдется последовательность $u_{j} \in W(D)$, состояшая из функций с компактным носителем, которая сходится к $u(x)$ в $W_{0}(D)$. Отсюда в силу свойства в) последовательности $\left\{v_{j}\right\}$ вытекает, что $u_{m}^{-} \in W_{0}(D)$ при $m \geqslant 0$. Соответствуюшее свойство для $u_{m}^{+}(x)$ следует из равенства $u_{m}^{+}(x)=-\min (-u(x),-m)$. Аналогичными рассуждениями разбирается и случай, когда $u \in H_{0}(D)$. 
3. Неравенства для функций из классов $W(D)$ и $H(D)$. При изучении граничных свойств решения задачи Дирихле важную роль играет понятие неравенства на границе для функций из классов $W(D)$ и $H(D)$. Следуя работе [33], определим это понятие.

ОПРЕДЕЛЕНИЕ 2.1. Функция $u \in W(D)(u \in H(D))$ удовлетворяет неравенству $u \geqslant 0$ на границе $\partial D$ в смисле $W(D)$ (соответственно, в смисле $H(D)$ ), если ее отрицательная часть $u^{-}(x)$ принадлежит классу $W_{0}(D)$ (соответственно, классу $H(D))$.

Если функция $u(x)$, принадлежашая одному из классов $W(D)$ или $H(D)$, непрерывна в замкнутой области $\bar{D}$ и $u(x) \geqslant 0$ на $\partial D$ в обычном смысле, то $u \geqslant 0$ на $\partial D$ и в соответствуюшем смысле данного определения.

$\mathrm{K}$ введенному понятию неравенства на гранище $\partial D$ (в смысле $W(D)$ или $H(D)$ ) сводятся и другие типы неравенств. Например, $u \leqslant 0$ на $\partial D$, если $-u \geqslant 0$ на $\partial D$; функции $u(x)$ и $v(x)$ из одного и того же класса удовлетворяют неравенству $u \geqslant v$ на $\partial D$ в соответствуюшем смысле, если $u-v \geqslant 0$ на $\partial D$; эти же функции удовлетворяют равенству $u=v$ на $\partial D$, если $u-v \geqslant 0$ на $\partial D$ и $v-u \geqslant 0$ на $\partial D$;

$$
\inf _{\partial D} u=\sup \{k: u \geqslant k \text { на } \partial D\}, \quad \sup _{\partial D} u=-\inf _{\partial D}(-u) .
$$

Неравенства для функций из $W(D)$ и $H(D)$ на границе $\partial D$ могут быть обобщены. С этой целью определим классы $W_{0}(D, E)$ и $H_{0}(D, E)$, связанные с подмножествами $E$ замкнутой области $\bar{D}$. Класс $W_{0}(D, E)\left(H_{0}(D, E)\right)$ состоит из функций $u \in W(D)$ (соответственно, функций $u \in H(D)$ ), для каждой из которых существует последовательность $\left\{u_{j}\right\} \in W(D)$ (соответственно, $\left\{u_{j}\right\} \in H(D)$ ), состоящая из функций с носителем вне окрестности $E \cap \bar{D}$ и сходящаяся к $u(x)$ в $W(D)$. В частности, если $E=\partial D$, то $W_{0}(D, E)=W_{0}(D)$ и $H_{0}(D, E)=H_{0}(D)$.

Функция $u \in W(D)(u \in H(D))$ удовлетворяет неравенству $u \geqslant 0$ на $E \subset \bar{D}$ в смысле $W(D)$ (соответственно, в смысле $H(D)$ ), если ее отрицательная часть $u^{-}(x)$ принадлежит классу $W_{0}(D, E)$ (соответственно, классу $\left.H_{0}(D, E)\right)$. Для непрерывной на $\bar{E}$ функции из класса $W(D)$ или $H(D)$ соответствуюшее определение выполнено, если $u(x) \geqslant 0$ на $E$ в обычном смысле. Определения других типов неравенств на $E$ аналогичны данным вьше.

Приведем одно вспомогательное утверждение, которое нам понадобится далее.

ПРЕДЛОЖЕНИЕ 2.3. Для выполнения равенства и(x)=1 на компакте $K \subset B_{R}$ в смысле $H\left(B_{R}\right)$ достаточно существования последовательности $\left\{u_{j}\right\} \in C_{0}^{\infty}\left(B_{R}\right)$ такой, что $u_{j} \rightarrow u$ в $W_{0}\left(B_{R}\right)$ u $u_{j}(x)=1$ в окрестности $K$.

ДокАЗАТЕЛЬСТво. Если последовательность $\left\{u_{j}\right\} \in C_{0}^{\infty}\left(B_{R}\right)$ удовлетворяет условиям леммы, то последовательность $\left\{u_{j}(x)-1\right\}$ сходится в $W\left(B_{R}\right)$ к функции $u(x)-1$. Поскольку $u_{j}(x)-1=0$ в окрестности $K$, то $(u-1)^{-} \in H_{0}\left(B_{R}, K\right)$ по свойствам б) и г) леммы 2.5. Точно так же можно показать, что $(1-u)^{-} \in$ $H_{0}\left(B_{R}, K\right)$. Предложение доказано. 


\section{§3. Разрешимость задачи Дирихле в классах $W(D)$ и $H(D)$}

1. Вспомогательные результаты. Сначала рассмотрим вариационные задачи (1.7) и (1.8). В предположении (1.2) интегрант $|\xi|^{p(x)}$ является строго выпуклой функцией переменной $\xi \in \mathbb{R}^{n}$. Условие выпуклости будем использовать либо в виде

$$
|\xi|^{p}-|\zeta|^{p} \geqslant p|\zeta|^{p-2} \zeta \cdot(\xi-\zeta),
$$

где точка означает скалярное произведение, либо в виде неравенства Кларксона (см. [34]): если $p \geqslant 2$, то

$$
|\xi|^{p}-|\zeta|^{p} \geqslant p|\zeta|^{p-2} \zeta \cdot(\xi-\zeta)+\frac{|\xi-\zeta|^{p}}{2^{p-1}-1}
$$

а если $1<p<2$, то

$$
|\xi|^{p}-|\zeta|^{p} \geqslant p|\zeta|^{p-2} \zeta \cdot(\xi-\zeta)+\frac{3 p(p-1)}{16} \frac{|\xi-\zeta|^{2}}{(|\xi|+|\zeta|)^{2-p}}
$$

для $\xi$ и $\zeta$, не обращающихся в нуль одновременно.

Кроме того, нам потребуются еще два вспомогательных неравенства.

ПрЕДЛОЖЕНИЕ 3.1. Если $1<p_{1} \leqslant p \leqslant p_{2}<\infty$, то существует постоянная $\delta \in(0,1]$, зависящая только от $p$, такая, что для любых $\xi, \zeta \in \mathbb{R}^{n} u|t| \leqslant 1$ имеет место неравенство

$$
\left|\left(|\xi+t \zeta|^{p-2}(\xi+t \zeta)-|\xi|^{p-2} \xi\right) \cdot \zeta\right| \leqslant C(n, p)|t|^{\delta}\left(|\xi|^{p}+|\zeta|^{p}\right) .
$$

ДокАЗАТЕЛЬСТво. Не ограничивая обшности, можно считать $\xi \neq 0, t \in(0,1]$. Если $|\xi|<4 t|\zeta|$, то доказательство очевидно, и будем предполагать, что

$$
|\xi| \geqslant 4 t|\zeta| \text {. }
$$

В этом случае

$$
\left|\xi+\tau^{\prime} \zeta\right| \geqslant|\xi|-\tau^{\prime}|\zeta| \geqslant \frac{3|\xi|}{4} \quad \forall \tau^{\prime} \in[0, t] .
$$

Поэтому при фиксированных $\xi, \zeta \in \mathbb{R}^{n}, \xi \neq 0$, функция переменного $\tau$

$$
g(\tau)=|\xi+\tau \zeta|^{p-2}(\xi+\tau \zeta) \cdot \zeta
$$

непрерывно дифференцируема на отрезке $[0, t]$ для любого $p \in\left[p_{1}, p_{2}\right]$ и по теореме Лагранжа сушествует точка $\tau^{\prime} \in(0, t)$, для которой $g(t)-g(0)=t g^{\prime}\left(\tau^{\prime}\right)$, т. е.

$$
\left|\left(|\xi+t \zeta|^{p-2}(\xi+t \zeta)-|\xi|^{p-2} \xi\right) \cdot \zeta\right| \leqslant C(n, p)|t||\zeta|^{2}\left|\xi+\tau^{\prime} \zeta\right|^{p-2} .
$$

Если теперь $p \geqslant 2$, то согласно (3.5) и неравенству Юнга имеем

$$
\left|\left(|\xi+t \zeta|^{p-2}(\xi+t \zeta)-|\xi|^{p-2} \xi\right) \cdot \zeta\right| \leqslant C(n, p)|t|\left(|\xi|^{p}+|\zeta|^{p}\right) .
$$

Если же $1<p_{1} \leqslant p<2$, то $p>1+\delta$ для $\delta=\left(p_{1}-1\right) / 2$ и в силу $(3.5)-(3.7)$ и неравенства Юнга

$$
\begin{aligned}
\left|\left(|\xi+t \zeta|^{p-2}(\xi+t \zeta)-|\xi|^{p-2} \xi\right) \cdot \zeta\right| & \leqslant C(n, p)|t|^{\delta}|\zeta|^{\delta+1}|\xi|^{p-1-\delta} \\
& \leqslant C(n, p)|t|^{\delta}\left(|\xi|^{p}+|\zeta|^{p}\right) .
\end{aligned}
$$

Предложение доказано. 
ПРЕДЛОЖЕНИЕ 3.2. Если $1<p_{1} \leqslant p \leqslant p_{2}<\infty$, то для заданного $\varepsilon>0$ и любых $\xi, \zeta, \eta \in \mathbb{R}^{n}$ имеет место неравенство

$$
\left|\left(|\xi|^{p-2} \xi-|\zeta|^{p-2} \zeta\right) \cdot \eta\right| \leqslant C(n, p, \varepsilon)|\xi-\zeta|^{p}+\varepsilon\left(|\zeta|^{p}+|\eta|^{p}\right) .
$$

ДоКАЗАТЕЛЬСТво. Если $|\zeta|<4|\xi-\zeta|$, то, полагая, что $\xi=\zeta+(\xi-\zeta)$, легко придем к (3.8) по неравенству Юнга. Случай $\zeta=0$ также тривиален. Поэтому будем предполагать

$$
|\zeta| \geqslant 4|\xi-\zeta|, \quad \zeta \neq 0,
$$

что влечет неравенство

$$
|\zeta+t(\xi-\zeta)| \geqslant|\zeta|-t|\xi-\zeta| \geqslant \frac{3}{4}|\zeta| \quad \forall t \in[0,1] .
$$

В силу $(3.2)$ и предположения $\zeta \neq 0$ функция

$$
g(t)=|\zeta+t(\xi-\zeta)|^{p-2}(\zeta+t(\xi-\zeta)) \cdot \eta
$$

непрерывно дифференцируема на отрезке $[0,1]$ и по теореме Лагранжа существует точка $\tau \in(0,1)$, для которой $g(1)-g(0)=g^{\prime}(\tau)$. После несложного подсчета будем иметь

$$
\left|\left(|\xi|^{p-2} \xi-|\zeta|^{p-2} \zeta\right) \cdot \eta\right| \leqslant C(n, p)|\xi-\zeta||\zeta+\tau(\xi-\zeta)|^{p-2}|\eta| .
$$

Если $p>2$, то в силу $(3.9)$

$$
\left|\left(|\xi|^{p-2} \xi-|\zeta|^{p-2} \zeta\right) \cdot \eta\right| \leqslant|\xi-\zeta||\zeta|^{p-2}|\eta|
$$

и (3.8) следует из неравенства Юнга. Если же $1<p_{1} \leqslant p<2$, то $p>1+\delta$ для $\delta=\left(p_{1}-1\right) / 2$ и с учетом соотношений $(3.9)-(3.11)$ придем к оценке

$$
\left|\left(|\xi|^{p-2} \xi-|\zeta|^{p-2} \zeta\right) \cdot \eta\right| \leqslant|\xi-\zeta|^{\delta}|\zeta|^{p-1-\delta}|\eta|,
$$

из которой (3.8) вновь вытекает по неравенству Юнга. Предложение доказано.

Прежде чем переходить к изучению вариационных задач, обозначим через $d$ нижнюю грань функционала в (1.7) и приведем одно вспомогательное утверждение для минимизируюшей последовательности $\left\{w_{j}\right\} \in W_{0}(D)$, на которой $F\left(w_{j}+h\right) \rightarrow d$ при $j \rightarrow \infty$.

ПРЕДЛОЖЕНИЕ 3.3. Пусть $\left\{w_{j}\right\}$ - минимизирующая последовательность задачи (1.7) и $d_{0}$ - некоторое положительное число. Тогда для любого $\varepsilon>0$ найдется такое $N$, что при $j>N$ для любой функиии $\psi \in W_{0}(D)$, удовлетворяющей условию $F(\psi)<d_{0}$, выполнено неравенство

$$
\left.\left|\int_{D}\right| \nabla\left(w_{j}+h\right)\right|^{p(x)-2} \nabla\left(w_{j}+h\right) \cdot \nabla \psi d x \mid<\varepsilon .
$$


ДокАЗАТЕЛЬСТво. Пусть $v_{j}=w_{j}+t \psi$, где $t \neq 0,|t| \leqslant 1,-$ некоторое число, которое подберем позже. Так как $F\left(v_{j}+h\right) \geqslant d$, то $F\left(w_{j}+h\right)-F\left(v_{j}+h\right) \leqslant$ $F\left(w_{j}+h\right)-d$, и, полагая $z_{j}=w_{j}+h$, в силу неравенства (3.1) имеем

$$
-t \int_{D}\left|\nabla\left(z_{j}+t \psi\right)\right|^{p(x)-2} \nabla\left(z_{j}+t \psi\right) \cdot \nabla \psi d x \leqslant F\left(z_{j}\right)-d .
$$

Выбирая знак $t$ противоположным знаку интеграла в (3.12), после простого преобразования получим оценку

$$
\begin{aligned}
& \left.\left|\int_{D}\right| \nabla z_{j}\right|^{p(x)-2} \nabla z_{j} \cdot \nabla \psi d x \mid \\
& \quad \leqslant \int_{D}\left|\left(\left|\nabla\left(z_{j}+t \psi\right)\right|^{p(x)-2} \nabla\left(z_{j}+t \psi\right)-\left|\nabla z_{j}\right|^{p(x)-2} \nabla z_{j}\right) \cdot \nabla \psi\right| d x+\frac{F\left(z_{j}\right)-d}{|t|} .
\end{aligned}
$$

Пусть $d_{1}$ - положительная постоянная, оценивающая сверху сходящуюся последовательность $\left\{F\left(z_{j}\right)\right\}$. Из оценки $(3.4)$ и неравенства $F(\psi)<d_{0}$ вытекает, что

$\int_{D}\left|\left(\left|\nabla\left(z_{j}+t \psi\right)\right|^{p(x)-2} \nabla\left(z_{j}+t \psi\right)-\left|\nabla z_{j}\right|^{p(x)-2} \nabla z_{j}\right) \cdot \nabla \psi\right| d x \leqslant C(n, p)|t|^{\delta}\left(d_{0}+d_{1}\right)$.

Выберем теперь $t$ из равенства $C(n, p)|t|^{\delta}\left(d_{1}+d_{0}\right)=\varepsilon / 2$, а после этого найдем такое $N$, чтобы при $j>N$ было справедливо

$$
\frac{F\left(z_{j}\right)-d}{|t|}<\frac{\varepsilon}{2}
$$

Предложение доказано.

Результат предложения 3.3 остается в силе и по отношению к задаче (1.8). В этом случае неравенство (3.12) имеет место на допустимых функциях $\psi \in$ $C_{0}^{\infty}(D)$.

Утверждение, аналогичное предложению 3.3 , имеет место и для минимизирующей последовательности $\left\{u_{j}\right\} \in C_{0}^{\infty}(D)$ вариационной задачи

$$
\inf _{u \in C_{0}^{\infty}(D)} F_{\mu}(u), \quad F_{\mu}(u)=\int_{D} \frac{|\nabla u|^{p(x)}}{p(x)} d x-\int_{D} u d \mu
$$

в которой мера $\mu$ удовлетворяет условию (1.10). Отметим, что в силу этого условия последовательность значений функционалов $\left\{F_{\mu}\left(u_{j}\right)\right\}$ имеет конечньй предел.

ПРЕДЛОЖЕНИЕ 3.4. Пусть $\left\{u_{j}\right\}$ - минимизирующая последовательность задачи (3.13) и $d_{0}$ - некоторое положительное число. Тогда для любого $\varepsilon>0$ найдется такое $N$, что при $j>N$ для любой функиии $\psi \in C_{0}^{\infty}(D)$, удовлетворяющей условию $F(\psi)<d_{0}$, выполнено неравенство

$$
\left.\left|\int_{D}\right| \nabla u_{j}\right|^{p(x)-2} \nabla u_{j} \cdot \nabla \psi d x-\int_{D} \psi d \mu \mid<\varepsilon .
$$


ДокАЗАТЕльСтво.Для доказательства повторим рассуждения, использованные при выводе неравенства (3.12).

2. Разрешимость вариационных задач. Разрешимость вариационных задач $(1.7),(1.8)$ мы установим при одном только предположении (1.2) относительно функции $p(x)$.

ТЕОРема 3.1. Вариационные задачи (1.7) и (1.8) однозначно разрешимы в классах $W_{0}(D)$ и $H_{0}(D)$ соответственно.

ДокАЗАТЕльство. Рассмотрим вариационную задачу (1.7) и покажем, что минимизирующая последовательность $\left\{w_{j}\right\}$ фундаментальна в $W_{0}(D)$. В силу неравенств (3.2), (3.3) имеем

$$
\begin{aligned}
& \int_{E}\left|\nabla\left(w_{i}-w_{j}\right)\right|^{p(x)} d x+\int_{D \backslash E} \frac{\left|\nabla\left(w_{i}-w_{j}\right)\right|^{2}}{\left(\left|\nabla\left(w_{i}+h\right)\right|+\left|\nabla\left(w_{j}+h\right)\right|+1\right)^{2-p(x)}} d x \\
& \leqslant C(p)\left(\left.\left|\int_{D}\right| \nabla\left(w_{j}+h\right)\right|^{p(x)-2} \nabla\left(w_{j}+h\right) \cdot \nabla\left(w_{j}-w_{i}\right) d x \mid\right. \\
&\left.+\left|F\left(w_{i}+h\right)-F\left(w_{j}+h\right)\right|\right),
\end{aligned}
$$

где $E=\{x: x \in D, p(x) \geqslant 2\}$. Зададим $\varepsilon>0$. Пользуясь предложением 3.3 и свойством минимизирующей последовательности, найдем такое $N$, что при $i>N$ и $j>N$ выражение в правой части не превосходит $\varepsilon$, т. е.

$$
\int_{E}\left|\nabla\left(w_{i}-w_{j}\right)\right|^{p(x)} d x+\int_{D \backslash E} \frac{\left|\nabla\left(w_{i}-w_{j}\right)\right|^{2}}{\left(\left|\nabla\left(w_{i}+h\right)\right|+\left|\nabla\left(w_{j}+h\right)\right|+1\right)^{2-p(x)}} d x \leqslant \varepsilon .
$$

Поскольку

$$
\int_{E}\left|\nabla\left(w_{i}-w_{j}\right)\right|^{p(x)} d x \leqslant \varepsilon
$$

остается получить аналогичную оценку на множестве $D \backslash E$. Для этого разобьем $D \backslash E$ на множества

$$
E_{i, j}^{1}=\left\{x: x \in D,\left|\nabla\left(w_{i}-w_{j}\right)\right|^{2-p(x)} \leqslant \varepsilon^{1 / 2}\left(\left|\nabla\left(w_{i}+h\right)\right|+\left|\nabla\left(w_{j}+h\right)\right|+1\right)^{2-p(x)}\right\}
$$

и

$$
E_{i, j}^{2}=\left\{x: x \in D,\left|\nabla\left(w_{i}-w_{j}\right)\right|^{2-p(x)}>\varepsilon^{1 / 2}\left(\left|\nabla\left(w_{i}+h\right)\right|+\left|\nabla\left(w_{j}+h\right)\right|+1\right)^{2-p(x)}\right\} .
$$

Ясно, что

$$
\int_{E_{i, j}^{1}}\left|\nabla\left(w_{i}-w_{j}\right)\right|^{p(x)} d x \leqslant C(p) \varepsilon^{1 / 2}\left(F\left(w_{i}+h\right)+F\left(w_{j}+h\right)+1\right) \leqslant C \varepsilon^{1 / 2},
$$

а в силу (3.15) имеем

$$
\int_{E_{i, j}^{2}}\left|\nabla\left(w_{i}-w_{j}\right)\right|^{p(x)} d x<\varepsilon^{1 / 2}
$$


Таким образом, последовательность $\left\{w_{j}\right\}$ фундаментальна в $W_{0}(D)$ и из леммы 2.4 следует существование предельной функции $w \in W_{0}(D)$. Согласно предложению 2.2

$$
\lim _{j \rightarrow \infty} \int_{D} \frac{\left|\nabla w_{j}\right|^{p(x)}}{p(x)} d x=\int_{D} \frac{|\nabla w|^{p(x)}}{p(x)} d x .
$$

Поэтому функция $w(x)$ является решением вариационной задачи (1.7) и из строгой выпуклости функционала следует, что это решение единственно. Аналогичными рассуждениями доказьвается и однозначная разрешимость задачи (1.8). Отличие заключается лишь в том, что минимизируюшая последовательность состоит из функций $C_{0}^{\infty}(D)$. Теорема доказана.

3. Разрешимость задачи Дирихле в классах $W(D)$ и $H(D)$. Сначала покажем однозначную разрешимость задач (1.5) и (1.6).

Теорема 3.2. Каждая из задач Дирихле (1.5), (1.6) однозначно разрешима. Если выполнено условие (1.12), то рассматриваемые задачи совпадают и существует единственное решение из класса $W(D)$.

ДокаЗАтельство. Рассмотрим задачу (1.5). Нетрудно видеть, что доказательство ее разрешимости сводится к нахождению функции $w \in W_{0}(D)$, удовлетворяющей интегральному тождеству

$$
\int_{D}|\nabla(w+h)|^{p(x)-2} \nabla(w+h) \cdot \nabla \psi d x=0
$$

на пробных функциях $\psi \in W_{0}(D)$. Искомым решением (3.16) является минимизант задачи (1.7). Действительно, в силу линейности класса $W_{0}(D)$ минимизант $w(x)$ удовлетворяет неравенству

$$
\int_{D} \frac{|z+t \nabla \psi|^{p(x)}-|z|^{p(x)}}{t p(x)} d x \geqslant 0 \quad \forall \psi \in W_{0}(D), \quad t>0
$$

где полагается $z=\nabla(w+h)$. Очевидно, что

$$
\lim _{t \rightarrow+0} \frac{|z+t \nabla \psi|^{p(x)}-|z|^{p(x)}}{t p(x)}=|z|^{p(x)-2} z \cdot \nabla \psi
$$

почти всюду в $D$, и необходимо проверить, что здесь имеет место сходимость в $L_{1}(D)$. Если $t \in(0,1)$, то в силу неравенства $(2.4)$ имеем

$$
\begin{aligned}
\left|\frac{|z+t \nabla \psi|^{p}-|z|^{p}}{t p}\right| & \leqslant\left(|z+t \nabla \psi|^{p-1}+|z|^{p-1}\right)|\nabla \psi| \\
& \leqslant C(p)\left(|z|^{p-1}+|\nabla \psi|^{p-1}\right)|\nabla \psi| \leqslant C(p)\left(|z|^{p}+|\nabla \psi|^{p}\right) \in L_{1}(D),
\end{aligned}
$$

и по теореме Лебега в (3.17) можно перейти к пределу при $t \rightarrow+0$. Отсюда вытекает, что интеграл в (3.16) неотрицателен для любой функции $\psi \in W_{0}(D)$, а потому равен нулю и тождество (3.16) установлено. Осталось показать единственность. 
Пусть $u(x)$ и $v(x)$ - решения задачи (1.5). Из интегральных тождеств (1.4) для $u(x)$ и $v(x)$ с пробной функцией $\psi=(u-v) \in W_{0}(D)$ следует, что

$$
\int_{D}\left(|\nabla u|^{p(x)-2} \nabla u-|\nabla v|^{p(x)-2} \nabla v\right) \cdot \nabla(u-v) d x=0 .
$$

Поскольку

$$
\left(|\xi|^{p(x)} \xi-|\zeta|^{p(x)} \zeta\right) \cdot(\xi-\zeta)>0 \quad \forall \xi, \zeta \in \mathbb{R}^{n}, \quad \xi \neq \zeta,
$$

то предыдушее тождество влечет совпадение $u(x)$ и $v(x)$ почти всюду в $D$. Точно так же устанавливается и однозначная разрешимость задачи (1.6). Если выполнено условие $(1.12)$, то $W(D)=H(D), W_{0}(D)=H_{0}(D)$ и рассматриваемые задачи совпадают. Теорема доказана.

ТЕОРема 3.3. Если мера $\mu$ удовлетворяет условию (1.10), то задача Дирихле (1.9) однозначно разрешима.

ДокАЗАТЕЛЬСТвО. Пользуясь оценкой (3.14) и повторяя доказательство теоремы 3.1 , нетрудно установить фундаментальность в $W_{0}(D)$ минимизирующей последовательности $\left\{u_{j}\right\} \in C_{0}^{\infty}(D)$ вариационной задачи (3.13). Покажем, что предельная функция $u \in H_{0}(D)$ является решением задачи (1.9). Пусть $\psi \in C_{0}^{\infty}(D)$. В силу неравенства $(3.8)$ и сходимости $u_{j}(x)$ к $u(x)$ в $W_{0}(D)$ имеем

$$
\begin{aligned}
& \left|\int_{D}\left(\left|\nabla u_{j}\right|^{p(x)-2} \nabla u_{j}-|\nabla u|^{p(x)-2} \nabla u\right) \cdot \nabla \psi d x\right| \\
& \quad \leqslant C(n, p, \varepsilon) \int_{D}\left|\nabla\left(u-u_{j}\right)\right|^{p(x)} d x+\varepsilon \int_{D}\left(|\nabla u|^{p(x)}+|\nabla \psi|^{p(x)}\right) d x \\
& \quad \leqslant \varepsilon+\varepsilon \int_{D}\left(|\nabla u|^{p(x)}+|\nabla \psi|^{p(x)}\right) d x \text { при } j>j_{0}(\varepsilon),
\end{aligned}
$$

и из оценки $(3.14)$ при $j>j_{1}(n, p, \psi, \varepsilon)$ получим

$$
\left.\left|\int_{D}\right| \nabla u\right|^{p(x)-2} \nabla u \cdot \nabla \psi d x-\int_{D} \psi d \mu \mid \leqslant 2 \varepsilon+\varepsilon \int_{D}\left(|\nabla u|^{p(x)}+|\nabla \psi|^{p(x)}\right) d x .
$$

Отсюда ввиду произвольности $\varepsilon$ следует, что функция $u \in H_{0}(D)$ удовлетворяет интегральному тождеству (1.11), а значит, является решением задачи (1.9).

Докажем единственность. Пусть $u, v \in H_{0}(D)$ - решения задачи (1.9), $w=$ $u-v$ и $\left\{\psi_{j}\right\} \in C_{0}^{\infty}(D)$ - последовательность, сходящаяся к $w$ в $W_{0}(D)$. Согласно интегральным тождествам для $u(x)$ и $v(x)$ имеем

$$
\int_{D}\left(|\nabla u|^{p(x)-2} \nabla u-|\nabla v|^{p(x)-2} \nabla v\right) \cdot \nabla \psi_{j} d x=0 .
$$

Переходя к пределу при $j \rightarrow \infty$, придем к равенству (3.18), из которого вытекает, что $u(x)=v(x)$ почти всюду в $D$. Теорема доказана.

Для $W$ - и $H$-решений уравнения (1.1) имеет место принцип максимума. 
ПРИНЦИП МАКСИМУМА. Если функиии $u, v \in W(D)(u, v \in H(D))$ являются $W$-решениями (H-решениями) уравнения (1.1) $и u \geqslant v$ на $\partial D$ в смысле $W(D)$ (соответственно, в смысле $H(D)$ ), то $u(x) \geqslant v(x)$ почти всюду в $D$.

ДокАЗАТЕЛЬСТво. Пусть $u, v \in W(D)$. Из интегральных тождеств (1.4) для решений $u(x)$ и $v(x)$ с пробной функцией $\psi=(u-v)^{-} \in W_{0}(D)$ следует, что

$$
\int_{E}\left(|\nabla u|^{p(x)-2} \nabla u-|\nabla v|^{p(x)-2} \nabla v\right) \cdot \nabla \psi d x=0,
$$

где $E=\{x: u(x) \leqslant v(x)\}$ и $u(x)=v(x)$ почти всюду в $D$ в силу неравенства (3.19). Если $u, v \in H(D)$, то доказательство ничем не отличается от приведенного выше.

Принцип максимума справедлив при одном только предположении (1.2) относительно измеримого в $D$ показателя суммируемости $p(x)$. Если выполнено условие (1.12), то, как отмечалось вьше, решения уравнения (1.1) из класса $W_{\text {loc }}(D)$ гёльдеровы в $D$ и в формулировке принципа максимума $u(x) \geqslant v(x)$ всюду в $D$.

\section{§4. Разрешимость обобщенной задачи Дирихле}

Для доказательства разрешимости обобщенной задачи Дирихле используется свойство повьшенной суммируемости градиента ограниченного в области $D$ решения из класса $W_{\text {loc }}(D)$. Ключевую роль в доказательстве этого свойства играют локальное обобщение леммы Геринга [35], полученное в [36], и оценка градиента ограниченного решения во внутренних шарах области $D$, доказанная в работе [8].

Ниже $u(x)$ - ограниченное решение уравнения (1.1) из класса $W_{\mathrm{loc}}(D), \quad M=$ $\sup _{D}|u(x)|$,

$$
f_{B_{r}} \varphi(x) d x=\frac{1}{\left|B_{r}\right|} \int_{B_{r}} \varphi(x) d x-\text { среднее функции по } B_{r} \text {. }
$$

В следуюшей лемме полагается $t=\min \left(p_{1},(n+1) / n\right)$, где $p_{1}>1$ - постоянная из условия (1.2).

ЛЕмма 4.1 [8]. Если выполнены условия (1.2) и (1.12), то существует положительная постоянная $r_{0}(n, p)<1 / 4$ такая, что для любого решения $u(x)$ уравнения (1.1) из класса $W_{\mathrm{loc}}(D) \cap L_{\infty}(D)$ в произвольном шаре $B_{2 r} \subset D$ при $r \leqslant r_{0}(n, p)$ имеет место оченка

$$
f_{B_{r / 2}}|\nabla u|^{p(x)} d x \leqslant C(n, p, M)\left(\left(f_{B_{r}}|\nabla u|^{p(x) / t} d x\right)^{t}+1\right) .
$$

Сформулируем теперь свойство повьшенной суммируемости градиента решения уравнения (1.1).

ЛЕмма 4.2. Если выполнены условия (1.2) и (1.12), то существует положительная постоянная $\delta=\delta(n, p, M)$ такая, что для любого решения $u \in W_{\mathrm{loc}}(D) \cap L_{\infty}(D)$ уравнения (1.1) вьполнено $|\nabla u|^{p(x)} \in L_{1+\delta, \operatorname{loc}}(D)$ 
и в каждой строго внутренней подобласти $D^{\prime}$ области $D$ имеет место оченка

$$
\int_{D^{\prime}}|\nabla u|^{(1+\delta) p(x)} d x \leqslant C,
$$

в которой постоянная $C$ зависит только от размерности пространства $n$, показателя суммируемости $p(x)$, диаметра области $D$, расстояния от $D^{\prime}$ до гранищы $\partial D$ области $D$ и точной верхней грани $M$ модуля решения $u(x)$.

ДокАЗАТЕЛЬСтво. Пусть $\operatorname{dist}\left(D^{\prime}, \partial D\right)$ - расстояние от подобласти $D^{\prime}$ до границы области $D, \rho \leqslant \min \left(\operatorname{dist}\left(D^{\prime}, \partial D\right), r\right)$, где $r_{0}$ - постоянная из условия леммы 4.1, и $B_{\rho / 2}^{x_{0}}$ - шар с центром на $\bar{D}^{\prime}$. Воспользуемся оценкой (4.2) в произвольном шаре $B_{r} \subset B_{\rho / 2}^{x_{0}}$. Из этой оценки и полученного в [36] результата, содержашего локальное обобщение леммы Геринга, следует существование положительной постоянной $\delta$, зависяшей только от $n, p$ и $M$, такой, что $|\nabla u|^{p(x)} \in L_{1+\delta, \text { loc }}\left(B_{\rho / 2}^{x_{0}}\right)$ и выполнено неравенство

$$
f_{B_{\rho / 4}^{x_{0}}}|\nabla u|^{(1+\delta) p(x)} d x \leqslant C(n, p, M)\left(\left(\int_{B_{\rho / 2}^{x_{0}}}|\nabla u|^{p(x)} d x\right)^{1+\delta}+1\right) .
$$

Для оценки интеграла в правой части (4.4) выберем из интегрального тождества (1.4) пробную функцию $\psi(x)=u(x) \eta^{s}(x)$, где $s$ - точная верхняя грань $p(x)$ в шаре $B_{\rho}^{x_{0}}, \eta \in C_{0}^{\infty}\left(B_{\rho}^{x_{0}}\right), 0 \leqslant \eta(x) \leqslant 1, \eta(x) \equiv 1$ при $x \in B_{\rho / 2}^{x_{0}}$ и $|\nabla \eta| \leqslant C \rho^{-1}$. В силу интегрального тождества

$$
f_{B_{\rho}^{x_{0}}}|\nabla u|^{p(x)} \eta^{s} d x \leqslant C(p) \int_{B_{\rho}^{x_{0}}}|\nabla u|^{p(x)-1}|u| \eta^{s-1} \rho^{-1} d x .
$$

Применяя неравенство Юнга

$$
|\nabla u|^{p(x)-1}|u| \eta^{s-1} \rho^{-1} \leqslant \varepsilon|\nabla u|^{p(x)} \eta^{s}+C(\varepsilon)|u|^{p(x)} \eta^{s-p(x)} \rho^{-p(x)},
$$

после соответствуюшего выбора $\varepsilon$ придем к соотношению

$$
\int_{B_{\rho / 2}^{x_{0}}}|\nabla u|^{p(x)} d x \leqslant C(p) f_{B_{\rho}^{x_{0}}}|u|^{p(x)} \rho^{-p(x)} d x \leqslant C(p) \rho^{-p_{2}}\left(M^{p_{2}}+1\right),
$$

в котором $p_{2}$ - постоянная из условия (1.2). Теперь неравенство (4.4) примет вид

$$
\int_{B_{\rho / 4}^{x_{0}}}|\nabla u|^{(1+\delta) p(x)} d x \leqslant C(n, p, \rho, M)
$$

Покроем $D^{\prime}$ конечным числом шаров $B_{\rho / 4}^{x_{i}}$ с центрами на $\bar{D}^{\prime}$. Кратность такого покрытия можно считать зависящей только от размерности пространства $n$, так что количество покрывающих $D^{\prime}$ шаров не превосходит $C(n) \cdot \operatorname{diam}(D) \cdot \rho^{-n}$, где $\operatorname{diam}(D)$ - диаметр области $D$. Суммируя неравенства (4.5) по всем шарам покрытия, придем к требуемому соотношению (4.3). Лемма доказана.

Ниже $C^{\alpha}(D)$ означает пространство гёльдеровых в $D$ функций с показателем Гёльдера $\alpha \in(0,1)$. 
ТЕОРема 4.1. Если выполнены условия (1.2) и (1.12), то обобщенное решение $u_{f}(x)$ задачи (1.15) существует, единственно и принадлежит классу $W_{\mathrm{loc}}(D)$.

ДокАЗАТЕЛЬСТво. Пусть $f \in C\left(\mathbb{R}^{n}\right)$ - непрерьвное продолжение в $\mathbb{R}^{n}$ граничной функции задачи (1.15), $\left\{f_{k}\right\} \in C^{\infty}\left(\mathbb{R}^{n}\right)$ - последовательность, сходящаяся к $f$ равномерно на $\bar{D}$, и $\left\{u_{k}\right\}$ - последовательность решений задач Дирихле (1.16). По теореме 3.2 задачи (1.16) однозначно разрешимы в классе $W(D)$. Из принципа максимума следует равномерная сходимость $\left\{u_{k}\right\}$ на компактных подмножествах $D$ и независимость предельной функции $u_{f}(x)$ от способа продолжения и аппроксимации граничной функции $f(x)$. Поскольку решения задач (1.16) равномерно ограничены в $D$, то (см. [16]) существует показатель $\alpha \in(0,1)$ такой, что последовательность $\left\{u_{k}\right\}$ компактна в $C^{\alpha}\left(D^{\prime}\right)$ в каждой строго внутренней подобласти $D^{\prime}$ области $D$. Таким образом, $u_{f} \in C^{\alpha}(D)$.

Покажем, что $u_{f}(x)$ принадлежит классу $W_{\text {loc }}(D)$ и удовлетворяет интегральному тождеству (1.4) на пробных функциях $\psi \in C_{0}^{\infty}(D)$. Обозначим через $M$ постоянную, равномерно ограничивающую сверху точные верхние грани модулей решений $u_{k}(x)$. Согласно лемме 4.2 существует постоянная $\delta(n, p, M)>0$ такая, что $\left|\nabla u_{k}\right|^{p(x)} \in L_{1+\delta \text {, lос }}(D)$. Пусть $\bar{B}_{r} \subset D$ - шар, радиус $r$ которого столь мал, что

$$
(1+\delta) p(x)>q=\left(1+\frac{\delta}{2}\right) s, \quad x \in B_{r}, \quad s=\sup _{B_{r}} p(x) .
$$

Из оценки (4.3) и равномерной ограниченности решений $u_{k}(x)$ вытекает слабая компактность последовательности $\left\{u_{k}\right\}$ в соболевском пространстве $W_{q}^{1}\left(B_{r}\right)$. Поскольку $u_{k}(x)$ сходится к $u_{f}(x)$ поточечно в $D$, то, как хорошо известно (см., например, [21, теорема 1.32]),

$$
u_{k} \rightarrow u_{f} \text { слабо в } W_{q}^{1}\left(B_{r}\right) .
$$

При этом согласно (4.6) имеем

$$
\left|\nabla u_{f}\right|^{p(x)-1} \in L_{q^{\prime}}\left(B_{r}\right), \quad q^{\prime}=\frac{q}{q-1} .
$$

Будем пользоваться равенствами

$$
\lim _{k \rightarrow \infty} \int_{B_{r}}\left|\nabla u_{f}\right|^{p(x)-2} \nabla u_{f} \cdot\left(\nabla\left(u_{f} \eta\right)-\nabla\left(u_{k} \eta\right)\right) d x=0
$$

и

$$
\lim _{k \rightarrow \infty} \int_{B_{r}}\left(\left|\nabla u_{f}\right|^{p(x)-2} \nabla u_{f}-\left|\nabla u_{k}\right|^{p(x)-2} \nabla u_{k}\right) \cdot \nabla \eta\left(u_{f}-u_{k}\right) d x=0,
$$

в которых $\eta \in C_{0}^{\infty}\left(B_{r}\right)$. Данные равенства являются простым следствием равномерной сходимости $u_{k}(x)$ к $u_{f}(x)$ на компактных подмножествах $D$, слабой сходимости (4.7) и соотношения (4.8). Поскольку функции $u_{k}(x)$ являются решениями задач Дирихле (1.16), то из интегрального тождества с пробной функцией $\psi(x)=\eta(x) u_{k}(x)$ вытекает, что

$$
\begin{aligned}
\int_{B_{r}} \mid \nabla & \left.u_{f}\right|^{p(x)-2} \nabla u_{f} \cdot\left(\nabla\left(u_{f} \eta\right)-\nabla\left(u_{k} \eta\right)\right) d x \\
& =\int_{B_{r}}\left(\left|\nabla u_{f}\right|^{p(x)-2} \nabla u_{f}-\left|\nabla u_{k}\right|^{p(x)-2} \nabla u_{k}\right) \cdot\left(\nabla\left(u_{f} \eta\right)-\nabla\left(u_{k} \eta\right)\right) d x .
\end{aligned}
$$


Переписав последнее равенство в виде

$$
\begin{aligned}
\int_{B_{r}}\left(\left|\nabla u_{f}\right|^{p(x)-2} \nabla u_{f}-\left|\nabla u_{k}\right|^{p(x)-2} \nabla u_{k}\right) \cdot\left(\nabla u_{f}-\nabla u_{k}\right) \eta d x \\
=\int_{B_{r}}\left|\nabla u_{f}\right|^{p(x)-2} \nabla u_{f} \cdot\left(\nabla\left(u_{f} \eta\right)-\nabla\left(u_{k} \eta\right)\right) d x \\
\quad-\int_{B_{r}}\left(\left|\nabla u_{f}\right|^{p(x)-2} \nabla u_{f}-\left|\nabla u_{k}\right|^{p(x)-2} \nabla u_{k}\right) \cdot \nabla \eta\left(u_{f}-u_{k}\right) d x
\end{aligned}
$$

в силу (4.9) и (4.10) имеем

$$
\lim _{k \rightarrow \infty} \int_{B_{r}}\left(\left|\nabla u_{f}\right|^{p(x)-2} \nabla u_{f}-\left|\nabla u_{k}\right|^{p(x)-2} \nabla u_{k}\right) \cdot\left(\nabla u_{f}-\nabla u_{k}\right) \eta d x=0 .
$$

Рассмотрим выражение (4.11) на неотрицательных функциях $\eta(x)$. Из неравенства (3.19) и произвольности $\eta \in C_{0}^{\infty}\left(B_{r}\right)$ следует существование подпоследовательности последовательности $\left\{u_{k}\right\}$, за которой сохраним прежнее обозначение, такой, что

$$
\lim _{k \rightarrow \infty}\left(\left|\nabla u_{f}(x)\right|^{p(x)-2} \nabla u_{f}(x)-\left|\nabla u_{k}(x)\right|^{p(x)-2} \nabla u_{k}(x)\right) \cdot\left(\nabla u_{f}(x)-\nabla u_{k}(x)\right)=0
$$

для почти всех $x \in B_{r}$.

Пусть $x \in B_{r}$ - точка, в которой выполнено равенство $(4.12), \xi=\nabla u_{f}(x)$ и $\xi^{\star}-$ какая-либо предельная точка последовательности $\left\{\nabla u_{k}(x)\right\}$. Если $\left|\xi^{\star}\right|=\infty$, то $\left|\nabla u_{k}(x)\right| \rightarrow \infty$ по некоторой подпоследовательности номеров $k$ и неравенство

$$
\begin{aligned}
& \left(\left|\nabla u_{f}(x)\right|^{p(x)-2} \nabla u_{f}(x)-\left|\nabla u_{k}(x)\right|^{p(x)-2} \nabla u_{k}(x)\right) \cdot\left(\nabla u_{f}(x)-\nabla u_{k}(x)\right) \\
& \geqslant\left|\nabla u_{k}(x)\right|^{p(x)}-\left|\nabla u_{f}(x)\right|\left|\nabla u_{k}(x)\right|^{p(x)-1}-\left|\nabla u_{f}(x)\right|^{p(x)-1}\left|\nabla u_{k}(x)\right|
\end{aligned}
$$

ведет к противоречию с (4.12). Поэтому $\left|\xi^{\star}\right|<\infty$, а так как

$$
\sum_{i=1}^{n}\left(|\xi|^{p(x)-2} \xi_{i}-\left|\xi^{\star}\right|^{p(x)-2} \xi_{i}^{\star}\right)\left(\xi_{i}-\xi_{i}^{\star}\right)=0
$$

то $\xi^{\star}=\xi=\nabla u_{f}(x)$ и

$$
\nabla u_{k} \rightarrow \nabla u_{f} \quad \text { почти всюду в } B_{r} .
$$

Из (4.13) следует, что в интегральном тождестве

$$
\int_{B_{r}}\left|\nabla u_{k}\right|^{p(x)-2} \nabla u_{k} \cdot \nabla \eta d x=0, \quad \eta \in C_{0}^{\infty}\left(B_{r}\right)
$$

можно перейти к пределу под знаком интеграла. Действительно, слабая компактность последовательности $\left\{u_{k}\right\}$ в $W_{q}^{1}\left(B_{r}\right)$ (см. (4.6)) влечет равностепенную интегрируемость последовательности $\left\{\left|\nabla u_{k}\right|^{p(x)-1}\right\}$, и по теореме Лебега

$$
\int_{B_{r}}\left|\nabla u_{f}\right|^{p(x)-2} \nabla u_{f} \cdot \nabla \eta d x=0 \quad \forall \eta \in C_{0}^{\infty}\left(B_{r}\right) .
$$


Осталось показать, что

$$
\int_{D}\left|\nabla u_{f}\right|^{p(x)-2} \nabla u_{f} \cdot \nabla \psi d x=0 \quad \forall \psi \in C_{0}^{\infty}(D) .
$$

Пусть $\left\{\mathcal{B}_{r_{k}}\right\}$ - локально конечное покрытие области $D$ открытыми шарами $\overline{\mathcal{B}}_{r_{k}} \subset D$, радиусы $r_{k}$ которых удовлетворяют условию $(4.6),\left\{\eta_{k}\right\}$ - подчиненное этому покрытию разбиение единицы и $\psi_{k}(x)=\psi(x) \cdot \eta_{k}(x)$. Поскольку на носителе $\psi(x)$ в разложении $\psi(x)=\sum \psi_{k}(x)$ участвует только конечное число слагаемых, то, пользуясь тождеством (4.14) на пробных функциях $\psi_{k}(x) \in C_{0}^{\infty}\left(\mathcal{B}_{r_{k}}\right)$, придем к (4.15). Теорема доказана.

\section{§5. Емкость и емкостный потенциал}

1. Емкость. В определении 1.5 емкости компакта $K \subset B_{R}$ относительно шара $B_{R}$ точная нижняя грань функционала в (1.17) берется по множеству функций

$$
\mathcal{N}(K)=\left\{\varphi \in C_{0}^{\infty}\left(B_{R}\right): \varphi \geqslant 1 \text { на } K\right\}
$$

Сначала покажем, что

$$
C_{p}\left(K, B_{R}\right)=\inf _{\varphi \in \mathcal{M}(K)} F(\varphi), \quad F(\varphi)=\int_{B_{R}} \frac{|\nabla \varphi|^{p(x)}}{p(x)} d x
$$

где

$$
\mathcal{M}(K)=\left\{\varphi \in C_{0}^{\infty}\left(B_{R}\right): \varphi=1 \text { в окрестности } K\right\} .
$$

Действительно, так как $\mathcal{M}(K) \subset \mathcal{N}(K)$, то точную нижнюю грань в (5.1) достаточно оценить емкостью $C_{p}\left(K, B_{R}\right)$ только сверху. Пусть $\varphi \in \mathcal{N}(K)$ - такая функция, что $F(\varphi)<C_{p}\left(K, B_{R}\right)+\varepsilon$ для заданного $\varepsilon>0$. Обозначим через $\left\{\eta_{j}\right\}$, $j=1,2, \ldots$, последовательность функций из $C^{\infty}\left(\mathbb{R}^{1}\right)$, удовлетворяющую условиям: $0 \leqslant \eta_{j}(t) \leqslant 1+j^{-1}, \eta_{j}(t)=0$ в окрестности $(-\infty, 0], \eta_{j}(t)=1$ в окрестности $[1, \infty)$ и $0 \leqslant \eta_{j}(t) \leqslant 1$ при всех $j$. Поскольку $\eta_{j}(\varphi(x)) \in \mathcal{M}(K)$, то

$$
\inf _{\varphi \in \mathcal{M}(K)} F(\varphi) \leqslant \int_{B_{R}} \frac{\left|\eta_{j}^{\prime}(\varphi(x))\right|^{p(x)}|\nabla \varphi|^{p(x)}}{p(x)} d x .
$$

Переходя к пределу при $j \rightarrow \infty$, получим

$$
\inf _{\varphi \in \mathcal{M}(K)} F(\varphi) \leqslant C_{p}\left(K, B_{R}\right)+\varepsilon
$$

что и доказывает (5.1).

Из определения емкости вытекают следующие свойства:

i) для любых компактов $K_{1} \subset K_{2}$ вьполнено $C_{p}\left(K_{1}, B_{R}\right) \leqslant C_{p}\left(K_{2}, B_{R}\right)$;

ii) емкость компакта $K \subset B_{R}$ не возрастает при расширении шара $B_{R}$. 
2. Оценки емкости шара. В случае $p(x) \equiv p_{0}=$ const свойства емкости исследованы в [40]. В частности,

$$
C_{p_{0}}\left(\bar{B}_{r}, B_{R}\right)= \begin{cases}\omega_{n}\left(\frac{\left|n-p_{0}\right|}{p_{0}-1}\right)^{p_{0}-1} \mid r^{\frac{p_{0}-n}{p_{0}-1}}-R^{\left.\frac{p_{0}-n}{p_{0}-1}\right|^{1-p_{0}},}, & \text { если } p_{0} \neq n, \\ \omega_{n}\left(\ln \frac{R}{r}\right)^{1-n}, & \text { если } p_{0}=n,\end{cases}
$$

где $\omega_{n}-$ плошадь единичной сферы в $\mathbb{R}^{n}$.

Приведем оценки емкости замкнутого шара $\bar{B}_{r}=\bar{B}_{r}^{x_{0}}$ относительно концентрического ему шара $B_{R}$ достаточно малого радиуса $R<R_{0}(p)$ для показателя суммируемости $p(x)$, удовлетворяющего условию (1.12). Положим $p_{0}=p\left(x_{0}\right)$ и, не ограничивая общности, будем считать, что $x_{0}$ совпадает с началом координат.

ПРЕДЛОЖЕНИЕ 5.1. Если $r \leqslant R / 2$, mo

$$
C_{p}\left(\bar{B}_{r}, B_{R}\right) \leqslant\left\{\begin{array}{lll}
C(n, p) r^{n-p_{0}}, & \text { ecлu } & p_{0}<n \\
C(n, p) R^{n-p_{0}}, & \text { ecлu } & p_{0}>n \\
C(n, p)\left(\ln \frac{R}{r}\right)^{1-n}, & \text { ecлu } & p_{0}=n .
\end{array}\right.
$$

ДокаЗАТЕЛЬСтво. В случае $p_{0}<n$ рассмотрим срезающую функцию $\varphi \in$ $C_{0}^{\infty}\left(B_{2 r}\right), \varphi=1$ в окрестности $B_{r},|\nabla \varphi| \leqslant C r^{-1}$. По определению емкости и условию (1.12) имеем

$$
C_{p}\left(\bar{B}_{r}, B_{R}\right) \leqslant C(p) \int_{B_{R}}|\nabla \varphi|^{p(x)} d x \leqslant C(p) \int_{B_{2 r}} r^{-p(x)} d x \leqslant C(n, p) r^{n-p_{0}},
$$

что доказывает первую оценку в (5.4).

Если $p_{0}>n$, то возьмем срезающую функцию $\varphi \in C_{0}^{\infty}\left(B_{R}\right), \varphi=1$ в окрестности $B_{R / 2},|\nabla \varphi| \leqslant C R^{-1}$. Действуя так же, как и выше, получим вторую оценку В $(5.4)$.

В случае $p_{0}=n$ определим на множестве $B_{R} \backslash B_{r}$ функцию $v(x)=(\ln (R / r))^{-1} \times$ $\ln (R /|x|)$ и обозначим через $\eta(t)$ бесконечно дифференцируемую на $[0,1]$ функцию, равную нулю вблизи точки $t=0$, единице вблизи $t=1$ и такую, что $0 \leqslant \eta(t) \leqslant 1$. Пусть $\varphi(x)=\eta(v(x))$ при $x \in B_{R} \backslash B_{r}$ и $\varphi(x)=1$ в $B_{r}$. Ясно, что функция $\varphi(x)$ принадлежит классу допустимых функций, участвующих в определении емкости, и $|\nabla \varphi(x)| \leqslant C(\ln (R / r))^{-1}|x|^{-1}$ на $B_{R} \backslash B_{r}$. Поэтому

$$
C_{p}\left(\bar{B}_{r}, B_{R}\right) \leqslant C(p) \int_{B_{R}}|\nabla \varphi|^{p(x)} d x \leqslant C(p) \int_{B_{R} \backslash B_{r}}\left(\ln \frac{R}{r}\right)^{-p(x)}|x|^{-p(x)} d x .
$$

Пусть радиус $R$ столь мал, что $|p(x)-n| \leqslant 1 / 2$ для всех $x \in B_{R}$. Тогда в силу монотонности функции $\ln (R / t)$ по переменной $t \in[r, R]$ и условия (1.12) имеем

$$
\begin{aligned}
& \left(\ln \frac{R}{r}\right)^{-p(x)}|x|^{-p(x)} \\
& \quad \leqslant C(p)\left(\ln \frac{R}{r}\right)^{1 / 2-n}\left(\ln \frac{R}{|x|}\right)^{-1 / 2}|x|^{-n} \forall x \in B_{R} \backslash B_{r} .
\end{aligned}
$$


Теперь с учетом предыдущей оценки получим

$$
\begin{aligned}
C_{p}\left(\bar{B}_{r}, B_{R}\right) & \leqslant C(p)\left(\ln \frac{R}{r}\right)^{1 / 2-n} \int_{B_{R} \backslash B_{r}}\left(\ln \frac{R}{|x|}\right)^{-1 / 2}|x|^{-n} d x \\
& =C(n, p)\left(\ln \frac{R}{r}\right)^{1-n} .
\end{aligned}
$$

Предложение доказано.

ПРЕДЛОЖЕНИЕ 5.2. Если существуют постоянные $\alpha \geqslant 1 u \beta>0$ такие, что $R^{\alpha} / 4 \leqslant r \leqslant R / 2$ в случае $p_{0}<n$ u $\exp \left(-\beta R^{-1}\right) \leqslant r \leqslant R / 2$ в случае $p_{0}=n$, mo

$$
C_{p}\left(\bar{B}_{r}, B_{R}\right) \geqslant\left\{\begin{array}{lll}
C(n, p, \alpha) r^{n-p_{0}}, & \text { eслu } & p_{0}<n, \\
C(n, p) R^{n-p_{0}}, & \text { ecлu } & p_{0}>n, \\
C(n, p, \beta)\left(\ln \frac{R}{r}\right)^{1-n}, & \text { eслu } & p_{0}=n .
\end{array}\right.
$$

ДокАЗАТЕЛЬСТво. Перейдем к сферической системе координат $(\rho, \omega),|\omega|=1$, и рассмотрим множество функций $\mathcal{M}\left(\bar{B}_{r}\right)$ (см. (5.2)). Ясно, что

$$
\begin{aligned}
C_{p}\left(\bar{B}_{r}, B_{R}\right) & \geqslant \inf _{\varphi \in \mathcal{M}\left(\bar{B}_{r}\right)} \int_{\partial B_{1}} d \omega \int_{r}^{R}\left|\frac{\partial \varphi}{\partial \rho}\right|^{p} \frac{\rho^{n-1}}{p(\rho, \omega)} d \rho \\
& \geqslant \int_{\partial B_{1}} d \omega \inf _{\varphi \in \mathcal{M}\left(\bar{B}_{r}\right)} \int_{r}^{R}\left|\frac{\partial \varphi}{\partial \rho}\right|^{p} \frac{\rho^{n-1}}{p(\rho, \omega)} d \rho .
\end{aligned}
$$

Точная нижняя грань внутреннего интеграла достигается на функции

$$
v(\rho, \omega)=\int_{\rho}^{R} d^{1 /(p-1)} t^{-(n-1) /(p-1)} d t,
$$

где $p=p(t, \omega)$, а неотрицательная, непрерывная на единичной сфере функция $d=$ $d(\omega)$ такова, что

$$
\int_{r}^{R} d^{1 /(p-1)} t^{-(n-1) /(p-1)} d t=1 .
$$

Таким образом,

$$
C_{p}\left(\bar{B}_{r}, B_{R}\right) \geqslant \int_{\partial B_{1}} d \omega \int_{r}^{R} d^{p /(p-1)} t^{-(n-1) /(p-1)} d t .
$$

Если $d \leqslant 1$, то, полагая $s=\max _{\bar{B}_{R}} p(x)$, из неравенства Гёльдера, соотношения (5.6) и условия (1.12) имеем

$$
\begin{aligned}
I & =\int_{r}^{R} d^{p /(p-1)} t^{-(n-1) /(p-1)} d t \geqslant \int_{r}^{R} d^{s /(p-1)} t^{-(n-1) /(p-1)} d t \\
& \geqslant\left(\int_{r}^{R} d^{1 /(p-1)} t^{-(n-1) /(p-1)} d t\right)^{s}\left(\int_{r}^{R} t^{-(n-1) /(p-1)} d t\right)^{1-s} \\
& \geqslant C(p)\left(\int_{r}^{R} t^{-(n-1) /\left(p_{0}-1\right)} d t\right)^{1-p_{0}} .
\end{aligned}
$$


Если $d>1$, то, обозначив $\sigma=\min _{\bar{B}_{R}} p(x)$, точно так же можно показать, что

$$
I \geqslant\left(\int_{r}^{R} t^{-(n-1) /(p-1)} d t\right)^{1-\sigma} \geqslant C(p)\left(\int_{r}^{R} t^{-(n-1) /\left(p_{0}-1\right)} d t\right)^{1-p_{0}} .
$$

Требуемые оценки (5.5) вытекают из (5.7) и двух последних неравенств. Предложение доказано.

Из полученных оценок следует, что если $p_{0}>n$, то емкость точки $\left\{x_{0}\right\}$ относительно шара $B_{R}^{x_{0}}$ положительна и имеют место оценки

$$
C_{1}(n, p) R^{n-p_{0}} \leqslant C_{p}\left(\left\{x_{0}\right\}, B_{R}^{x_{0}}\right) \leqslant C_{2}(n, p) R^{n-p_{0}} .
$$

Если же $p_{0} \leqslant n$, то

$$
C_{p}\left(\bar{B}_{b R}, B_{R}\right) \geqslant C(n, p, b) R^{n-p_{0}} \text { при } 0<b \leqslant \frac{1}{2} .
$$

3. Емкостный потенциал. Рассмотрим вариационную задачу (5.1).

Tеорема 5.1. Существует единственная функиия $и \in H_{0}\left(B_{R}\right)$ такая, ито $u=1$ на $K$ в смислее $H\left(B_{R}\right) u$

$$
C_{p}\left(K, B_{R}\right)=F(u) .
$$

ДокАЗАТЕЛЬСТво. Пусть $\left\{u_{j}\right\} \in C_{0}^{\infty}\left(B_{R}\right)$ - минимизирующая последовательность задачи (5.1). Как и в доказательстве предложения 3.3 , можно показать, что для заданного $\varepsilon>0$ и любой функции $\psi \in C_{0}^{\infty}\left(B_{R}\right)$, равной нулю в окрестности компакта $K$ и удовлетворяюшей условию $F(\psi) \leqslant d_{0}$, при $j>N\left(n, p, d_{0}, \varepsilon\right)$ выполнено неравенство

$$
\left.\left|\int_{B_{R}}\right| \nabla u_{j}\right|^{p(x)-2} \nabla u_{j} \cdot \nabla \psi d x \mid<\varepsilon .
$$

Поэтому (см. доказательство теоремы 3.1) последовательность $\left\{u_{j}\right\}$ фундаментальна в $H_{0}\left(B_{R}\right)$, а предельная функция $u \in H_{0}\left(B_{R}\right)$ удовлетворяет равенству (5.10). В силу строгой выпуклости функционала решение единственно. Поскольку минимизирующая последовательность состоит из функций множества (5.2), то по предложению $2.3 u=1$ на $K$ в смысле $H\left(B_{R}\right)$. Теорема доказана.

ОПРЕДЕЛЕНИЕ 5.1. Функция, на которой реализуется нижняя грань функционала (1.17), называется емкостным потенциалом $K$ (относительно шара $B_{R}$ ).

Для того чтобы привести свойства емкостного потенциала, нам потребуется еше одно понятие.

ОПРЕДЕЛЕНИЕ 5.2. Функция $u \in H(D)$ назьвается суперрешением уравнения (1.1) в области $D$, если

$$
\int_{D}|\nabla u|^{p(x)-2} \nabla u \cdot \nabla \psi d x \geqslant 0
$$

для всех неотрицательных почти всюду в $D$ функций $\psi \in H_{0}(D)$. 
Tеорема 5.2. Емкостный потенииал $и(x)$ компакта $K \subset B_{R}$ является $H$-решением уравнения (1.1) в области $B_{R} \backslash K$ и имеет граничные значения $u=1$ на $\partial K u и=0$ на $\partial B_{R}$ в смысле $H\left(B_{R}\right)$. Более того, $u(x)$ суперрешение уравнения (1.1) в $B_{R}$.

ДоКАЗАТЕЛЬСТВО. Поскольку емкостный потенциал $u(x)$ является минимизантом задачи (1.17), то $(F(u+t \nabla \varphi)-F(u)) t^{-1} \geqslant 0$ для любого $t>0$ и произвольной неотрицательной на $K$ функции $\varphi \in C_{0}^{\infty}\left(B_{R}\right)$. Повторяя рассуждения теоремы 3.2 , придем к неравенству

$$
\int_{B_{R}}|\nabla u|^{p(x)-2} \nabla u \cdot \nabla \varphi d x \geqslant 0 .
$$

Пусть $\psi \in H_{0}(D)$ и $\psi(x) \geqslant 0$ почти всюду в $B_{R}$. Поскольку $\psi(x)=\psi^{+}(x)=$ $-\left(-\psi^{-}(x)\right)$, то из определения класса $H_{0}(D)$ и свойств а) и б) леммы 2.5 следует существование последовательности неотрицательных в $B_{R}$ функций $\psi_{j} \in$ $C_{0}^{\infty}\left(B_{R}\right)$, сходящейся к $\psi(x)$ в $W_{0}\left(B_{R}\right)$. Полагая в неравенстве $(5.12) \varphi=\psi_{j}$, после перехода к пределу придем к соотношению (5.11), из которого следует, что функция $u(x)$ является суперрешением уравнения (1.1) в смысле данного выше определения. Далее, так как любую функцию $\varphi \in C_{0}^{\infty}\left(B_{R}\right)$ с компактным носителем в $B_{R} \backslash K$ можно подставить в $(5.12)$, мы выводим, что $u(x)$ является решением уравнения (1.1) в $B_{R} \backslash K$. Принятие граничных значений $u=1$ на $\partial K$ и $u=0$ на $\partial B_{R}$, понимаемых в смысле $H\left(B_{R}\right)$, вытекает из того, что $u \in H_{0}\left(B_{R}\right)$ и $u=1$ на $K$ в смысле $H\left(B_{R}\right)$. Теорема доказана.

Емкостный потенциал $u(x)$ компакта $K \subset B_{R}$ удовлетворяет неравенству (5.12) для любой неотрицательной на $K$ функции $\psi \in C_{0}^{\infty}\left(B_{R}\right)$. По теореме Щварца [37] сушествует неотрицательная мера $\mu$ на $K$ такая, что

$$
\int_{B_{R}}|\nabla u|^{p(x)-2} \nabla u \cdot \nabla \psi d x=\int_{B_{R}} \psi d \mu \quad \forall \psi \in C_{0}^{\infty}\left(B_{R}\right) .
$$

Поскольку $u=1$ на $K$ в смысле $H\left(B_{R}\right)$, то носитель меры $\mu$ находится на $\partial K$. Найденную меру по аналогии со случаем, когда $p=$ const, назовем емкостнылм распределением $K$.

Из (5.13) вытекает, что емкостное распределение удовлетворяет условию (1.10). Рассмотрим равенство (5.13) на последовательности $\left\{\psi_{j}\right\}$, сходящейся в $W_{0}\left(B_{R}\right)$ к емкостному потенциалу и состоящей из функций $\psi_{j} \in C_{0}^{\infty}\left(B_{R}\right)$, равных единице в окрестности $K$. Поскольку носитель меры находится на $K$, то выражение в правой части равно $\mu(K)$ для любого $j$, в то время как левая часть стремится к интегралу, эквивалентному емкости:

$$
p_{1} C_{p}\left(K, B_{R}\right) \leqslant \int_{B_{R}}|\nabla u|^{p(x)} d x \leqslant p_{2} C_{p}\left(K, B_{R}\right),
$$

где $p_{1}, p_{2}-$ постоянные из условия (1.2). Таким образом, мы доказали, что

$$
p_{1} C_{p}\left(K, B_{R}\right) \leqslant \mu(K) \leqslant p_{2} C_{p}\left(K, B_{R}\right) .
$$

Прежде чем переходить к доказательству следуюшего утверждения, отметим, что если мера $\mu$ является емкостным распределением компакта $K \subset B_{R}$, то ее сужение на борелевское множество $E \subset B_{R}$ в $B_{R}$ удовлетворяет условию (1.10), которое в силу теоремы 3.3 влечет однозначную разрешимость задачи (1.9) в шаре $B_{R}$. 
ЛЕмма 5.1. Если $\mu$-емкостное распределение компакта $K \subset B_{R}$, то для любого компакта $E \subset B_{R}$ выполнено неравенство

$$
\mu(E) \leqslant C(p) C_{p}\left(K \cap E, B_{R}\right) .
$$

ДокАЗАТЕЛЬСтво. Пусть мера $\nu$ является сужением емкостного распределения $\mu$ на компакт $F$. Обозначим через $u_{\mu}$ емкостньй потенциал $K$, а через $u_{\nu}$ peшение задачи Дирихле

$$
L u_{\nu}=-d \nu \text { в } B_{R}, \quad u_{\nu} \in H_{0}\left(B_{R}\right),
$$

однозначная разрешимость которой вытекает из сделанного выше замечания о выполнении свойства (1.10) для меры $\nu$. Сначала покажем, что $u_{\nu}(x)$ удовлетворяет неравенству

$$
0 \leqslant u_{\nu}(x) \leqslant u_{\mu}(x) \text { почти всюду в } B_{R} .
$$

Покажем оценку сверху. Так как функция $v(x)=u_{\mu}(x)-u_{\nu}(x)$ принадлежит классу $H_{0}\left(B_{R}\right)$, то по свойствам а) и б) леммы 2.5 сушествует последовательность неотрицательных в $B_{R}$ функций $v_{j} \in C_{0}^{\infty}\left(B_{R}\right)$, сходящаяся в $W_{0}\left(B_{R}\right)$ к $v^{-}(x)$. Выбирая в интегральных тождествах для $u_{\nu}(x)$ и $u_{\mu}(x)$ пробную функцию $v_{j}(x)$, получим

$$
\int_{B_{R}}\left(\left|\nabla u_{\mu}\right|^{p(x)-2} \nabla u_{\mu}-\left|\nabla u_{\nu}\right|^{p(x)-2} \nabla u_{\nu}\right) \cdot \nabla v_{j} d x \leqslant 0 .
$$

После перехода к пределу придем к неравенству

$$
\int_{G}\left(\left|\nabla u_{\mu}\right|^{p(x)-2} \nabla u_{\mu}-\left|\nabla u_{\nu}\right|^{p(x)-2} \nabla u_{\nu}\right) \cdot \nabla\left(u_{\mu}-u_{\nu}\right) d x \leqslant 0,
$$

где $G=\left\{x: u_{\mu}(x)<u_{\nu}(x)\right\}$, из которого вытекает требуемая оценка сверху. Аналогично выводится и оценка снизу.

Переходя к доказательству (5.16), возьмем в интегральном тождестве для $u_{\nu}(x)$ пробную функцию $\psi \in C_{0}^{\infty}\left(B_{R}\right)$, равную единице в окрестности компакта $E \subset B_{R}$. После применения неравенства Юнга получим

$$
\begin{aligned}
\mu(E) & =\int_{B_{R}} \psi d \mu=\int_{B_{R}}\left|\nabla u_{\nu}\right|^{p(x)-2} \nabla u_{\nu} \cdot \nabla \psi d x \\
& \leqslant \int_{B_{R}}\left|\nabla u_{\nu}\right|^{p(x)} d x+C(p) \int_{B_{R}} \frac{|\nabla \psi|^{p(x)}}{p(x)} d x .
\end{aligned}
$$

Оценим сверху первый интеграл в правой части (5.18). Рассмотрим функцию $\varphi(x)=\max \left(\psi(x), u_{\nu}(x)\right)$, принадлежащую, как нетрудно видеть, классу $H_{0}\left(B_{R}\right)$. Ясно, что $\psi-\varphi \in H_{0}\left(B_{R} \backslash E\right), \varphi-u_{\nu} \in H_{0}\left(B_{R}\right)$ и $\varphi-u_{\nu} \geqslant 0$ почти всюду в $B_{R}$. Поскольку $u_{\nu}(x)$ - суперрешение в $B_{R}$ и решение в $B_{R} \backslash E$, то

$$
\begin{gathered}
\int_{B_{R}}\left|\nabla u_{\nu}\right|^{p(x)-2} \nabla u_{\nu} \cdot \nabla\left(\psi-u_{\nu}\right) d x=\int_{B_{R}}\left|\nabla u_{\nu}\right|^{p(x)-2} \nabla u_{\nu} \cdot \nabla\left(\varphi-u_{\nu}\right) d x \\
+\int_{B_{R} \backslash E}\left|\nabla u_{\nu}\right|^{p(x)-2} \nabla u_{\nu} \cdot \nabla(\psi-\varphi) d x \geqslant 0 .
\end{gathered}
$$


Отсюда получим, что

$$
\int_{B_{R}}\left|\nabla u_{\nu}\right|^{p(x)} d x \leqslant \int_{B_{R}}\left|\nabla u_{\nu}\right|^{p(x)-1}|\nabla \psi| d x,
$$

и по неравенству Юнга имеем

$$
\int_{B_{R}}\left|\nabla u_{\nu}\right|^{p(x)} d x \leqslant C(p) \int_{B_{R}} \frac{|\nabla \psi|^{p(x)}}{p(x)} d x .
$$

Поэтому в силу (5.18)

$$
\mu(E) \leqslant C(p) \int_{B_{R}} \frac{|\nabla \psi|^{p(x)}}{p(x)} d x .
$$

Минимизируя интеграл, стоящий в правой части этого неравенства, придем к требуемому неравенству (5.16). Лемма доказана.

\section{§6. Неравенство Харнака слабого типа}

1. Интегральные оценки решений. Всюду далее предполагается, что показатель суммируемости $p(x)$, удовлетворяющий в области $D$ условиям $(1.2)$ и (1.12), продолжен на все $\mathbb{R}^{n}$ с сохранением своих свойств. Рассмотрим неотрицательное решение задачи Дирихле

$$
L u=0 \quad \text { в } D, \quad u \in W(D), \quad(u-f) \in W_{0}(D)
$$

с граничной функцией $f \in C^{\infty}(\bar{D}), f(x) \geqslant 0$ при $x \in \bar{D}$. Нашей целью будет доказательство интегральных оценок $u(x)$ в шарах $B_{R}$ достаточно малого радиуса $R<1 / 16$ с центром в граничной точке $x_{0} \in \partial D$. Ниже

$$
\begin{gathered}
s=\sup _{B_{4 R}} p, \quad \bar{s}=\inf _{B_{4 R}} p, \quad p_{0}=p\left(x_{0}\right), \\
m=\inf _{\partial D \cap B_{4 R}} f, \quad M=\max _{\partial D} f, \\
u_{m}(x)=\left\{\begin{array}{ll}
\min (u(x), m), & x \in D \cap B_{4 R}, \\
m, & x \in B_{4 R} \backslash D,
\end{array} \quad v_{m}(x)=u_{m}(x)+R,\right.
\end{gathered}
$$

a $\eta(x)$ - функция из $C_{0}^{\infty}\left(B_{4 R}\right)$, удовлетворяющая неравенствам $0 \leqslant \eta(x) \leqslant 1$. Отметим, что по принципу максимума точная верхняя грань решения $u(x)$ не превосходит постоянной $M$.

Пользуясь обозначением (4.1), будем применять теорему вложения Соболева в виде

$$
\begin{gathered}
\left(f_{B_{R}}|\varphi|^{k q} d x\right)^{1 / k} \leqslant C R^{q} f_{B_{R}}|\nabla \varphi|^{q} d x, \\
q \geqslant 1, \quad k=\frac{n}{n-1}, \quad \varphi \in C_{0}^{\infty}\left(B_{R}\right) .
\end{gathered}
$$

Предварительно приведем одно вспомогательное утверждение, в котором $\sigma=$ $\inf _{B_{r}} p(x)$, a $B_{r} \subset B_{4 R}$ - произвольный шар радиуса $r$ с центром, совпадающим с центром $B_{4 R}$. 
ПРЕДЛОЖЕНИЕ 6.1. Для любых $r \leqslant 4 R u \gamma \leqslant \gamma_{0}<0$ справедливо неравенство

$$
\int_{B_{r}} v_{m}^{\gamma-1}\left|\nabla v_{m}\right|^{\sigma} \eta^{s} d x \leqslant C\left(p, \gamma_{0}\right) \int_{B_{4 R}}\left(v_{m}^{\gamma-1}+v_{m}^{\gamma+p(x)-1}|\nabla \eta|^{p(x)}\right) d x .
$$

ДоКАЗАТЕЛЬСТво. Выбирая в интегральном тождестве (1.4) пробную функцию

$$
\psi(x)=\left[v_{m}^{\gamma}(x)-(m+R)^{\gamma}\right] \eta^{s}(x),
$$

которая, как нетрудно видеть, принадлежит классу $W_{0}(D)$, придем к оценке

$$
\left|\gamma_{0}\right| \int_{B_{4 R}} v_{m}^{\gamma-1}\left|\nabla v_{m}\right|^{p(x)} \eta^{s} d x \leqslant s \int_{B_{4 R}} v_{m}^{\gamma}\left|\nabla v_{m}\right|^{p(x)-1}|\nabla \eta| \eta^{s-1} d x .
$$

Отсюда, по неравенству Юнга, примененному к подьнтегральному выражению в правой части, имеем

$$
\int_{B_{r}} v_{m}^{\gamma-1}\left|\nabla v_{m}\right|^{p(x)} \eta^{s} d x \leqslant C\left(p, \gamma_{0}\right) \int_{B_{4 R}} v_{m}^{\gamma+p(x)-1}|\nabla \eta|^{p(x)} d x
$$

и неравенство (6.6) следует из оценки $v_{m}^{\gamma-1}\left|\nabla v_{m}\right|^{\sigma} \eta^{s} \leqslant v_{m}^{\gamma-1}\left|\nabla v_{m}\right|^{p(x)} \eta^{s}+v_{m}^{\gamma-1} \eta^{s}$, вьполненной почти всюду в $B_{r}$. Предложение доказано.

Введем для неотрицательных функций $w(x)$ и постоянных $q \neq 0$ функционал

$$
\Phi\left(q, B_{r}, w\right)=\left(f_{B_{r}} w^{q} d x\right)^{1 / q}
$$

Лемма 6.1. Существуют положительные постоянные $q_{0}$ и С, зависящие только от $n, p$ и $M$, такие, что

$$
\Phi\left(q_{0}, B_{3 R}, v_{m}\right) \leqslant C \Phi\left(-q_{0}, B_{3 R}, v_{m}\right) .
$$

ДокАЗАТЕЛЬСТво. Пусть $B_{2 r} \subset B_{4 R}$ и $\eta \in C_{0}^{\infty}\left(B_{2 r}\right)$ - срезающая функция: $\eta=1$ в $B_{r},|\nabla \eta| \leqslant 2 / r$. Выбирая в (6.6) $\gamma=1-\sigma$, будем иметь

$$
\int_{B_{r}}\left|\nabla \ln v_{m}\right|^{\sigma} d x \leqslant C(n, p) \int_{B_{2 r}}\left(v_{m}^{-\sigma}+v_{m}^{p(x)-\sigma} r^{-p(x)}\right) d x .
$$

Поскольку $v_{m} \geqslant R$ в $B_{4 R}$, то $v_{m}^{-\sigma} \leqslant r^{-\sigma}$, а в силу логарифмического условия (1.12) имеем $v_{m}^{p-\sigma} \leqslant C(p, M)$ и $r^{-p(x)} \leqslant C(p) r^{-\sigma}$ при $x \in B_{2 r}$. С учетом перечисленных соотношений и неравенства Гёльдера получим

$$
\int_{B_{r}}\left|\nabla \ln v_{m}\right| d x \leqslant C(n, p, M) r^{n-1} .
$$

Для завершения доказательства осталось воспользоваться леммой Джона-Ниренберга [38]. Лемма доказана. 
Лемма 6.2. Для любого $q<0$ справедливо неравенство

$$
\Phi\left(q, B_{3 R}, v_{m}\right) \leqslant C(n, p, q, M) \inf _{B_{R}} v_{m}
$$

ДокАЗАТЕЛьСтво. Полагая в неравенстве (6.6) $B_{r}=B_{2 R}, \sigma=\bar{s}$ и $\gamma=\beta-$ $\bar{s}+1$, где $\beta<0$, получим

$$
\int_{B_{4 R}} v_{m}^{\beta-\bar{s}}\left|\nabla v_{m}\right|^{\bar{s}} \eta^{s} d x \leqslant C(p) \int_{B_{4 R}}\left(v_{m}^{\beta-\bar{s}}+v_{m}^{\beta-\bar{s}+p(x)}|\nabla \eta|^{p(x)}\right) d x .
$$

Перепишем последнюю оценку в виде

$$
\begin{aligned}
\int_{B_{4 R}}\left|\nabla\left(v_{m}^{\beta / \bar{s}} \eta\right)\right|^{\bar{s}} d x \leqslant & C(p)(1+|\beta|)^{s}\left(\int_{B_{4 R}} v_{m}^{\beta}|\nabla \eta|^{\bar{s}} d x\right. \\
& \left.+\int_{B_{4 R}}\left(v_{m}^{\beta-\bar{s}}+v_{m}^{\beta-\bar{s}+p(x)}|\nabla \eta|^{p(x)}\right) d x\right) .
\end{aligned}
$$

Пусть $R \leqslant \rho<r \leqslant 3 R$, а срезаюшая функция $\eta$ такова, что $\eta=1$ в $B_{\rho}$ и $|\nabla \eta| \leqslant C r(R(r-\rho))^{-1}$. Поскольку $v_{m}^{p-\bar{s}} \leqslant C(p, M), v_{m}^{-\bar{s}} \leqslant R^{-\bar{s}}$ и согласно условию (1.12)

$$
|\nabla \eta|^{p} \leqslant C(p) R^{-\bar{s}}\left(\frac{r}{r-\rho}\right)^{s}, \quad x \in B_{4 R},
$$

то, учитывая эти соотношения, оценим интеграл в левой части (6.11) по неравенству теоремы вложения (6.5). В результате придем к оценке

$$
\left(f_{B_{\rho}} v_{m}^{k \beta} d x\right)^{1 / k} \leqslant C(n, p, M)(1+|\beta|)^{s}\left(\frac{r}{r-\rho}\right)^{s} f_{B_{r}} v_{m}^{\beta} d x
$$

из которой следует, что

$$
\Phi\left(\beta, B_{r}, v_{m}\right) \leqslant C^{1 /|\beta|}(1+|\beta|)^{s /|\beta|}\left(\frac{r}{r-\rho}\right)^{s /|\beta|} \Phi\left(k \beta, B_{\rho}, v_{m}\right),
$$

где $C=C(n, p, M)$. Проитерируем это соотношение. Для $j=0,1, \ldots$ обозначим $r_{j}=R+2^{-j+1} R, \chi_{j}=q k^{j}$ и выберем в (6.12) $r=r_{j}, \rho=r_{j+1}, \beta=-\chi_{j}$. Полагая $\Psi_{j}=\Phi\left(-\chi_{j}, B_{r_{j}}, v_{m}\right)$, имеем

$$
\Psi_{j} \leqslant C^{1 / \chi_{j}}\left[\left(1+\chi_{j}\right) 2^{j}\right]^{s / \chi_{j}} \Psi_{j+1}
$$

Теперь по индукции $\Psi_{0} \leqslant C(n, p, q, M) \lim _{j \rightarrow \infty} \Psi_{j}$, и поскольку $\Psi_{j} \rightarrow \inf _{B_{R}} v_{m}$ при $j \rightarrow \infty$, приходим к требуемому неравенству (6.10). Лемма доказана.

ЛЕмма 6.3. Для любых $0<q_{1}<q_{2}<n\left(p_{0}-1\right) /(n-1)$ выполнено неравенство

$$
\Phi\left(q_{2}, B_{5 R / 2}, v_{m}\right) \leqslant C\left(n, p, q_{1}, q_{2}, M\right) \Phi\left(q_{1}, B_{3 R}, v_{m}\right)
$$


ДокаЗАТЕЛьСтво. Возьмем для $5 R / 2 \leqslant \rho<r \leqslant 3 R$ срезающую функцию $\eta(x)$ леммы 6.2 и воспользуемся неравенством (6.6), полагая в нем $B_{r}=B_{4 R}, \sigma=\bar{s}$ и $\gamma=1-p_{0}+\beta$, где $0<\beta \leqslant q_{2} / k, k=n /(n-1)$. Так как $v_{m}^{-p_{0}} \geqslant v_{m}^{-\bar{s}}$ ив силу (1.12) $v_{m}^{-p_{0}} \leqslant C(p) v_{m}^{-\bar{s}}$, то имеющееся соотношение можно переписать в виде

$$
\int_{B_{4 R}} v_{m}^{\beta-\bar{s}}\left|\nabla v_{m}\right|^{\bar{s}} \eta^{s} d x \leqslant C(p) \int_{B_{4 R}}\left(v_{m}^{\beta-\bar{s}}+v_{m}^{\beta-\bar{s}+p(x)}|\nabla \eta|^{p(x)}\right) d x .
$$

Повторяя рассуждения леммы 6.2, как и при вьводе (6.12), придем к неравенству

$$
\Phi\left(k \beta, B_{\rho}, v_{m}\right) \leqslant C(n, p, \beta, M)\left(\frac{r}{r-\rho}\right)^{s / \beta} \Phi\left(\beta, B_{r}, v_{m}\right)
$$

Для $j=0,1, \ldots$ положим $r_{j}=3 R-2^{-j-1} R, \chi_{j}=q_{2} k^{-j}$. Пусть $\chi_{j_{0}+1} \leqslant q_{1}<\chi_{j_{0}}$ и $\Psi_{j}=\Phi\left(\chi_{j}, B_{r_{j}}, v_{m}\right)$. Выбирая в (6.14) $r=r_{j+1}, \rho=r_{j}$ и $\beta=\chi_{j+1}$, получим

$$
\Psi_{j} \leqslant C\left(n, p, q_{2}, j\right) \Psi_{j+1}
$$

Итерируя данное соотношение для номеров $j=0,1, \ldots, j_{0}$, будем иметь

$$
\Psi_{0}=\Phi\left(q_{2}, B_{5 R / 2}, v_{m}\right) \leqslant C\left(n, p, q_{1}, q_{2}, M\right) \Phi\left(\tilde{q}, B_{3 R}, v_{m}\right)
$$

где $\tilde{q}=\chi_{j_{0}+1} \leqslant q_{1}$. Если теперь $\tilde{q}=q_{1}$, то сразу приходим к $(6.13)$, а если $\tilde{q}<q_{1}$, то (6.13) вытекает из неравенства Гёльдера. Лемма доказана.

Теорема 6.1 (неравенство Харнака слабого типа). Для любого $0<q<$ $n\left(p_{0}-1\right) /(n-1)$ имеет место неравенство

$$
\left(\int_{B_{5 R / 2}} v_{m}^{q} d x\right)^{1 / q} \leqslant C(n, p, q, M) \inf _{B_{R}} v_{m}
$$

ДокАЗАТЕльство. Пусть $q_{0}$ - постоянная леммы 6.1. Выбирая в неравенстве (6.10) $q=-q_{0}$ и пользуясь $(6.9)$, получим

$$
\Phi\left(q_{0}, B_{3 R}, v_{m}\right) \leqslant C(n, p, q, M) \inf _{B_{R}} v_{m}
$$

Если $q \leqslant q_{0}$, то $(6.15)$ следует из $(6.16)$ и неравенства Гёльдера, а в случае $q>q_{0}$ нужно дополнительно воспользоваться оценкой (6.13) с постоянными $q_{1}=q_{0}$, $q_{2}=q$. Теорема доказана.

2. Неравенство Харнака слабого типа для суперрешений. Неравенство Харнака слабого типа имеет место и для неотрицательных ограниченных суперрешений уравнения (1.1) в области $D$.

ЛЕмма 6.4. Если $и(x)$ - неотрицательное, ограниченное суперрешение уравнения (1.1) в области $D$, то в произвольном шаре $B_{R}=B_{R}^{x_{0}}$ таком, что 
$B_{4 R} \subset D$, для любого $0<q<n\left(p_{0}-1\right) /(n-1)$, где $p_{0}=p\left(x_{0}\right)$, справедливо неравенство

$$
\left(f_{B_{2 R}} u^{q} d x\right)^{1 / q} \leqslant C(n, p, q, M)\left(\inf _{B_{R}} u+R\right),
$$

в котором $M=\sup _{D} u(x)$.

ДоКАЗАТЕЛЬСТВО. Положим $v(x)=u(x)+R$ и заметим, что для любых $r \leqslant$ $4 R$ и $\gamma \leqslant \gamma_{0}<0$ выполнено неравенство

$$
\int_{B_{r}} v^{\gamma-1}|\nabla v|^{\sigma} \eta^{s} d x \leqslant C\left(p, \gamma_{0}\right) \int_{B_{4 R}}\left(v^{\gamma-1}+v^{\gamma+p(x)-1}|\nabla \eta|^{p(x)}\right) d x
$$

где $\eta \in C_{0}^{\infty}\left(B_{4 R}\right), 0 \leqslant \eta \leqslant 1$. Для вывода $(6.18)$ необходимо в интегральном неравенстве (5.11) выбрать пробную функцию $\psi(x)=v^{\gamma}(x) \eta^{s}(x)$ и повторить рассуждения, использованные при доказательстве оценки (6.6). Теперь таким же способом, каким оценка (6.15) была получена из (6.6), установим оценку

$$
\left(f_{B_{5 R / 2}} v^{q} d x\right)^{1 / q} \leqslant C(n, p, q, M) \inf _{B_{R}} v
$$

влекушую (6.17). Лемма доказана.

Важным следствием неравенства (6.17) является возможность представления ограниченного суперрешения полунепрерывной снизу функцией.

ЛЕмма 6.5. Если $u \in W(D)$ является ограниченным суперрешением уравнения (1.1), то существует полунепрерывная снизу функщия $\hat{u}(x)$ такая, что $u(x)=\hat{u}(x)$ почти всюду в $D$ и для любой точки $x \in D$

$$
\hat{u}(x)=\sup \lim _{y \rightarrow x} v(y),
$$

где точная верхняя грань берется по всем функциям $v$, совпадающим $c \hat{u}$ почти всюду в $D$.

ДокАЗАТЕЛЬСТво. Переходя, если нужно, к функции $\sup _{D} u+u(x)$, можно считать, что рассматриваемое суперрешение неотрицательно в $D$. Покажем в произвольной точке $x \in D$ равенство

$$
\sup \underline{\lim }_{y \rightarrow x} u(y)=\lim _{r \rightarrow 0} f_{B_{r}^{x}} u(x) d x .
$$

Для этого зафиксируем $x \in D$ и рассмотрим шар $B_{r}^{x}$ достаточно малого радиуса, удовлетворяющий условию $B_{4 r}^{x} \subset D$. Пусть $M=\sup _{D} u$ и $m_{r}=\inf _{B_{r}^{x}} u$. Из неравенства Харнака слабого типа (6.17) для $0<q<1$ имеем

$$
\begin{aligned}
0 & \leqslant f_{B_{r}^{x}}\left(u-m_{r}\right) d x \leqslant\left(M-m_{r}\right)^{(q-1) / q}\left(f_{B_{r}^{x}}\left(u-m_{r}\right)^{q} d x\right)^{1 / q} \\
& \leqslant C(n, p, q, M)\left(r+m_{r / 2}-m_{r}\right),
\end{aligned}
$$

откуда и вытекает (6.20). По теореме Лебега правая часть в (6.20) совпадает с $u(x)$ почти всюду в $D$. Поэтому после изменения $u(x)$ на множестве меры нуль придем к функции $\hat{u}(x)$, удовлетворяющей равенству (6.19). Полунепрерывность снизу $\hat{u}(x)$ является следствием (6.19). Лемма доказана. 
3. Оценка типа Мозера. Далее $B_{R}$ - шар с центром в замыкании области $D$. Нашей целью является доказательство мозеровской оценки для ограниченного и неотрицательного в $D \cap B_{4 R}$ решения $u(x)$ уравнения (1.1). Если $B_{4 R} \cap \partial D \neq \varnothing$, то предполагается, что решение имеет нулевой след на $B_{4 R} \cap D$, или, более точно, принадлежит классу $W_{0}\left(D \cap B_{4 R}, \partial D \cap B_{4 R}\right)$. В этом случае будем считать $u(x)=0$ при $x \in B_{4 R} \backslash D$.

В следующей лемме используются обозначения из (6.2) и полагается $v(x)=$ $u(x)+R, M=\sup _{D \cap B_{4 R}} u$.

ЛЕмма 6.6. Справедливо неравенство

$$
\sup _{D \cap B_{R}} u \leqslant C(n, p, M)\left(\int_{B_{2 R}} u d x+R\right) .
$$

ДокАЗАТЕльСтво. Выбирая в интегральном тождестве (1.4) пробную функцию

$$
\psi(x)=\left(v^{\beta}(x)-R^{\beta}\right) \eta^{s}(x), \quad \beta \geqslant 1, \quad \eta \in C_{0}^{\infty}\left(B_{4 R}\right), \quad 0 \leqslant \eta(x) \leqslant 1,
$$

получим

$$
\beta \int_{B_{4 R}}|\nabla v|^{p(x)} v^{\beta-1} \eta^{s} d x \leqslant s \int_{B_{4 R}}|\nabla v|^{p(x)-1} v^{\beta}|\nabla \eta| d x .
$$

После применения неравенства Юнга с соответствуюшим $\varepsilon$ найдем

$$
\int_{B_{4 R}}|\nabla v|^{p(x)} v^{\beta-1} \eta^{s} d x \leqslant C(p) \int_{B_{4 R}} v^{\beta+p(x)-1}|\nabla \eta|^{p(x)} d x .
$$

Далее, $v^{\beta+p(x)-1} \leqslant C(p, M) v^{\beta+\bar{s}-1}$, и, пользуясь в $B_{4 R}$ неравенством

$$
|\nabla v|^{\bar{s}} v^{\beta-1} \eta^{s} \leqslant|\nabla v|^{p(x)} v^{\beta-1} \eta^{s}+v^{\beta-1} \eta^{s}
$$

получим

$$
\int_{B_{4 R}}|\nabla v|^{\bar{s}} v^{\beta-1} \eta^{s} d x \leqslant C(p, M)\left(\int_{B_{4 R}} v^{\beta-1} \eta^{s} d x+\int_{B_{4 R}} v^{\beta+\bar{s}-1}|\nabla \eta|^{p}(x) d x\right) .
$$

Применяя теорему вложения Соболева (6.5), придем к оценке

$$
\begin{aligned}
\left(\int_{B_{4 R}}\right. & \left.\left(v^{\beta+\bar{s}-1} \eta^{s}\right)^{k} d x\right)^{1 / k} \leqslant C(n, p, M)(\beta+\bar{s}-1)^{s} R^{\bar{s}} \\
& \times\left(f_{B_{4 R}} v^{\beta-1} \eta^{s} d x+f_{B_{4 R}} v^{\beta+\bar{s}-1}|\nabla \eta|^{p(x)} d x\right) .
\end{aligned}
$$

Пусть $R \leqslant \rho<r \leqslant 3 R, \eta \in C_{0}^{\infty}\left(B_{r}\right), \eta=1$ в $B_{\rho}$ и $|\nabla \eta| \leqslant C r(R(r-\rho))^{-1}$. Пользуясь условием (1.12) и неравенством $v^{\beta-1} \leqslant R^{-\bar{s}} v^{\beta+\bar{s}-1}$, перепишем (6.22) в виде

$$
\left(\int_{B_{\rho}} v^{k(\beta+\bar{s}-1)} d x\right)^{1 / k} \leqslant C(n, p, M)(\beta+\bar{s}-1)^{s}\left(\frac{r}{r-\rho}\right)^{s} f_{B_{r}} v^{\beta+\bar{s}-1} d x .
$$


Проитерируем это неравенство. Для $j=0,1, \ldots$ обозначим $r_{j}=\rho+2^{-j}(r-\rho)$, $\chi_{j}=k^{j} \bar{s}$ и выберем в (6.23) $r=r_{j}, \rho=r_{j+1}, \beta=\chi_{j}-\bar{s}+1$. Полагая

$$
\Phi_{j}=\Phi\left(\chi_{j}, B_{r_{j}}, v\right)
$$

где выражение в правой части определено в (6.8), получим соотношение

$$
\Phi_{j+1} \leqslant C^{1 / \chi_{j}}\left(2^{j} \chi_{j}\right)^{s / \chi_{j}}\left(\frac{r}{r-\rho}\right)^{s / \chi_{j}} \Phi_{j}
$$

в котором $C=C(n, p, M)$. Итерируя это неравенство, найдем, что

$$
\sup _{B_{\rho}} v \leqslant C(n, p, M)\left(\frac{r}{r-\rho}\right)^{a} \Phi\left(\bar{s}, B_{r}, v\right), \quad a=a(p)>0 .
$$

Отсюда требуемая оценка (6.21) вытекает в силу рассуждений, приведенных в доказательстве леммы 3.2 работы [39]. Лемма доказана.

\section{§7. Достаточное условие регулярности граничной точки}

1. Вспомогательные интегральные оценки. Приведем интегральные оценки для неотрицательного решения задачи (6.1) с граничной функцией $f \in$ $C^{\infty}(\bar{D}), f(x) \geqslant 0$ при $x \in \bar{D}$. Предполагается, что показатель суммируемости $p(x)$ продолжен в $\mathbb{R}^{n}$ с сохранением условий (1.2) и (1.12). Ниже используются обозначения (6.2)-(6.4), а $B_{R}^{x_{0}}-$ шар с центром в граничной точке $x_{0} \in \partial D$ достаточно малого радиуса $R<1 / 16$. Следуюшие вспомогательные утверждения являются следствиями теоремы 6.1.

ЛЕмма 7.1. Если $0<q<p_{0}-1$, mo

$$
f_{B_{2 R}^{x_{0}}} v_{m}^{q-p_{0}}\left|\nabla v_{m}\right|^{p(x)} d x \leqslant C(n, p, q, M) R^{-p_{0}}\left(\inf _{B_{R}^{x_{0}}} v_{m}\right)^{q} .
$$

ДоказАТЕльСТво. Возьмем срезающую функцию $\eta \in C_{0}^{\infty}\left(B_{5 R / 2}^{x_{0}}\right), \quad \eta=1$ в $B_{2 R},|\nabla \eta| \leqslant C R^{-1}$, и выберем в неравенстве (6.7) $\gamma=q+1-p_{0}, r=2 R$. Пользуясь условием (1.12) и ограниченностью $u(x)$, в силу чего $R^{-p(x)} \leqslant C(p) R^{-p_{0}}$ и $v_{m}^{p(x)-p_{0}} \leqslant C(M, p)$, получим

$$
f_{B_{2 R}^{x_{0}}} v_{m}^{q-p_{0}}\left|\nabla v_{m}\right|^{p} d x \leqslant C(n, p, q, M) R^{-p_{0}} \int_{B_{5 R / 2}^{x_{0}}} v_{m}^{q} d x
$$

Теперь неравенство (7.1) вытекает из (6.15). Лемма доказана.

ЛЕмМа 7.2. Справедливо неравенство

$$
f_{B_{2 R}^{x_{0}}}\left|\nabla v_{m}\right|^{p(x)-1} d x \leqslant C(n, p, M) R^{1-p_{0}}\left(\inf _{B_{R}^{x_{0}}} v_{m}\right)^{p_{0}-1} .
$$


ДоКАЗАТЕЛЬСТВО. Пусть $\tau=\min \left(p_{0}, n /(n-1)\right), t=(1+\tau) / 2$ и постоянная $\lambda=\lambda(x)$, где $x \in B_{2 R}^{x_{0}}$, такова, что

$$
\lambda^{-p(x)}=R^{1-p_{0}}\left(f_{B_{2 R}^{x_{0}}} v_{m}^{t\left(p_{0}-1\right)} d x\right)^{(1-t) / t} .
$$

По неравенству Юнга имеем

$$
\begin{aligned}
f_{B_{2 R}^{x_{0}}} & \left|\nabla v_{m}\right|^{p(x)-1} d x \leqslant f_{B_{2 R}^{x_{0}}} v_{m}^{-t}\left|\nabla v_{m}\right|^{p(x)} \lambda^{p(x) /(p(x)-1)} d x \\
& +R^{1-p_{0}}\left(f_{B_{2 R}^{x_{0}}} v_{m}^{t\left(p_{0}-1\right)} d x\right)^{(1-t) / t} \int_{B_{2 R}^{x_{0}}} v_{m}^{t(p(x)-1)} d x .
\end{aligned}
$$

Пользуясь условием (1.12) и неравенством $R \leqslant v_{m}(x) \leqslant M+R$, выполненным в $B_{4 R}^{x_{0}}$, получим

$$
\begin{gathered}
\lambda^{p(x) /(p(x)-1)} \leqslant C(n, p, M) R\left(\int_{B_{2 R}^{x_{0}}} v_{m}^{t\left(p_{0}-1\right)} d x\right)^{(t-1) / t\left(p_{0}-1\right)} \\
\int_{B_{2 R}^{x_{0}}} v_{m}^{t(p(x)-1)} d x \leqslant C(n, p, M) f_{B_{2 R}^{x_{0}}} v_{m}^{t\left(p_{0}-1\right)} d x
\end{gathered}
$$

Учитывая в (7.3) оценки (7.4) и (7.5), имеем

$$
\begin{aligned}
f_{B_{2 R}^{x_{0}}}\left|\nabla v_{m}\right|^{p(x)-1} d x \leqslant & C(n, p, M) R\left(f_{B_{2 R}^{x_{0}}} v_{m}^{-t}\left|\nabla v_{m}\right|^{p(x)} d x\right) \\
& \times\left(f_{B_{2 R}^{x_{0}}} v_{m}^{t\left(p_{0}-1\right)} d x\right)^{\frac{t-1}{t\left(p_{0}-1\right)}} \\
& +C(n, p, M) R^{1-p_{0}}\left(f_{B_{2 R}^{x_{0}}} v_{m}^{t\left(p_{0}-1\right)} d x\right)^{1 / t} .
\end{aligned}
$$

Для доказательства (7.2) осталось, используя выбор $t$, оценить первый интеграл в (7.6) по неравенству (7.1), а остальные - по неравенству (6.15). Лемма доказана.

В следующей лемме полагается

$$
\gamma(R)=C_{p}\left(\bar{B}_{R}^{x_{0}} \backslash D, B_{2 R}^{x_{0}}\right) R^{p_{0}-n},
$$

а функция $u_{m}(x)$ имеет тот же смысл, что и в (6.4).

ЛЕмма 7.3. Справедливо неравенство

$$
(R+m)(\gamma(R))^{1 /\left(p_{0}-1\right)} \leqslant C_{0}(n, p, M)\left(\inf _{B_{R}^{x_{0}}} u_{m}+R\right) .
$$


ДокАЗАТЕЛЬСТво. Пусть $\eta \in C_{0}^{\infty}\left(B_{2 R}^{x_{0}}\right), \quad \eta=1$ в $B_{R}^{x_{0}}$ и $|\nabla \eta| \leqslant C R^{-1}$. Выбирая в интегральном тождестве (1.4) пробную функцию $\psi(x)=(m+R-$ $\left.v_{m}(x)\right) \eta^{s}(x)$, получим

$$
\begin{aligned}
\int_{B_{2 R}^{x_{0}}}\left|\nabla v_{m}\right|^{p(x)} \eta^{s} d x & \leqslant C(p) R^{-1} \int_{B_{2 R}^{x_{0}}}\left|\nabla v_{m}\right|^{p(x)-1}\left(m+R-v_{m}\right) d x \\
& \leqslant C(p) R^{-1}(m+R) \int_{B_{2 R}^{x_{0}}}\left|\nabla v_{m}\right|^{p(x)-1} d x .
\end{aligned}
$$

Полагая $w_{m}(x)=v_{m}(x) /(m+R)$, имеем

$$
\begin{aligned}
& \int_{B_{2 R}^{x_{0}}}(m+R)^{p(x)}\left|\nabla\left(w_{m} \eta\right)\right|^{p(x)} d x \\
& \quad \leqslant C(p)(m+R)\left(R^{-1} \int_{B_{2 R}^{x_{0}}}\left|\nabla v_{m}\right|^{p(x)-1} d x+\int_{B_{2 R}^{x_{0}}} v_{m}^{p(x)-1} R^{-p(x)} d x\right) .
\end{aligned}
$$

Согласно логарифмическому условию (1.12) и ограниченности $u(x)$ в шаре $B_{2 R}^{x_{0}}$ выполнены неравенства

$$
(m+R)^{p(x)} \geqslant C(p)(m+R)^{p_{0}}, \quad R^{-p(x)} \leqslant C(p) R^{-p_{0}}, \quad v_{m}^{p(x)-1} \leqslant C(p, M) v_{m}^{p_{0}-1} .
$$

Применяя еще оценки (6.15) и (7.2) к интегралам в правой части (7.8), найдем, что

$$
(m+R)^{p_{0}-1} \int_{B_{2 R}^{x_{0}}}\left|\nabla\left(w_{m} \eta\right)\right|^{p(x)} d x \leqslant C(n, p, M) R^{n-p_{0}}\left(\inf _{B_{R}^{x_{0}}} u_{m}+R\right)^{p_{0}-1} .
$$

Поскольку $w_{m}(x) \eta(x)=1$ при $x \in \bar{B}_{R}^{x_{0}} \backslash D$ и $w_{m} \eta \in W_{0}\left(B_{2 R}^{x_{0}}\right)$, то, пользуясь определением емкости, придем к требуемому соотношению (7.7). Лемма доказана.

В случае $p_{0}>n$ нам потребуется следующее утверждение.

ЛЕмма 7.4. Если функщия $u \in W(D)$, удовлетворяющая условию $|u(x)| \leqslant 1$ в $D$, является решением задачи (6.1) с граничной функиией $f \in C^{\infty}(\bar{D})$, равной нулю для $x \in \partial D \cap B_{\rho}^{x_{0}}$, то при $\rho<\rho_{0}(n, p)$ u $r \leqslant \rho / 4$ имеет место неравенство

$$
\sup _{D \cap B_{r}^{x_{0}}}|u(x)| \leqslant C(n, p)\left(\frac{r}{\rho}\right)^{1-n / p_{0}} .
$$

ДоКАЗАТЕЛЬСТВо. Поскольку $p_{0}>n$, то $p(x)>n$ в $\bar{B}_{\rho}^{x_{0}}$ для достаточно малых $\rho<\rho_{0}(p, n)$. Положим

$$
l=\sup _{B_{\rho}^{x_{0}}} p(x), \quad \sigma=\inf _{B_{r}^{x_{0}}} p(x), \quad D_{t}^{x_{0}}=D \cap B_{t}^{x_{0}}, \quad M(t)=\sup _{D_{t}^{x_{0}}}|u(x)| .
$$


По теореме вложения Соболева, неравенству Юнга и логарифмическому условию (1.12) имеем

$$
\begin{aligned}
M(r) & \leqslant C(n, p) r^{1-n / \sigma}\left(\int_{D_{r}^{x_{0}}}|\nabla u|^{\sigma} d x\right)^{1 / \sigma} \\
& \leqslant C(n, p)\left(r^{1-n / p_{0}}\left(\int_{D_{\rho / 4}^{x_{0}}}|\nabla u|^{p(x)} d x\right)^{1 / \sigma}+r\right) .
\end{aligned}
$$

Для оценки интеграла в правой части (7.10) возьмем срезающую функцию $\eta \in$ $C_{0}^{\infty}\left(B_{\rho}^{x_{0}}\right), \eta=1$ в $B_{\rho / 4}^{x_{0}},|\nabla \eta| \leqslant C \rho^{-1}$, и выберем в интегральном тождестве (1.4) пробную функцию $\psi(x)=u(x) \eta^{l}(x)$. После несложного подсчета, используюшего условие (1.12) и неравенство $|u(x)| \leqslant 1$, получим оценку

$$
\left(\int_{D_{\rho / 4}^{x_{0}}}|\nabla u|^{p(x)} d x\right)^{1 / \sigma} \leqslant C(p)\left(\int_{D_{\rho}^{x_{0}}}|u|^{p(x)} \rho^{-p(x)} d x\right)^{1 / \sigma} \leqslant C(p) \rho^{-1+n / p_{0}}
$$

Отсюда в силу (7.10) имеем

$$
M(r) \leqslant C(n, p)\left(\left(\frac{r}{\rho}\right)^{1-n / p_{0}}+r\right)
$$

что влечет (7.9). Лемма доказана.

2. Доказательство теоремы 1.2. Докажем при $p_{0} \leqslant n$ оценку модуля непрерывности (1.20) в предположении расходимости в нуле интеграла (1.19). Сначала рассмотрим решение $u(x)$ задачи (6.1) с граничной функцией $f \in C^{\infty}(\bar{D})$. Пусть

$$
M=\max _{\partial D}|f|, \quad F_{R}=\sup _{\partial D \cap B_{R}} f, \quad f_{R}=\inf _{\partial D \cap B_{R}} f, \quad M_{R}=\sup _{D \cap B_{R}} u, \quad m_{R}=\inf _{D \cap B_{R}} u .
$$

Применяя неравенство (7.7) к функциям $M_{4 R}-u(x)$ и $u(x)-m_{4 R}$, получим

$$
\begin{aligned}
\left(M_{4 R}-F_{4 R}+R\right) \mu(R) & \leqslant C_{0}\left(M_{4 R}-M_{R}+R\right), \\
\left(f_{R}-m_{4 R}+R\right) \mu(R) & \leqslant C_{0}\left(m_{R}-m_{4 R}+R\right),
\end{aligned}
$$

где $\mu(R)=(\gamma(R))^{1 /\left(p_{0}-1\right)}$. Суммируя эти оценки, придем к соотношению

$$
\underset{D \cap B_{R}}{\operatorname{OSC}} u \leqslant(1-\sigma \mu(R)) \underset{D \cap B_{4 R}}{\operatorname{OSC}} u+\sigma \mu(R) \underset{\partial D \cap B_{4 R}}{\operatorname{OSC}} f+R
$$

с постоянной $\sigma=1 / C_{0}$. Проитерируем это неравенство. Для $j=0,1, \ldots$ и $0<$ $\rho<1 / 16$ положим $r_{j}=4^{-j} \rho, \omega_{j}=\operatorname{osc}_{D \cap B_{r_{j}}} u, f_{j}=\operatorname{osc}_{\partial D \cap B_{r_{j}}} f, \mu_{j}=\mu\left(r_{j}\right)$ и перепишем неравенство (7.11) в виде

$$
\omega_{j} \leqslant\left(1-\sigma \mu_{j}\right) \omega_{j-1}+\sigma \mu_{j} f_{j-1}+r_{j}
$$


Отсюда по индукции в предположении $\prod_{i=0}^{k} a_{i}=1$ при $k<0$ для $j \geqslant 1$ будем иметь

$$
\begin{aligned}
\omega_{j} \leqslant & \omega_{0} \prod_{i=0}^{j-1}\left(1-\sigma \mu_{j-i}\right)+\sum_{k=0}^{j-1} \mu_{j-k} f_{j-k-1} \prod_{i=0}^{k-1}\left(1-\sigma \mu_{j-i}\right) \\
& +\sum_{k=0}^{j-1} r_{j-k} \prod_{i=0}^{k-1}\left(1-\sigma \mu_{j-i}\right) .
\end{aligned}
$$

Для оценки полученных произведений заметим, что

$$
\prod_{i=0}^{k}\left(1-\sigma \mu_{j-i}\right) \leqslant \exp \left(-\theta_{0} \sum_{i=0}^{k} \mu_{j-i}\right),
$$

где $\theta_{0}=$ const $\cdot \sigma$. Поскольку

$$
\begin{gathered}
C_{1}\left(n, p_{0}\right) \int_{r_{j-i+1}}^{r_{j-i}} \frac{\mu(\tau)}{\tau} d \tau \leqslant \mu_{j-i} \leqslant C_{2}\left(n, p_{0}\right) \int_{r_{j-i}}^{r_{j-i-1}} \frac{\mu(\tau)}{\tau} d \tau, \\
\sum_{k=0}^{j-1} r_{j-k} \prod_{i=0}^{k-1}\left(1-\sigma \mu_{j-i}\right) \leqslant \rho
\end{gathered}
$$

то из (7.12) придем к оценке

$$
\begin{aligned}
\omega_{j} \leqslant & \omega_{0} \exp \left(-\theta \int_{r_{j+1}}^{\rho} \frac{\mu(\tau)}{\tau} d \tau\right) \\
& +C\left(n, p_{0}\right) f_{0} \sum_{k=0}^{j-1} \exp \left(-\theta \int_{r_{j+1}}^{r_{j+1-k}} \frac{\mu(\tau)}{\tau} d \tau\right) \int_{r_{j-k}}^{r_{j-k-1}} \frac{\mu(\tau)}{\tau} d \tau+\rho
\end{aligned}
$$

Пользуясь неравенством $\mu(\tau) \leqslant C(n, p)$, получим

$$
\begin{aligned}
& \sum_{k=0}^{j-1} \exp \left(-\theta \int_{r_{j+1}}^{r_{j+1-k}} \frac{\mu(\tau)}{\tau} d \tau\right) \int_{r_{j-k}}^{r_{j-k-1}} \frac{\mu(\tau)}{\tau} d \tau \\
& \quad \leqslant \sum_{k=0}^{j-1} C(n, p, M) \int_{r_{j-k}}^{r_{j-k-1}} \frac{\mu(t)}{t} \exp \left(-\theta \int_{r_{j+1}}^{t} \frac{\mu(\tau)}{\tau} d \tau\right) d t \\
& \leqslant C(n, p, M) \int_{r_{j}}^{\rho} \frac{\mu(t)}{t} \exp \left(-\theta \int_{r_{j}}^{t} \frac{\mu(\tau)}{\tau} d \tau\right) d t \leqslant C(n, p, M)
\end{aligned}
$$

и из (7.13) после соотвествуюшего выбора $j$ при $r \leqslant \rho / 4$ будем иметь

$$
\underset{D \cap B_{r}^{x_{0}}}{\operatorname{osc}} u \leqslant C(n, p, M)\left(\underset{\partial D \cap B_{\rho}^{x_{0}}}{\operatorname{Oos}} f+\rho+\underset{\partial D}{\operatorname{osc}} f \exp \left(-\theta \int_{r}^{\rho} \frac{\mu(\tau)}{\tau} d \tau\right)\right) .
$$

При выполнении условия (1.19) отсюда следует сушествование предела решения $u(x)$ при $x \rightarrow x_{0}, x \in D$. Покажем, что этот предел совпадает со значением 
$f\left(x_{0}\right)$ граничной функции в точке $x_{0}$. Предположим противное. Тогда в силу непрерывности $f(x)$ в $\bar{D}$ следует существование положительных постоянных $\varepsilon_{0}$ и $r$ таких, что $|u(x)-f(x)| \geqslant \varepsilon_{0}$ для всех $x \in D \cap B_{r}^{x_{0}}$. Не ограничивая обшности, будем считать $u(x)-f(x) \geqslant \varepsilon_{0}$ при $x \in D \cap B_{r}^{x_{0}}$. Положим $E_{r}=\bar{B}_{r}^{x_{0}} \backslash D$ и покажем, что $C_{p}\left(E_{r / 2}, B_{r}^{x_{0}}\right)=0$. Пусть $\widetilde{E}_{r}=\partial D \cap \bar{B}_{r}^{x_{0}}$ и $w(x)=(u(x)-f(x)) / \varepsilon_{0}$. Так как $w \in W_{0}(D)$, то $w \in W_{0}\left(D \cap B_{r}^{x_{0}}, \widetilde{E}_{r}\right)$ и усеченная функция $v(x)=\min (w(x), 1)$ равна нулю на $\widetilde{E}_{r}$ в смысле $W\left(D \cap B_{r}^{x_{0}}\right)$. С другой стороны, в силу сделанного предположения $w \geqslant 1$ на $\widetilde{E}_{r}$ в смысле $W\left(D \cap B_{r}^{x_{0}}\right)$ и $v(x)=1$ на $\widetilde{E}_{r}$ в смысле $W\left(D \cap B_{r}^{x_{0}}\right)$. Поэтому функция, тождественно равная единице в $D \cap B_{r}^{x_{0}}$, принадлежит классу $W_{0}\left(D \cap B_{r}^{x_{0}}, \widetilde{E}_{r}\right)$. Отсюда вытекает принадлежность классу $W_{0}\left(B_{r}^{x_{0}}, E_{r}\right)$ функции $\eta(x)$, равной единище в $D \cap B_{r}^{x_{0}}$ и нулю на $E_{r}$. Ясно, что $|\nabla \eta|=0$ почти всюду в $B_{r}^{x_{0}}$, и из теоремы вложения (см. [30])

$$
\int_{B_{r}^{x_{0}}}(1-\eta)^{2} d x \leqslant C\left(n, D \cap B_{r}^{x_{0}}\right) \int_{B_{r}^{x_{0}}}|\nabla(1-\eta)|^{2} d x
$$

получаем равенство нулю лебеговой меры $\left|E_{r}\right|$ множества $E_{r}$ :

$$
\left|E_{r}\right|=\int_{B_{r}^{x_{0}}}(1-\eta)^{2} d x \leqslant C \int_{B_{r}^{x_{0}}}|\nabla(1-\eta)|^{2} d x=0 .
$$

Рассмотрим теперь функцию $\psi(x)=\varphi(x)(1-\eta(x))$, где $\varphi \in C_{0}^{\infty}\left(B_{r}^{x_{0}}\right)$ и $\varphi(x)=1$ в окрестности $E_{r / 2}$. Поскольку $\psi \in W_{0}\left(B_{r}^{x_{0}}\right)$ и $\psi=1$ на $E_{r / 2}$ в смысле $W\left(B_{r}^{x_{0}}\right)$, то

$$
C_{p}\left(E_{r / 2}, B_{r}^{x_{0}}\right) \leqslant \int_{B_{r}^{x_{0}}}|\nabla \psi|^{p(x)} d x=\int_{E_{r}}|\nabla \varphi|^{p(x)} d x=0 .
$$

Таким образом, $C_{p}\left(E_{r / 2}, B_{r}^{x_{0}}\right)=0$, что противоречит расходимости интеграла в (1.19). Следовательно, предел решения $u(x)$ при $x \rightarrow x_{0}, x \in D$, совпадает с $f\left(x_{0}\right)$, и оценку $(7.14)$ для $r \leqslant \rho / 4$ можно записать в виде

$$
\begin{aligned}
\left|u(x)-f\left(x_{0}\right)\right| \leqslant & \left.C(n, p, M)\left(\underset{\underset{D \cap B_{\rho}^{x}}{\operatorname{osc}_{0}} f+\rho}{\rho} f+\frac{\mu(\tau)}{\tau} d \tau\right)\right) \forall x \in D \cap B_{r}^{x_{0}} . \\
& +\underset{\partial D}{\operatorname{osc}} f \exp \left(-\theta \int_{r}^{\rho} \frac{\frac{(x)}{\tau}}{}\right.
\end{aligned}
$$

Применяя (7.15) к решениям $u_{k}(x)$ задач (1.16), участвуюших в определении обобщенного решения $u_{f}(x)$ задачи $(1.15)$ с непрерьвной граничной функцией $f(x)$, будем иметь

$$
\begin{aligned}
\left|u_{k}(x)-f_{k}\left(x_{0}\right)\right| \leqslant & C\left(n, p, M_{k}\right)\left(\underset{D \cap B_{\rho}^{x_{0}}}{\operatorname{osc}} f_{k}+\rho\right. \\
& \left.+\underset{\partial D}{\operatorname{osc}} f_{k} \exp \left(-\theta \int_{r}^{\rho} \frac{\mu(\tau)}{\tau} d \tau\right)\right) \forall x \in D \cap B_{r}^{x_{0}}
\end{aligned}
$$


где $M_{k}$ - максимум $\left|f_{k}(x)\right|$ на $\partial D$. Поскольку последовательность $\left\{u_{k}\right\}$ сходится к $u_{f}(x)$ равномерно на компактных подмножествах $D$ и последовательность $\left\{f_{k}\right\}$ сходится к $f(x)$ равномерно на $\bar{D}$, то, пользуясь равномерной ограниченностью постоянных $C\left(n, p, M_{k}\right)$ из $(7.16)$, после перехода к пределу придем к требуемой оценке (1.20).

Предположим теперь, что $p_{0}>n$, и сначала рассмотрим решение задачи (6.1) с граничной функцией $f \in C^{\infty}(\bar{D})$. По теореме вложения Соболева это решение непрерывно в $B_{\rho}^{x_{0}} \cap \bar{D}$ для достаточно малых $\rho$.

Пусть $0<\alpha<1 / 2, \beta=1+\alpha, \sigma=\inf _{B_{2 \rho}^{x_{0}}} p(x), v(x)$ - емкостный потенциал точки $x_{0}$ относительно шара $B_{\beta \rho}^{x_{0}}$, а радиус $\rho$ столь мал, что $p(x)>n$ в $B_{2 \rho}^{x_{0}}$.

Емкостный потенциал непрерывен в $B_{\beta \rho}^{x_{0}}$, и нам понадобится его оценка при $x \in$ $D \cap S_{\rho}^{x_{0}}$. По теореме вложения Соболева, неравенству Юнга и условию (1.12) имеем

$$
\begin{aligned}
v(x) & \leqslant C(n, p)(\alpha \rho)^{1-n / \sigma}\left(\int_{B_{\alpha \rho}^{x}}|\nabla v|^{\sigma} d x\right)^{1 / \sigma} \\
& \leqslant C(n, p) \alpha^{1-n / \sigma}\left(\rho^{1-n / p_{0}}\left(\int_{B_{\beta \rho}^{x_{0}}}|\nabla v|^{p(x)} d x\right)^{1 / \sigma}+\rho\right) .
\end{aligned}
$$

В силу (5.14) и (5.8) получим

$$
\int_{B_{\beta \rho}^{x_{0}}}|\nabla v|^{p(x)} d x \leqslant C(n, p)
$$

и из (7.17) следует, что

$$
v(x) \leqslant \frac{1}{2}, \quad x \in D \cap B_{\rho}^{x_{0}}, \quad \alpha \leqslant \alpha_{0}(n, p) .
$$

Перейдем к выводу оценки (1.21). Согласно (7.18) при соответствующем выборе $\alpha$ будем иметь

$$
u(x)-\inf _{D \cap B_{\rho}^{x_{0}}} u \leqslant 2 \operatorname{osc}_{D \cap B_{\rho}^{x_{0}}} u(1-v(x)), \quad x \in D \cap S_{\rho}^{x_{0}} .
$$

С другой стороны,

$$
u(x)-\inf _{D \cap B_{\rho}^{x_{0}}} u \leqslant \operatorname{osc}_{\partial D \cap B_{\rho}^{x_{0}}} f, \quad x \in \partial D \cap \bar{B}_{\rho}^{x_{0}} .
$$

Следовательно, при $x \in \partial\left(D \cap B_{\rho}^{x_{0}}\right)$

$$
u(x)-\inf _{D \cap B_{\rho}^{x_{0}}} u \leqslant \operatorname{osc}_{\partial D \cap B_{\rho}^{x_{0}}} f+2 \underset{D \cap B_{\rho}^{x_{0}}}{\operatorname{osc}} u(1-v(x)) .
$$

По принщипу максимума данное неравенство имеет место и при $x \in D \cap B_{\rho}^{x_{0}}$. В силу оценки $(7.9)$, примененной к функции $1-v(x)$, имеем

$$
u(x)-\inf _{D \cap B_{\rho}^{x_{0}}} u \leqslant \operatorname{osc}_{\partial D \cap B_{\rho}^{x_{0}}} f+C(n, p) \underset{\partial D}{\operatorname{osc}} f\left(\frac{r}{\rho}\right)^{1-n / p_{0}}, \quad x \in D \cap B_{r}^{x_{0}}, \quad r \leqslant \frac{\rho}{4} .
$$


Отсюда вытекает неравенство

$$
\underset{D \cap B_{r}^{x_{0}}}{\operatorname{Osc}} u \leqslant \underset{\partial D \cap B_{\rho}^{x_{0}}}{\operatorname{Osc}} f+C(n, p) \underset{\partial D}{\operatorname{Osc}} f\left(\frac{r}{\rho}\right)^{1-n / p_{0}}
$$

исходя из которого доказательство (1.21) завершается так же, как и в случае $p_{0} \leqslant n$. Теорема доказана.

Из теоремы 1.2 следует, что расходимость в нуле интеграла (1.19) является достаточным условием регулярности граничной точки $x_{0} \in \partial D$.

3. Доказательство теоремы 1.3. Если внешность области $D$ в окрестности граничной точки $x_{0}$ содержит конус с вершиной в $x_{0}$, то для достаточно малых $t$ существует шар $B_{\delta t}^{x^{\prime}}$ радиуса $\delta t$ с постоянной $\delta$, зависящей от раствора конуса, такой, что $B_{\delta t}^{x^{\prime}} \subset E_{t}$. В силу свойств емкости, нижней оценки (5.9) и условия (1.12) имеем

$$
C_{p}\left(E_{t}, B_{2 t}^{x_{0}}\right) \geqslant C_{p}\left(\bar{B}_{\delta t}^{x^{\prime}}, B_{2 t}^{x_{0}}\right) \geqslant C_{p}\left(\bar{B}_{\delta t}^{x^{\prime}}, B_{2 t}^{x^{\prime}}\right) \geqslant C t^{n-p\left(x^{\prime}\right)} \geqslant C t^{n-p_{0}}
$$

Поэтому начиная с достаточно малых $\rho$ получим

$$
\begin{gathered}
\int_{r}^{\rho}(\gamma(t))^{1 /\left(p_{0}-1\right)} t^{-1} d t \geqslant C \ln \left(\frac{r}{\rho}\right) \\
\exp \left(-\theta \int_{r}^{\rho}(\gamma(t))^{1 /\left(p_{0}-1\right)} t^{-1} d t\right) \leqslant\left(\frac{r}{\rho}\right)^{\beta_{0}} .
\end{gathered}
$$

Отсюда и из неравенства (1.20) для гёльдеровой в точке $x_{0}$ граничной функции $f(x)$ с показателем Гёльдера $\beta$ имеем

$$
\sup _{B_{r}^{x_{0}} \cap D}\left|u_{f}(x)-f\left(x_{0}\right)\right| \leqslant C\left(\rho^{\beta}+\left(\frac{r}{\rho}\right)^{\beta_{0}}\right), \quad r \leqslant \frac{\rho}{4}
$$

Выбирая здесь $\rho=r^{1 / 2}$, будем иметь

$$
\sup _{B_{r}^{x_{0}} \cap D}\left|u_{f}(x)-f\left(x_{0}\right)\right| \leqslant C\left(r^{\beta / 2}+r^{\beta_{0} / 2}\right) \leqslant C r^{\alpha},
$$

где $\alpha=\min \left(\beta / 2, \beta_{0} / 2\right)$. Теорема доказана.

\section{§8. Необходимое условие регулярности граничной точки}

1. Вспомогательные результаты. Пусть $D \subset \mathbb{R}^{n}$ - ограниченная область, в которой изучается обобшенное решение задачи $(1.15), x_{0} \in \partial D$ - граничная точка. Продолжим показатель суммируемости $p(x)$ в $\mathbb{R}^{n} \backslash D$ с сохранением условий (1.2) и (1.12). Вопрос о регулярности граничной точки $x_{0} \in \partial D$ задачи Дирихле (1.15) тесно связан с поведением в окрестности $x_{0}$ емкостного потенциала 
$u(x)=u_{\rho}(x)$ компакта $E_{\rho}=\left(\mathbb{R}^{n} \backslash D\right) \cap \bar{B}_{\rho}^{x_{0}}$ относительно шара $B_{8 \rho}^{x_{0}}$. Напомним, что функция $u(x)$ принадлежит классу $H_{0}\left(B_{8 \rho}^{x_{0}}\right)$ и удовлетворяет в $B_{8 \rho}^{x_{0}}$ уравнению

$$
\operatorname{div}\left(|\nabla u|^{p(x)-2} \nabla u\right)=-d \mu
$$

с мерой $\mu=\mu_{\rho}$, реализующей емкость $E_{\rho}$. Решение понимается в смысле интегрального тождества

$$
\int_{B_{8 \rho}^{x_{0}}}|\nabla u|^{p(x)-2} \nabla u \cdot \nabla \psi d x=\int_{B_{8 \rho}^{x_{0}}} \psi d \mu
$$

на пробных функциях $\psi \in C_{0}^{\infty}\left(B_{R}\right)$. Поскольку емкостный потенциал является неотрицательным в $B_{8 \rho}^{x_{0}}$ суперрешением, то, основываясь на утверждении леммы 6.5 , будем рассматривать его как полунепрерывную снизу функцию в $B_{8 \rho}^{x_{0}}$. Всюду далее предполагается, что $p_{0}=p\left(x_{0}\right) \leqslant n$.

ЛЕмма 8.1. Если существует постоянная $\rho>0$, для которой емкостной потенииал $u(x)=u_{\rho}(x)$ удовлетворяет неравенству

$$
u\left(x_{0}\right)=\lim _{x \rightarrow x_{0}} u(x)<1,
$$

то граничная точка $x_{0}$ иррегулярна.

ДоКАЗАТЕЛЬСТво. Сначала приведем используемое ниже следствие предположения (8.2). В силу полунепрерывности снизу $u(x)$ имеем

$$
u\left(x_{0}\right)=\varliminf_{x \rightarrow x_{0}} u(x)=\sup \underline{\lim }_{x \rightarrow x_{0}} \tilde{u}(x)<1,
$$

где точная верхняя грань берется по всем функциям $\tilde{u}(x)$, совпадающим с $u(x)$ почти всюду. Емкостный потенциал $u(x)$ равен единице почти всюду на $E_{\rho}$. Более того, функция $u(x)$ непрерывна в области $B_{8 \rho}^{x_{0}} \backslash E_{\rho}$, где совпадает с решением $w(x)$ задачи Дирихле

$$
L w=0 \quad \text { в } \quad B_{8 \rho}^{x_{0}} \backslash E_{\rho},\left.\quad w\right|_{\partial B_{8 \rho}^{x_{0}}}=0,\left.\quad w\right|_{\partial E_{\rho}}=1 .
$$

Поэтому согласно (8.3) имеем

$$
u\left(x_{0}\right)=\lim _{\substack{x \rightarrow x_{0} \\ x \in B_{8 \rho}^{x} \backslash E_{\rho}}} u(x)<1 .
$$

Отметим, что если данное соотношение выполнено для емкостного потенциала $u(x)=u_{\rho}(x)$, то по принципу максимума оно будет иметь место и для емкостного потенщиала $u_{\rho^{\prime}}(x)$ при $\rho^{\prime}<\rho$.

Обозначим через $u_{f}(x)$ решение задачи Дирихле (1.15) с гладкой граничной функцией $f(x)$, удовлетворяюшей условиям: $f(x)=3 / 2$ при $x \in \partial D \cap B_{r / 2}^{x_{0}}$, 
$0 \leqslant f(x)<3 / 2$ при $x \in \partial D \cap\left(B_{r}^{x_{0}} \backslash B_{r / 2}^{x_{0}}\right)$ и $f(x)=0$ на оставшейся части границы $\partial D$. Докажем для $\rho<\rho_{0}(n, p)$ и $r<r_{0}(n, p, \rho)$ неравенство

$$
u_{f}(x) \leqslant \frac{1}{2}+u(x), \quad x \in D \cap B_{8 \rho}^{x_{0}} .
$$

Будем считать, что $D \cap \partial B_{8 \rho}^{x_{0}} \neq \varnothing$. Поскольку $u_{f}(x) \leqslant \frac{1}{2}+u(x)$ на множестве $\partial D \cap \bar{B}_{8 \rho}^{x_{0}}$ в смысле $W_{0}\left(D \cap B_{8 \rho}^{x_{0}}\right)$ (см. $\left.\S 3\right)$ и $u(x)=0$ на $\partial B_{8 \rho}^{x_{0}}$ в том же смысле, то по принципу максимума достаточно установить поточечную оценку

$$
u_{f}(x)<\frac{1}{2}, \quad x \in D \cap \partial B_{8 \rho}^{x_{0}} .
$$

Пусть $B_{\rho}$ - произвольный шар с центром на $\partial B_{8 \rho}^{x_{0}} \cap D$. Если $\partial D \cap B_{2 \rho} \neq \varnothing$, то $u_{f} \in W_{0}\left(D \cap B_{2 \rho}, \partial D \cap B_{2 \rho}\right)$, и, полагая $u_{f}(x)=0$ при $x \in B_{2 \rho} \backslash D$, по неравенству (6.21) будем иметь

$$
\sup _{B_{\rho / 2}} u_{f}(x) \leqslant C(n, p)\left(f_{B_{\rho}} u_{f}(x) d x+\rho\right) .
$$

Для оценки интеграла в правой части (8.7) рассмотрим вспомогательную функцию $v_{r}(x)$, являюшуюся решением задачи Дирихле

$$
L v_{r}=0 \quad \text { в } \quad B_{R}^{x_{0}} \backslash E_{r},\left.\quad v_{r}\right|_{\partial B_{R}^{x_{0}}}=0,\left.\quad v_{r}\right|_{\partial E_{r}}=\frac{3}{2},
$$

где $R$ - диаметр области $D$. Положим $v_{r}(x)=3 / 2$ при $x \in E_{r}$. По принципу максимума $u_{f}(x) \leqslant v_{r}(x)$ при $x \in D$, и с учетом (8.7) имеем

$$
\sup _{B_{\rho / 2}} u_{f}(x) \leqslant C(n, p)\left(\rho^{-n} \int_{B_{R}^{x_{0}}} v_{r} d x+\rho\right) .
$$

Заметим, что функция $v_{r}(x)$ реализует точную нижнюю грань функционала $F(\varphi)$ (см. (5.1)) по множеству функций $\varphi \in C_{0}^{\infty}\left(B_{R}^{x_{0}}\right)$, равных $3 / 2$ в окрестности $E_{r}$. Отсюда и из определения емкости компакта $E_{r}$ вытекает неравенство

$$
\int_{B_{R}^{x_{0}}}\left|\nabla v_{r}\right|^{p(x)} d x \leqslant \operatorname{const}(p) C_{p}\left(E_{r}, B_{R}^{x_{0}}\right) \leqslant \operatorname{const}(p) C_{p}\left(\bar{B}_{r}^{x_{0}}, B_{8 \rho}^{x_{0}}\right),
$$

а из верхних оценок емкости шара (5.4), взятых для значений $p_{0} \leqslant n$, следует, что $v_{r} \rightarrow 0$ в $W_{0}\left(B_{R}^{x_{0}}\right)$ при $r \rightarrow 0$. В частности, $v_{r} \rightarrow 0$ в $L_{1}\left(B_{R}^{x_{0}}\right)$. Поэтому в силу (8.8) при $\rho<\rho_{0}(n, p)$ и $r<r_{0}(n, p, \rho)$ справедливы и неравенство (8.6), и требуемое соотношение (8.5). Таким образом согласно (8.5) и (8.4) имеем

$$
\lim _{\substack{x \rightarrow x_{0} \\ x \in D}} u_{f}<\frac{3}{2},
$$

что влечет иррегулярность граничной точки $x_{0}$. Лемма доказана.

Доказательство следующих двух утверждений, в которых получены оценки введенного вьше емкостного потенциала $u(x)=u_{\rho}(x)$, основано на схеме рассуждений работы [27], где $p(x) \equiv$ const. Напомним, что $p_{0} \leqslant n$, и для $r \leqslant 2 \rho$ положим

$$
\bar{s}=\inf _{B_{r}^{x_{0}}} p(x), \quad s=\sup _{B_{r}^{x_{0}}} p(x), \quad G_{r}^{a}=\left\{x: x \in B_{r}^{x_{0}}, u(x)>a\right\} .
$$


ЛЕмма 8.2. Если $r \leqslant 4 \rho$ и существуют постоянные $d \geqslant r$ u $\gamma \in\left(p_{0}-1\right.$, $\left.n\left(p_{0}-1\right) /\left(n-p_{0}+1\right)\right)$ такие, что для $a \in[0,1)$ выполнено соотношение

$$
\left|G_{2 r}^{a}\right| \leqslant 2^{-1} d^{-\gamma} \int_{G_{r}^{a}}(u-a)^{\gamma} d x
$$

то при $\rho \leqslant \rho_{0}(n, p, \gamma)$ функиия $и(x)$ удовлетворяет неравенству

$$
\begin{aligned}
d^{-\gamma} r^{-n} & \int_{G_{r}^{a}}(u-a)^{\gamma} d x \leqslant C(n, \gamma, p) \\
& \times\left(d^{-\gamma} r^{-n} \int_{G_{2 r}^{a}}(u-a)^{\gamma} d x+d^{1-p_{0}} r^{p_{0}-n} \mu\left(B_{2 r}^{x_{0}}\right)\right)^{q / p_{0}}
\end{aligned}
$$

в котором $q=q\left(p_{0}, \gamma\right)>p_{0}$.

ДоКАЗАТЕЛЬСТВО. Не ограничивая обшности, будем считать $a=0$. В этом случае

$$
\left|G_{2 r}^{0}\right| \leqslant 2^{-1} d^{-\gamma} \int_{G_{r}^{0}} u^{\gamma} d x
$$

и нетрудно заметить, что согласно предположению (8.9)

$$
\int_{G_{2 r}^{0}}\left(1+\frac{u}{d}\right)^{\gamma} d x \leqslant C(\gamma) d^{-\gamma} \int_{G_{2 r}^{0}} u^{\gamma} d x
$$

В силу (8.11) имеем

$$
\begin{gathered}
d^{-\gamma} \int_{G_{r}^{0} \backslash G_{r}^{d}} u^{\gamma} d x \leqslant 2^{-1} d^{-\gamma} \int_{G_{r}^{0}} u^{\gamma} d x \\
d^{-\gamma} \int_{G_{r}^{0}} u^{\gamma} d x \leqslant 2 d^{-\gamma} \int_{G_{r}^{d}} u^{\gamma} d x .
\end{gathered}
$$

Поскольку $0 \leqslant u(x) \leqslant 1$, то отсюда и из $(8.9)$ вытекает неравенство $d \leqslant 1$. Кроме того, $d \geqslant r$ по условию леммы, так что

$$
r \leqslant d \leqslant 1
$$

Для оценки интеграла в правой части (8.13) положим

$$
q=\frac{p_{0} \gamma}{p_{0}-\gamma /\left(p_{0}-1\right)}
$$

и рассмотрим вспомогательную функцию

$$
w(x)=\left(1+\frac{u(x)}{d}\right)^{\gamma / q}-1
$$


Отметим, что

$$
q>p_{0}, \quad q<\frac{n \bar{s}}{n-\bar{s}}, \quad \rho \leqslant \rho_{0}(n, p, \gamma)
$$

Сначала проверим неравенство

$$
d^{-\gamma} \int_{G_{r}^{d}} u^{\gamma} d x \leqslant C(\gamma) \int_{G_{r}^{d}} w^{q} d x
$$

Оно заведомо выполнено с постоянной $C=C_{0}^{-q}$, если только

$$
\left(1+\frac{u(x)}{d}\right)^{\gamma / q}-1 \geqslant C_{0}\left(\frac{u(x)}{d}\right)^{\gamma / q}, \quad x \in G_{r}^{d}
$$

Поскольку

$$
\inf _{u \geqslant d}\left(\left(1+\frac{u}{d}\right)^{\gamma / q}-1\right)\left(1+\frac{u}{d}\right)^{-\gamma / q}=1-2^{-\gamma / q},
$$

то в качестве $C_{0}$ можно взять $1-2^{-\gamma / q}$. Таким образом, в силу $(8.13)$ и $(8.17)$ имеем

$$
d^{-\gamma} r^{-n} \int_{G_{r}^{0}} u^{\gamma} d x \leqslant C(\gamma) \int_{B_{r}^{x_{0}}} w^{q} d x
$$

Оценим интеграл в правой части этого неравенства. Возьмем срезающую функцию $\eta \in C_{0}^{\infty}\left(B_{2 r}^{x_{0}}\right), 0 \leqslant \eta \leqslant 1, \eta=1$ в $B_{r}^{x_{0}},|\nabla \eta| \leqslant 2 / r$. Так как $q>\bar{s}$ и при $\bar{s}<n$ константа $q$ не превосходит критического показателя Соболева (см. (8.16)), то из теоремы вложения, примененной к функции $w \eta^{s / \bar{s}}$, следует, что

$$
\int_{B_{r}^{x_{0}}} w^{q} d x \leqslant C(n, \bar{s})\left(r^{\bar{s}} f_{B_{2 r}^{x_{0}}}|\nabla w|^{\bar{s}} \eta^{s} d x+\int_{B_{2 r}^{x_{0}}} w^{\bar{s}} d x\right)^{q / \bar{s}}
$$

По неравенству Гёльдера, соотношению (8.12) и предположению (8.11) имеем

$$
\begin{aligned}
\int_{B_{2 r}^{x_{0}}} w^{\bar{s}} d x & \leqslant\left(\int_{G_{2 r}^{0}}\left(1+\frac{u}{d}\right)^{\gamma} d x\right)^{\bar{s} / q}\left|G_{2 r}^{0}\right|^{1-\bar{s} / q} \\
& \leqslant C(\gamma)\left(d^{-\gamma} \int_{G_{2 r}^{0}} u^{\gamma} d x\right)^{\bar{s} / q}\left|G_{2 r}^{0}\right|^{1-\bar{s} / q} \leqslant C(\gamma) d^{-\gamma} \int_{G_{2 r}^{0}} u^{\gamma} d x
\end{aligned}
$$

Из неравенства (8.18) и двух последних соотношений будем иметь

$$
d^{-\gamma} r^{-n} \int_{G_{r}^{0}} u^{\gamma} d x \leqslant C(n, \bar{s}, \gamma)\left(r^{\bar{s}} \int_{B_{2 r}^{x_{0}}}|\nabla w|^{\bar{s}} \eta^{s} d x+d^{-\gamma} r^{-n} \int_{G_{2 r}^{0}} u^{\gamma} d x\right)^{q / \bar{s}}
$$

Для оценки первого интеграла в правой части (8.19) заметим, что

$$
r^{\bar{s}} \int_{B_{2 r}^{x_{0}}}|\nabla w|^{\bar{s}} \eta^{s} d x \leqslant C(\gamma) d^{-\bar{s}} r^{\bar{s}-n} \int_{G_{2 r}^{0}}|\nabla u|^{\bar{s}}\left(1+\frac{u}{d}\right)^{-\tau} \eta^{s} d x
$$


где

$$
\tau=\frac{\gamma \bar{s}}{p_{0}\left(p_{0}-1\right)}, \quad \tau>1, \quad \rho \leqslant \rho_{0}(p, \gamma) .
$$

По неравенству Юнга имеем

$$
\begin{aligned}
|\nabla u|^{\bar{s}}\left(1+\frac{u}{d}\right)^{-\tau} \eta^{s} & \leqslant|\nabla u|^{p(x)}\left(1+\frac{u}{d}\right)^{-\tau} \eta^{s}+\left(1+\frac{u}{d}\right)^{-\tau} \eta^{s} \\
& \leqslant|\nabla u|^{p(x)}\left(1+\frac{u}{d}\right)^{-\tau} \eta^{s}+\left(1+\frac{u}{d}\right)^{\gamma} \eta^{s}
\end{aligned}
$$

Кроме того, $d^{-\bar{s}} \leqslant d^{-p_{0}}$ (см. (8.14)) и $r^{-\bar{s}} \leqslant C(p) r^{-p_{0}}$ в силу логарифмического условия (1.12). В результате, пользуясь еще неравенством (8.12), получим

$$
\begin{aligned}
r^{\bar{s}} \int_{B_{2 r}^{x_{0}}}|\nabla w|^{\bar{s}} \eta^{s} d x \leqslant & C(n, \gamma, p)\left(d^{-p_{0}} r^{p_{0}-n} \int_{G_{2 r}^{0}}|\nabla u|^{p(x)}\left(1+\frac{u}{d}\right)^{-\tau} \eta^{s} d x\right. \\
& \left.+d^{-\gamma} r^{-n} \int_{G_{2 r}^{0}} u^{\gamma} d x\right)
\end{aligned}
$$

Переходя к оценке первого слагаемого в правой части (8.21), выберем в интегральном тождестве (8.1) пробную функцию

$$
\psi(x)=\left(1-\left(1+\frac{u(x)}{d}\right)^{1-\tau}\right) \eta^{s}(x),
$$

где $\eta(x)$ имеет тот же смысл, что и выше. Поскольку $u(x)$ - емкостный потенциал компакта $E_{\rho} \subset B_{8 \rho}^{x_{0}}$, то сушествует последовательность функций $u_{j} \in C_{0}^{\infty}\left(B_{8 \rho}^{x_{0}}\right)$, равных единице на $E_{\rho}$, такая, что $u_{j} \rightarrow u$ в $W_{0}\left(B_{8 \rho}^{x_{0}}\right)$. Соответствующая последовательность функций

$$
\psi_{j}(x)=\left(1-\left(1+\frac{u_{j}(x)}{d}\right)^{1-\tau}\right) \eta^{s}(x)
$$

сходится в $W_{0}\left(B_{8 \rho}^{x_{0}}\right)$ к $\psi(x)$ и состоит из функций, удовлетворяющих неравенству $\psi_{j}(x) \leqslant 2$ при $x \in E_{\rho}$. Поэтому, переходя в интегральном тождестве

$$
\int_{B_{2 r}^{x_{0}}}|\nabla u|^{p(x)-2} \nabla u \cdot \nabla \psi_{j} d x=\int_{B_{2 r}^{x_{0}}} \psi_{j} d \mu
$$

к пределу при $j \rightarrow \infty$, придем к соотношению

$$
\int_{B_{2 r}^{x_{0}}}|\nabla u|^{p(x)-2} \nabla u \cdot \nabla \psi d x \leqslant 2 \mu\left(B_{2 r}^{x_{0}}\right) .
$$

После несложных преобразований, использующих явный вид $\psi(x)$ и неравенство Юнга, будем иметь

$$
\begin{aligned}
& \int_{G_{2 r}^{0}}|\nabla u|^{p(x)}\left(1+\frac{u}{d}\right)^{-\tau} \eta^{s} d x \\
& \quad \leqslant C(n, \gamma, \bar{s})\left(\int_{G_{2 r}^{0}}\left(\frac{d}{r}\right)^{p(x)}\left(1+\frac{u}{d}\right)^{p(x)-\tau} d x+d \cdot \mu\left(B_{2 r}^{x_{0}}\right)\right) .
\end{aligned}
$$


Далее, так как $\tau>1$ (см. (8.20)) и в силу предположения леммы $\gamma>p_{0}-1$, то $p(x)-\tau<\gamma+p(x)-p_{0}$. Поэтому, используя (8.14) и условие (1.12), получим

$$
\left(1+\frac{u(x)}{d}\right)^{p(x)-\tau} \leqslant C(p)\left(1+\frac{u(x)}{d}\right)^{\gamma}, \quad\left(\frac{d}{r}\right)^{p(x)} \leqslant C(p) d^{p_{0}} r^{-p_{0}}, \quad x \in G_{2 r}^{0} .
$$

Теперь с учетом (8.12) неравенство (8.22) примет вид

$$
\int_{G_{2 r}^{0}}|\nabla u|^{p(x)}\left(1+\frac{u}{d}\right)^{-\tau} \eta^{s} d x \leqslant C(n, \gamma, p)\left(d^{p_{0}-\gamma} r^{-p_{0}} \int_{G_{2 r}^{0}} u^{\gamma} d x+d \cdot \mu\left(B_{2 r}^{x_{0}}\right)\right)
$$

и из (8.21), (8.19) получим оценку

$$
\begin{aligned}
& d^{-\gamma} r^{-n} \int_{G_{r}^{0}} u^{\gamma} d x \leqslant \\
& \quad \leqslant C(n, \gamma, p)\left(d^{-\gamma} r^{-n} \int_{G_{2 r}^{0}} u^{\gamma} d x+d^{1-p_{0}} r^{p_{0}-n} \mu\left(B_{2 r}^{x_{0}}\right)\right)^{q / \bar{s}} .
\end{aligned}
$$

Заметим (см. (8.15)), что $\frac{q}{\bar{s}}=\frac{q}{p_{0}}+\frac{q\left(p_{0}-\bar{s}\right)}{\bar{s} p_{0}}$. Поскольку

$$
\mu\left(B_{2 r}^{x_{0}}\right) \leqslant C_{p}\left(\bar{B}_{r}^{x_{0}}, B_{2 r}^{x_{0}}\right) \leqslant C(n, p) r^{n-p_{0}}
$$

(см. (5.4)), то согласно (8.14) и условию (1.12) имеем

$$
\left(d^{-\gamma} r^{-n} \int_{G_{2 r}^{0}} u^{\gamma} d x+d^{1-p_{0}} r^{p_{0}-n} \mu\left(B_{2 r}^{x_{0}}\right)\right)^{q\left(p_{0}-\bar{s}\right) / \bar{s} p_{0}} \leqslant C(n, \gamma, p),
$$

и из (8.23) вытекает требуемое неравенство (8.10). Лемма доказана.

ТЕОРема 8.1. Для любого $\gamma>p_{0}-1$ nри $\rho \leqslant \rho_{0}(n, p, \gamma)$ имеет место неравенство

$$
u\left(x_{0}\right) \leqslant C(n, \gamma, p)\left(f_{B_{\rho}^{x_{0}}} u^{\gamma} d x\right)^{1 / \gamma}+C(n, \gamma, p) \sum_{j=1}^{\infty}\left(\frac{\mu\left(B_{r_{j}}^{x_{0}}\right)}{r_{j}^{n-p_{0}}}+r_{j}^{p_{0}-1}\right)^{1 /\left(p_{0}-1\right)},
$$

в котором $r_{j}=2^{1-j} \rho, j=0,1, \ldots$.

ДоКАЗАТЕЛЬСТВо. Не ограничивая обшности, можно предположить

$$
\gamma<\frac{n\left(p_{0}-1\right)}{n-p_{0}+1}
$$

так как для остальных значений $\gamma$ требуемое утверждение вытекает из неравенства Гёльдера. Зафиксируем константу $\delta \in(0,1)$, которая будет определена позже, и, задав последовательность $\left\{a_{j}\right\}$ :

$$
a_{0}=0, \quad a_{j+1}=a_{j}+\delta^{-1}\left(r_{j}^{-n} \int_{G_{r_{j+1}}^{a_{j}}}\left(u-a_{j}\right)^{\gamma} d x\right)^{1 / \gamma}, \quad j=0,1, \ldots,
$$


в которой множество $G_{r_{j}}^{a_{j}}$ имеет тот же смысл, что и в лемме 8.2 , докажем неравенство

$$
a_{j+1}-a_{j} \leqslant \frac{1}{2}\left(a_{j}-a_{j-1}\right)+C(n, \gamma, p)\left(\frac{\mu\left(B_{r_{j}}^{x_{0}}\right)}{r_{j}^{n-p_{0}}}+r_{j}^{p_{0}-1}\right)^{1 /\left(p_{0}-1\right)} .
$$

Сначала, предполагая

$$
a_{j+1}-a_{j} \geqslant 2^{(n+1) / \gamma} r_{j}
$$

установим оценку

$$
\delta^{p_{0} \gamma / q} \leqslant C(n, \gamma, p) \delta^{\gamma}\left(\frac{a_{j}-a_{j-1}}{a_{j+1}-a_{j}}\right)^{\gamma}+C(n, \gamma, p)\left(a_{j+1}-a_{j}\right)^{1-p_{0}} \frac{\mu\left(B_{r_{j}}^{x_{0}}\right)}{r_{j}^{n-p_{0}}}
$$

где $q$ - постоянная леммы $8.2($ см. (8.15)). Имеем

$$
\begin{aligned}
\left|G_{r_{j}}^{a_{j}}\right| & \leqslant\left(a_{j}-a_{j-1}\right)^{-\gamma} \int_{G_{r_{j}}^{a_{j}}}\left(u-a_{j-1}\right)^{\gamma} d x \\
& \leqslant\left(a_{j}-a_{j-1}\right)^{-\gamma} \int_{G_{r_{j}}^{a_{j-1}}}\left(u-a_{j-1}\right)^{\gamma} d x \\
& =\delta^{\gamma}\left(r_{j-1}^{-n} \int_{G_{r_{j}}^{a_{j-1}}}\left(u-a_{j-1}\right)^{\gamma} d x\right)^{-1} \int_{G_{r_{j}-1}^{a_{j-1}}}\left(u-a_{j-1}\right)^{\gamma} d x \\
& =\delta^{\gamma} r_{j-1}^{n}=\delta^{\gamma} 2^{n} r_{j}^{n}=2^{n}\left(a_{j+1}-a_{j}\right)^{-\gamma} \int_{G_{r_{j+1}}^{a_{j}}}\left(u-a_{j}\right)^{\gamma} d x .
\end{aligned}
$$

Постоянную $\delta>0$ подчиним ограничению $\delta^{\gamma} \leqslant 2^{-n-1} r_{j}^{-n}\left|B_{r_{j}}^{x_{0}}\right|$, влекущему двойное неравенство

$$
\left|G_{r_{j}}^{a_{j}}\right| \leqslant \delta^{\gamma} 2^{n} r_{j}^{n} \leqslant \frac{1}{2}\left|B_{r_{j}}^{x_{0}}\right|
$$

Из соотношений (8.28) и (8.26) следует, что условие (8.9) выполнено с постоянной

$$
d_{j}=2^{-(n+1) / \gamma}\left(a_{j+1}-a_{j}\right) \geqslant r_{j}
$$

и в силу оценки (8.10) имеем

$$
\begin{aligned}
& \left(d_{j}^{-\gamma} r_{j}^{-n} \int_{G_{r_{j}+1}^{a_{j}}}\left(u-a_{j}\right)^{\gamma} d x\right)^{p_{0} / q} \\
& \quad \leqslant C(n, \gamma, p)\left(d_{j}^{-\gamma} r_{j}^{-n} \int_{G_{r_{j}}^{a_{j}}}\left(u-a_{j}\right)^{\gamma} d x+d_{j}^{1-p_{0}} r_{j}^{p_{0}-n} \mu\left(B_{r_{j}}^{x_{0}}\right)\right) .
\end{aligned}
$$


Далее, так как

$$
\begin{gathered}
d_{j}^{-\gamma} r_{j}^{-n} \int_{G_{r_{j}}^{a_{j}}}\left(u-a_{j}\right)^{\gamma} d x \leqslant d_{j}^{-\gamma} r_{j}^{-n} \int_{G_{r_{j}}^{a_{j-1}}}\left(u-a_{j-1}\right)^{\gamma} d x \\
=d_{j}^{-\gamma} \delta^{\gamma}\left(a_{j}-a_{j-1}\right)^{\gamma}=C(n)\left(\frac{d_{j-1}}{d_{j}}\right)^{\gamma}, \\
\delta^{p_{0} \gamma / q}=C(n, \gamma)\left(d_{j}^{-\gamma} r_{j}^{-n} \int_{G_{r_{j+1}}^{a_{j}}}\left(u-a_{j}\right)^{\gamma} d x\right)^{p_{0} / q},
\end{gathered}
$$

то с учетом (8.31) получим

$$
\begin{aligned}
\delta^{p_{0} \gamma / q} & \leqslant C(n, \gamma, p)\left(d_{j}^{-\gamma} r_{j}^{-n} \int_{G_{r_{j}}^{a_{j}}}\left(u-a_{j}\right)^{\gamma} d x+d_{j}^{1-p_{0}} r_{j}^{p_{0}-n} \mu\left(B_{r_{j}}^{x_{0}}\right)\right) \\
& \leqslant C(n, \gamma, p) \delta^{\gamma}\left(\frac{a_{j}-a_{j-1}}{a_{j+1}-a_{j}}\right)^{\gamma}+C(n, \gamma, p)\left(a_{j+1}-a_{j}\right)^{1-p_{0}} \frac{\mu\left(B_{r_{j}}^{x_{0}}\right)}{r_{j}^{n-p_{0}}},
\end{aligned}
$$

что и доказывает (8.27).

Перейдем к выводу (8.25). Если $a_{j+1}-a_{j} \leqslant \frac{1}{2}\left(a_{j}-a_{j-1}\right)$ или $d_{j}<r_{j}$ (см. (8.30)), то требуемое неравенство очевидно. Если же $a_{j}-a_{j-1}<2\left(a_{j+1}-a_{j}\right)$ и $d_{j} \geqslant r_{j}$, то из (8.27) следует, что

$$
\delta^{p_{0} \gamma / q} \leqslant C(n, \gamma, p) \delta^{\gamma}+C(n, \gamma, p)\left(a_{j+1}-a_{j}\right)^{1-p_{0}} \frac{\mu\left(B_{r_{j}}^{x_{0}}\right)}{r_{j}^{n-p_{0}}}
$$

Выбирая $\delta=\delta(n, \gamma, p)$ из условия $\delta^{p_{0} \gamma / q}>2 C(n, \gamma, p) \delta^{\gamma}$, где $C$ - постоянная из предыдушего соотношения, будем иметь

$$
a_{j+1}-a_{j} \leqslant\left(\frac{\mu\left(B_{r_{j}}^{x_{0}}\right)}{r_{j}^{n-p_{0}}}\right)^{1 /\left(p_{0}-1\right)}
$$

т. е. неравенство (8.25) выполнено и в этом случае.

Из (8.25) вытекает оценка

$$
\begin{aligned}
a_{k}-a_{1} & \leqslant a_{k+1}-a_{1}=\sum_{j=1}^{k}\left(a_{j+1}-a_{j}\right) \\
& \leqslant \frac{1}{2} \sum_{j=1}^{k}\left(a_{j}-a_{j-1}\right)+C(n, p, \gamma) \sum_{j=1}^{k}\left(\frac{\mu\left(B_{r_{j}}^{x_{0}}\right)}{r_{j}^{n-p_{0}}+r_{j}^{p_{0}-1}}\right)^{1 /\left(p_{0}-1\right)} \\
& =\frac{1}{2} a_{k}+C(n, p, \gamma) \sum_{j=1}^{k}\left(\frac{\mu\left(B_{r_{j}}^{x_{0}}\right)}{r_{j}^{n-p_{0}}}+r_{j}^{p_{0}-1}\right)^{1 /\left(p_{0}-1\right)}
\end{aligned}
$$


в силу которой

$$
\begin{aligned}
\lim _{k \rightarrow \infty} a_{k} & \leqslant 2 a_{1}+C(n, \gamma, p) \sum_{j=1}^{\infty}\left(\frac{\mu\left(B_{r_{j}}^{x_{0}}\right)}{r_{j}^{n-p_{0}}}+r_{j}^{p_{0}-1}\right)^{1 /\left(p_{0}-1\right)} \\
& \leqslant C(n, \gamma, p)\left(f_{B_{\rho}} u^{\gamma} d x\right)^{1 / \gamma}+C(n, \gamma, p) \sum_{j=1}^{\infty}\left(\frac{\mu\left(B_{r_{j}}^{x_{0}}\right)}{r_{j}^{n-p_{0}}}+r_{j}^{p_{0}-1}\right)^{1 /\left(p_{0}-1\right)}
\end{aligned}
$$

Согласно (8.29) $\inf _{B_{r_{j}}} u \leqslant a_{j}$ для $j=1,2, \ldots$, а в силу полунепрерывности снизу емкостного потенщиала имеем

$$
u\left(x_{0}\right)=\lim _{j \rightarrow \infty} \inf _{B_{r_{j}}} u \leqslant \lim _{j \rightarrow \infty} a_{j}
$$

Сопоставляя (8.32) и (8.33), придем к требуемому неравенству (8.24). Теорема доказана.

Выберем в (8.18) постоянную $\gamma>p_{0}-1$, удовлетворяюшую соотношению $\gamma<n\left(p_{0}-1\right) /(n-1)$. По неравенству Харнака слабого типа для неотрицательных суперрешений (см. лемму 6.4) имеем

$$
\left(f_{B_{\rho}^{x_{0}}} u^{\gamma} d x\right)^{1 / \gamma} \leqslant C(n, p)\left(f_{B_{4 \rho}^{x_{0}}} u^{\gamma} d x\right)^{1 / \gamma} \leqslant C(n, p)\left(\inf _{B_{2 \rho}^{x_{0}}} u(x)+\rho\right)
$$

и (8.18) примет вид

$$
u\left(x_{0}\right) \leqslant C(n, p) \inf _{B_{2 \rho}^{x_{0}}} u(x)+C(n, p) \sum_{j=1}^{\infty}\left(\frac{\mu\left(B_{r_{j}}^{x_{0}}\right)}{r_{j}^{n-p_{0}}}+r_{j}^{p_{0}-1}\right)^{1 /\left(p_{0}-1\right)}
$$

Следуюшее утверждение посвяшено оценке первого слагаемого в правой части (8.34). Напомним, что $u(x)$ - емкостной потенщиал компакта $E_{\rho}=\left(\mathbb{R}^{n} \backslash D\right) \cap \bar{B}_{\rho}^{x_{0}}$ относительно шара $B_{8 \rho}^{x_{0}}$.

ЛЕмма 8.3. Справедливо неравенство

$$
\inf _{B_{2 \rho}^{x_{0}}} u(x) \leqslant C(p)\left(\frac{\mu\left(B_{\rho}^{x_{0}}\right)}{\rho^{n-p_{0}}}+\rho^{p_{0}-1}\right)^{1 /\left(p_{0}-1\right)}
$$

ДокАЗАТЕЛЬСтво. Положим

$$
\lambda=\min _{B_{2 \rho}^{x_{0}}} u(x), \quad \varphi(x)=\min (u(x), \lambda)
$$

и, не ограничивая общности, будем считать $\lambda>0$. Ясно, что $\varphi \in W_{0}\left(B_{8 \rho}^{x_{0}}\right)$ и $\varphi(x)=\lambda$ при $x \in \bar{B}_{2 \rho}^{x_{0}}$. Пусть $\left\{u_{j}\right\} \in C_{0}^{\infty}\left(B_{8 \rho}^{x_{0}}\right)$ - последовательность, состоящая из функций, равных единице в окрестности компакта $E_{\rho}$, и сходящаяся в $W_{0}\left(B_{8 \rho}^{x_{0}}\right)$ 
к емкостному потенциалу $u(x)$. Существование такой последовательности установлено в доказательстве теоремы 5.1. Отсюда и из свойств б)-г) леммы 2.5 следует существование последовательности $\left\{v_{j}\right\} \in C_{0}^{\infty}\left(B_{8 \rho}^{x_{0}}\right)$ такой, что $v_{j}(x)=\lambda$ в окрестности $B_{\rho}^{x_{0}}$ и $v_{j}(x) \rightarrow \varphi(x)$ в $W_{0}\left(B_{8 \rho}^{x_{0}}\right)$. Выбирая в интегральном тождестве (8.1) пробную функцию $\psi(x)=v_{j}(x)$, после перехода к пределу будем иметь

$$
\lim _{j \rightarrow \infty} \int_{B_{8 \rho}^{x_{0}}}\left|\nabla v_{j}\right|^{p(x)} d x=\int_{B_{8 \rho}^{x_{0}}}|\nabla \varphi|^{p(x)} d x \leqslant \lambda \mu\left(B_{\rho}^{x_{0}}\right) .
$$

С другой стороны, поскольку $0<\lambda \leqslant 1$, то

$$
\lambda^{l} C_{p}\left(\bar{B}_{\rho}^{x_{0}}, B_{8 \rho}^{x_{0}}\right) \leqslant \int_{B_{8 \rho}^{x_{0}}} \frac{\lambda^{p(x)}}{p(x)}\left|\frac{\nabla v_{j}}{\lambda}\right|^{p(x)} d x \leqslant C(p) \int_{B_{8 \rho}^{x_{0}}}\left|\nabla v_{j}\right|^{p(x)} d x,
$$

где $l=\sup _{B_{8 \rho}^{x_{0}}} p(x)$. Так как $C_{p}\left(\bar{B}_{\rho}^{x_{0}}, B_{8 \rho}^{x_{0}}\right) \geqslant C(n, p) \rho^{n-p_{0}}$ (см. (5.5)), то требуемое неравенство (8.35) является простым следствием (8.36), (8.37) и условия (1.12). Лемма доказана.

2. Доказательство необходимого условия регулярности граничной точки. Покажем, что сходимость в нуле интеграла (1.19) влечет иррегулярность граничной точки $x_{0} \in \partial D$. Для этого, основываясь на утверждении леммы 8.1, покажем существование достаточно малого $\rho$, для которого емкостный потенциал $u(x)=u_{\rho}(x)$ компакта $E_{\rho}$ относительно шара $B_{8 \rho}^{x_{0}}$ удовлетворяет условию (8.2).

Пусть $E=\mathbb{R}^{n} \backslash D$ и

$$
\gamma_{j}=C_{p}\left(E \cap \bar{B}_{2^{1-j}}^{x_{0}}, B_{2^{-j}}^{x_{0}}\right) 2^{(1-j)\left(p_{0}-n\right)} .
$$

Сходимость в нуле интеграла (1.19) эквивалентна сходимости ряда

$$
\sum_{j=1}^{n}\left(\gamma_{j}\right)^{\frac{1}{p_{0}-1}}<\infty
$$

В силу неравенств (8.34), (8.35) имеем

$$
u\left(x_{0}\right) \leqslant C(n, p) \sum_{j=1}^{\infty}\left(\frac{\mu\left(B_{r_{j}}^{x_{0}}\right)}{r_{j}^{n-p_{0}}}+r_{j}^{p_{0}-1}\right)^{\frac{1}{p_{0}-1}}
$$

где $r_{j}=2^{1-j} \rho, j=1,2, \ldots$ Выбирая здесь $\rho=2^{-k}$ и пользуясь оценкой (5.16), согласно которой $\mu\left(B_{j}^{x_{0}}\right) \leqslant C(p) C_{p}\left(E \cap \bar{B}_{r_{j}}^{x_{0}}, B_{r_{j-1}}^{x_{0}}\right)$, будем иметь

$$
u\left(x_{0}\right) \leqslant C(n, p) \sum_{j=1}^{\infty}\left(\gamma_{j+k}+2^{(1-j-k)\left(p_{0}-1\right)}\right)^{\frac{1}{p_{0}-1}} .
$$

Теперь из сходимости ряда (8.38) следует сушествование номера $k=k(n, p)$ такого, что $u\left(x_{0}\right)<1$. Необходимость доказана. 
3. Геометрические условия регулярности. Пусть в окрестности граничной точки, совпадаюшей с началом координат, внутренность дополнения области $D$ совпадает с областью вида (1.22). Полагая $x^{\prime}=\left(x_{1}, x_{2}, \ldots, x_{n-1}\right)$ и $x=$ $\left(x^{\prime}, x_{n}\right)$, доопределим показатель суммируемости $p(x)$, считая, что $p\left(0, x_{n}\right)=p_{0}=$ $p(0)$ при $x_{n} \in[0, a / 2]$, где $a-$ постоянная из (1.22). В силу наложенных ограничений на функцию $g(t)$, с помошью которой задается область (1.22), доопределенный таким образом показатель $p(x)$ удовлетворяет на множестве $\bar{D} \cup\left\{\left(x^{\prime}, x_{n}\right): x^{\prime}=0\right.$, $\left.x_{n} \in[0, a / 2]\right\}$ условиям (1.2) и (1.12). Продолжим теперь $p(x)$ на все $\mathbb{R}^{n}$ с сохранением этих же условий. Ниже $B_{R}$ означает шар с центром в начале координат.

ДокаЗАТЕЛЬСтво теОремы 1.4. Достаточность. Оценим снизу емкость компакта $E_{R}=\left(\mathbb{R}^{n} \backslash D\right) \cap \bar{B}_{R}$ относительно шара $B_{2 R}$. Пусть $\varphi \in C_{0}^{\infty}\left(B_{2 R}\right)$ и $\varphi=1$ в окрестности $E_{R}$. Ясно, что

$$
\int_{B_{2 R}}|\nabla \varphi|^{p(x)} d x \geqslant \int_{R / 8}^{R / 4} d x_{n} \int_{Q_{2 R}}\left|\nabla^{\prime} \varphi\right|^{p(x)} d x^{\prime},
$$

где $\left|\nabla^{\prime} \varphi\right|=\left(\sum_{i=1}^{n-1} \varphi_{x_{i}}^{2}\right)^{1 / 2}$, а $Q_{r}-(n-1)$-мерный шар радиуса $r$ с центром в начале координат. Для каждого фиксированного $R / 8 \leqslant x_{n} \leqslant R / 4$ имеем

$$
\int_{Q_{2 R}}\left|\nabla^{\prime} \varphi\right|^{p(x)} d x^{\prime} \geqslant C(p) C_{p}\left(\bar{Q}_{r}, Q_{2 R}\right), \quad r=g\left(x_{n}\right) .
$$

Минимизируя интеграл в левой части (8.39) и пользуясь нижними оценками (5.5) емкости $(n-1)$-мерных шаров, получим

$$
C_{p}\left(E_{R}, B_{2 R}\right) \geqslant \begin{cases}C \int_{R / 8}^{R / 4}(g(t))^{n-1-p_{0}} d t, & p_{0}<n-1, \\ C \int_{R / 8}^{R / 4}\left(\ln \frac{2 R}{g(t)}\right)^{2-n} d t, & p_{0}=n-1, \\ C R^{n-p_{0}}, & n-1<p_{0} \leqslant n .\end{cases}
$$

Поскольку функция $g(t)$ не убывает, то для $\gamma(R)=C_{p}\left(E_{R}, B_{2 R}\right) R^{p_{0}-n}$ справедливы оценки

$$
\gamma(R) \geqslant \begin{cases}C\left(\frac{g(R / 8)}{R}\right)^{n-1-p_{0}}, & p_{0}<n-1, \\ C\left(\ln \frac{2 R}{g(R / 8)}\right)^{2-n}, & p_{0}=n-1, \\ C, & n-1<p_{0} \leqslant n .\end{cases}
$$

Отсюда, пользуясь при $p_{0}=n-1$ условием $g(t) \geqslant t^{\alpha}$, найдем, что

$$
\int_{r}^{\rho}(\gamma(t))^{1 /\left(p_{0}-1\right)} t^{-1} d t \geqslant \begin{cases}C \int_{r}^{\rho}\left(\frac{g(t / 8)}{t}\right)^{\frac{n-1-p_{0}}{p_{0}-1}} t^{-1} d t, & p_{0}<n-1, \\ C \int_{r}^{\rho}\left(\ln \frac{1}{t}\right)^{-1} t^{-1} d t, & p_{0}=n-1, \\ C \ln \frac{\rho}{r}, & n-1<p_{0} \leqslant n .\end{cases}
$$


Таким образом, условие (1.19) для описанной выше области в случае $p_{0}<n-1$ выполнено, если расходится интеграл (1.23). Если же $p_{0} \geqslant n-1$, то, как показывает простой подсчет, граничная точка регулярна.

Необходимость. Покажем, что если $p_{0}<n-1$, то условие (1.23) необходимо для регулярности начала координат. Для этого оценим сверху емкость цилиндра $\Pi_{r, R}=\left\{x=\left(x^{\prime}, x_{n}\right):\left|x^{\prime}\right| \leqslant r,\left|x_{n}\right| \leqslant R\right\}, r \leqslant R$, относительно шара $B_{2 R}$. Пусть $\varphi\left(x^{\prime}\right)$ - емкостный потенциал $(n-1)$-мерного шара $\bar{Q}_{r}$ с центром в начале координат относительно конщентрического шара $Q_{2}$. Введем еше финитную бесконечно дифференцируемую на отрезке $[-3 R / 2,3 R / 2]$ функцию $\eta\left(x_{n}\right)$, удовлетворяющую условиям: $0 \leqslant \eta \leqslant 1, \eta\left(x_{n}\right)=1$ при $x_{n} \in[-R, R]$ и $\left|\eta^{\prime}\right| \leqslant C R^{-1}$. Как нетрудно видеть,

$$
\begin{aligned}
C_{p}\left(\Pi_{r, R}, B_{2 R}\right) & \leqslant C(p) \int_{B_{2 R}}|\nabla(\varphi \eta)|^{p(x)} d x \\
& \leqslant C(p)\left(\int_{B_{2 R}}|\nabla \varphi|^{p(x)} \eta^{p(x)} d x+\int_{B_{2 R}}|\varphi|^{p(x)}|\nabla \eta|^{p(x)} d x\right) .
\end{aligned}
$$

Пользуясь логарифмическим условием (1.12), неравенством $0 \leqslant \varphi \leqslant 1$, верхними оценками емкости (5.4) и условием (1.12), получим

$$
C_{p}\left(\Pi_{r, R}, B_{2 R}\right) \leqslant C(n, p)\left(R r^{n-p_{0}-1}+R^{1-p_{0}} r^{n-1}\right) \leqslant C(n, p) R r^{n-p_{0}-1} .
$$

Поскольку $E_{R} \subset \Pi_{r, R}$ при $r=g(R)$, то согласно предыдушей оценке имеем

$$
\int_{r}^{\rho}(\gamma(t))^{1 /\left(p_{0}-1\right)} t^{-1} d t \leqslant C(n, p) \int_{r}^{\rho}\left(\frac{g(t)}{t}\right)^{\frac{n-1-p_{0}}{p_{0}-1}} t^{-1} d t .
$$

Отсюда следует, что условие (1.23) необходимо для регулярности граничной точки. Теорема доказана.

\section{Список литературы}

1. Ладыженская О.А., Уральцева H. Н. Линейные и квазилинейные уравнения эллиптического типа. М.: Наука, 1964.

2. Жиков В. В. Вопросы сходимости, двойственности и усреднения для функционалов вариационного исчисления // Изв. АН СССР. Сер. матем. 1983. Т. 47. № 5. С. 961-995.

3. Жиков В. В. О постановке краевых задач для интегралов вида $|\xi|^{\alpha(x)} / /$ УМН. 1986. T. 41. № 4. C. 187-188.

4. Жиков В. В. Усреднение функционалов вариационного исчисления и теории упругости // Изв. АН СССР. Сер. матем. 1986. Т. 50. № 4. С. 675-711.

5. Giaquinta M. Growth Conditions and Regularity, a Counterexample // Manuscripta Math. 1987. V. 59. P. 245-248.

6. Marcellini P. Un Exemple de Solution Discontinue d'un Probléme Variationell dans le cas scalaire. Preprint № 11 / Instituto Matematico U. Dini. Universitá di Firenze, 1987.

7. Rüzhǐcka M. Electrorheological Fluids: Modeling and Mathematical Theory // Lecture Notes in Math. 2000. V. 1748.

8. Zhikov V. V. On some variational problems // Russian J. of Math. Physics. 1996. V. 5. № 1. P. 105-116. 
9. Lavrentieff $M$. Sur quelques problémes du calcul des variations // Ann. Mat. Pura Appl. 1926. V. 4. P. 7-28.

10. Zhikov V. V. On Lavrentiev's Phenomenon // Russian J. of Math. Physics. 1994. V. 3. № 2. P. 249-269.

11. Zhikov V. V. Meyers type estimates for the solution of a nonlinear Stokes system // Diff. Eq. 1997. V. 33. № 1. P. 108-115.

12. Leray J., Lions $J$.-L. Quelques résultats de Višik sur les problémes elliptiques non linéaires par les méthodes de Minty-Brouder // Bull. Soc. Math. France. 1965. V. 93. P. 97-107.

13. Ладыженская $O . A .$, Уральцева $H . H$. Квазилинейные эллиптические уравнения и вариационные задачи со многими независимыми переменными // УМН. 1961. Т. 16. № 1. C. $19-90$.

14. Marcellini P. Regularity for Elliptic Equations with General Growth Conditions // J. Diff. Equations. 1993. V. 105. P. 296-333.

15. Fan X. A Class of De Giorgi Type and Holder Continuity of Minimizers of Variational with $m(x)$-Growth Condition. China: Lanzhou Univ., 1995.

16. Алхутов Ю.А. Неравенство Харнака и гёльдеровость решений нелинейных эллиптических уравнений с нестандартным условием роста // Дифф. уравнения. 1997. Т. 33. № 12. C. $1651-1660$.

17. Chiado Piat V., Coscia A. Hölder continuity of minimiizers of functionals with variable growth exponent // Manuscripta Math. 1997. V. 93. P. 283-299.

18. Acerbi E., Fusco N.A. Transmission problem in the calculus of variation // Calc. Var. 1994. V. 2. P. 1-16.

19. Крашенинникова О.В. О непрерывности в точке решений эллиптических уравнений с нестандартным условием роста // Тр. Матем. ин-та им. В.А. Стеклова РАН. 2002. T. 236. C. 204-211.

20. Кондратьев В.А., Ландис E. М. Качественная теория линейных дифференциальных уравнений в частных производных второго порядка // Итоги науки и техники. Совр. проблемы математики. Фундаментальные направления. Т. 32. М.: ВИНИТИ, 1988. C. $99-215$.

21. Heinonen J., Kilpeläinen T., Martio O. Nonlinear Potential Theory of Degenerate Elliptic Equations. Oxford: Clarendon Press, 1993.

22. Wiener N. Certain notions in potential theory // J. Math. Phys. 1924. V. 3. P. 24-51.

23. Littman W., Stampacchia G., Weinberger H. F. Regular points for elliptic equations with discontinuous coefficients // Ann. Scuola Norm. Sup. Pisa. 1963. V. 17. № 3. P. 43-77.

24. Мазья В. Г. О непрерывности в граничной точке решений квазилинейных эллиптических уравнений // Вестн. ЛГУ. Сер. матем. 1970. Т. 25. № 13. С. 42-55.

25. Gariepy R., Ziemer W.P. A regularity condition at the boundary for solutions of quasilinear elliptic equations // Arch. Rational Mech. Anal. 1977. V. 67. P. 25-39.

26. Скрыпник И. В. Критерий регулярности граничной точки для квазилинейных эллиптических уравнений // ДАН СССР. 1984. Т. 274. № 5. С. 1040-1050.

27. Kilpeläinen T., Malý J. The Wiener test and potential estimates for quasilinear elliptic equations // Acta Math. 1994. V. 172. P. 137-161.

28. Lindkvist P., Martio $O$. Two theorems of N. Wiener for solutions of quasilinear elliptic equations // Acta Math. 1985. V. 155. P. 153-171.

29. Choquet G. Theory of capacities // Annales de l'Institut Fourier. 1953-1954. V. 155. № 5. P. 131-295.

30. Кондратьев B. A. О разрешимости первой краевой задачи для сильно эллиптических уравнений // Тр. ММО. 1967. Т. 16. С. 209-292.

31. Экланд И., Темам Р. Выпуклый анализ и вариационные проблемы. М.: Мир, 1979.

32. Trudinger N.S. On Harnack type inequalities and their application to quasilinear elliptic equations // Comm. Pure Appl. Math. 1967. V. 20. P. 721-747.

33. Stampacchia $G$. Le probléme de Dirichlet pour les équations elliptiques du second ordre á coefficients discontinuous // Ann. Inst. Fourier. 1965. V. 15. P. 189-258. 
34. Lindkvist $P$. On the equation $\operatorname{div}\left(|\nabla u|^{p-2} \nabla u\right)+\lambda|u|^{p-2} u=0 / /$ Proc. Amer. Math. Soc. 1990. V. 109. P. 157-164.

35. Gehring F. W. The $L^{p}$-integrability of the partial derivatives of a quasiconformal mapping // Acta Math. 1973. V. 130. P. 265-277.

36. Giaquinta M., Modica G. Regularity results for some classes of higher order nonlinear elliptic systems // J. für die reine und angewandte Math. 1979. V. 311/312. P. 145-169.

37. Schwartz L. Théorie des Distributions. V. 1. Paris: Hermann, 1950.

38. John G., Nirenberg L. On functions of bounded mean oscillation // Comm. Pure Appl. Math. 1961. V. 14. P. 415-426.

39. Fabes E. B., Stroock D. W. The $L^{p}$-integrability of Green's functions and fundamental solutions for elliptic and parabolic equations // Duke Math. J. 1984. V. 51. № 4. P. 99-1016.

40. Мазья В. Г. Пространства Соболева. Л.: Изд-во ЛГУ, 1985.

Поступило в редакцию 25.02 .2004 